\title{
Chemical Gas Sensors: Recent Developments, Challenges, and the Potential of Machine Learning-A Review
}

\author{
Usman Yaqoob (D) and Mohammad I. Younis * \\ Department of Physical Science and Engineering, King Abdullah University of Science and Technology, \\ Thuwal 23955-6900, Saudi Arabia; usman.yaqoob@kaust.edu.sa \\ * Correspondence: Mohammad.Younis@kaust.edu.sa
}

Citation: Yaqoob, U.; Younis, M.I.

Chemical Gas Sensors: Recent

Developments, Challenges, and the Potential of Machine Learning-A

Review. Sensors 2021, 21, 2877.

https://doi.org/10.3390/s21082877

Academic Editors: Laura Pigani and Rosalba Calvini

Received: 29 March 2021

Accepted: 15 April 2021

Published: 20 April 2021

Publisher's Note: MDPI stays neutral with regard to jurisdictional claims in published maps and institutional affiliations.

Copyright: (c) 2021 by the authors. Licensee MDPI, Basel, Switzerland. This article is an open access article distributed under the terms and conditions of the Creative Commons Attribution (CC BY) license (https:// creativecommons.org/licenses/by/ $4.0 /)$.

\begin{abstract}
Nowadays, there is increasing interest in fast, accurate, and highly sensitive smart gas sensors with excellent selectivity boosted by the high demand for environmental safety and healthcare applications. Significant research has been conducted to develop sensors based on novel highly sensitive and selective materials. Computational and experimental studies have been explored in order to identify the key factors in providing the maximum active location for gas molecule adsorption including bandgap tuning through nanostructures, metal/metal oxide catalytic reactions, and nano junction formations. However, there are still great challenges, specifically in terms of selectivity, which raises the need for combining interdisciplinary fields to build smarter and high-performance gas/chemical sensing devices. This review discusses current major gas sensing performance-enhancing methods, their advantages, and limitations, especially in terms of selectivity and long-term stability. The discussion then establishes a case for the use of smart machine learning techniques, which offer effective data processing approaches, for the development of highly selective smart gas sensors. We highlight the effectiveness of static, dynamic, and frequency domain feature extraction techniques. Additionally, cross-validation methods are also covered; in particular, the manipulation of the k-fold cross-validation is discussed to accurately train a model according to the available datasets. We summarize different chemresistive and FET gas sensors and highlight their shortcomings, and then propose the potential of machine learning as a possible and feasible option. The review concludes that machine learning can be very promising in terms of building the future generation of smart, sensitive, and selective sensors.
\end{abstract}

Keywords: chemiresistive and FET sensors; carbon materials; 2D TMDCs; metal/metal oxide; density function theory (DFT); selectivity; drift compensation; machine learning; data processing; feature extraction; smart sensors; smart breath analyzers; PCA; classifiers

\section{Introduction}

Today, the rapid expansion in automobiles and industries emerge as a serious threat to the environment and human health safety. Therefore, along with the strict implementation of industrial waste regulations, the demand for highly reliable and accurate sensors has become essential for human survival [1-5]. Over the past decades, several gas-sensing mechanisms have been reported, including resistive/chemiresistive [6], electrochemical [7,8] (amperometric and potentiometric), work function (Schottky diode, metaloxide-semiconductor field-effect transistors (MOSFET) [9,10], etc.), optical (surface plasmon resonance (SPR)), and surface acoustic [11-14]. Among them, resistive type gas sensors have been extensively studied due to their small size, low power consumption, cheap and simple fabrication process [10]. To date, various materials have been developed and used for resistive-type sensing, such as carbon materials (graphene/reduced graphene oxide (rGO) [15-18], carbon nanotubes (CNTs) [9,19], carbon and graphene dots [20-22], three-dimensional (D) graphene [23,24], etc.), 2D transition metal dichalcogenides (TMDCs) [25,26], metal oxides (zinc oxide $(\mathrm{ZnO})$ [27], tin oxide $\left(\mathrm{SnO}_{2}\right)$ [28], tita- 
nium oxide $\left(\mathrm{TiO}_{2}\right)$ [29], tungsten oxide $\left(\mathrm{WO}_{3}\right)$ [30], indium oxide $\left(\mathrm{In}_{2} \mathrm{O}_{3}\right)$ [31,32], nickel oxide $(\mathrm{NiO})[33]$, iron oxide $\left(\mathrm{Fe}_{2} \mathrm{O}_{3}\right)$ [34-36], etc.) [6,37,38], and noble metal catalysts (pallidum $(\mathrm{Pd})$, platinum $(\mathrm{Pt})$, gold $(\mathrm{Au})$, silver $(\mathrm{Ag})$, aluminum $(\mathrm{Al})$, rhodium $(\mathrm{Rh})$, etc.) [15,39-41].

Carbon materials possess a higher surface area and have the capability for trace-level molecule detection at room temperature (RT). However, they are less selective and demonstrate a lower recovery rate due to their high binding energy with the gas molecules [42,43]. On the other hand, metal oxides (MOx) are good candidates to detect a wide range of gas molecules at higher concentration levels with a relatively faster recovery rate. However, they require higher operating temperature $(\mathrm{OT})$ to generate favorable oxygen adsorbents $\left(\mathrm{O}_{2}{ }^{-}, \mathrm{O}^{-}\right.$, and $\left.\mathrm{O}^{2-}\right)$ on sensing surfaces [44-46].

For environmental safety and better monitoring of human health, there is an urgent demand for the development of a sensor with trace-level molecule detection, minimum drift, high sensitivity, fast response/recovery, and excellent selectivity under different environments (dry and humid). With the aim to build a sensor having such properties, researchers have focused on synthesizing novel and sensitive sensing materials (SMs) using different techniques including surface morphology change/modification $[22,27,30,46]$, doping [47], composition/hybridization [15,26,30,44,48,49], p-n junction formation [50,51], and core-shell structures [52-55]. SMs synthesized via these methods certainly have a key impact in improving sensor performances. For instance, recently Wu et al. [39] reported a high-performance $\mathrm{NO}_{2}$ sensor at $\mathrm{RT}$ using boron (B)- and nitrogen (N)-doped 3D reduced graphene oxide hydrogel (RGOH). In comparison with pure RGOH sensors, the B- and $\mathrm{N}$-doped RGOH sensors exhibited 38.9 and 18 times higher responses toward $800 \mathrm{ppb}$ $\mathrm{NO}_{2}$, respectively. Additionally, the fabricated sensors showed good linearity, reversibility, fast response/recovery, and impressive selectivity. The higher sensing performances from B- and N-doped RGOH sensors at RT was ascribed to several factors, including the doping effects of $\mathrm{B}$ and $\mathrm{N}, 3 \mathrm{D}$ porous $\mathrm{rGO}$ architecture with the enlarged surfaceto-volume ratio, pore filling, charge hopping, abundant disorder, oxygenated groups, and high electron mobility [39]. On the contrary, metal oxide gas sensors, which require higher operating temperature, can also show good sensing performance via metal catalyst doping and morphological modification. Recently, Sanger et al. [56] presented a highly sensitive transparent $\mathrm{NO}_{2}$ sensor using aluminum (Al)-doped $\mathrm{ZnO}(\mathrm{AZO})$ hollow nanofiber synthesized via the sputtering method. Their sensors displayed maximum sensitivity at an OT of $250{ }^{\circ} \mathrm{C}$ with a detection range (DR) from 0.5 to $10 \mathrm{ppm}$. The high sensitivity of the transparent sensors was attributed to the higher surface area of the hollow nanofibers and the high impact frequency of the trapped $\mathrm{NO}_{2}$ gas inside the hollow compared to the solid counterpart nanofibers [56]. Similarly, Li et al. [57] reported Pd-Au nanoparticles (NPs) decorated on $\mathrm{SnO}_{2}$ nanosheets (NShs) for formaldehyde and acetone detection. They demonstrated the temperature-dependent selectivity of fabricated sensors toward formaldehyde and acetone. The results determined effective detection of acetone (@250 $\left.{ }^{\circ} \mathrm{C}\right)$ and formaldehyde (@110 ${ }^{\circ} \mathrm{C}$ ) with responses of 6.6 (acetone) and 4.1 (formaldehyde) towards 2 ppm concentration, and their corresponding detection limits were noted as $45 \mathrm{ppb}$ and $30 \mathrm{ppb}$, respectively. The enhanced response was attributed to the chemical sensitization of $\mathrm{Au}$, the electronic sensitization of $\mathrm{Pd}$, and the synergistic effect of $\mathrm{Pd}-\mathrm{Au}$ bimetallic NPs [57].

Additionally, bimetals/bimetal oxide core-shell structures were also studied to obtain better sensing outcomes [40,41]. Most recently, Xu et al. [58] studied formaldehyde ( $\mathrm{HCHO}$ ) detection using bimetal Ag@Pt core-shell nanostructures (NSs) decorated on $\mathrm{ZnO}$ nanowires (NWs) by an inkjet printing method. Optimized (with $\mathrm{Pt}_{60}$ and $\mathrm{Ag}_{40}$ atomic ratio) sensors demonstrated maximum response on an OT of $280^{\circ} \mathrm{C}$ with DR varying from $120 \mathrm{ppb}$ to $2 \mathrm{ppm}$. They described how Ag@Pt core-shell NPs play a vital role as a catalyst during the $\mathrm{HCHO}$ detection process by dramatically enhancing the oxidation of $\mathrm{HCHO}$ molecules on the $\mathrm{ZnO}(\mathrm{NWs})$ surface. As a result, more electron release brings a higher $\mathrm{HCHO}$ sensing response for the $\mathrm{ZnO}$-based gas sensor [58]. 
Tuning the material properties through the mentioned techniques significantly improves almost all aspects of the sensor's performance. However, realizing a sensor with excellent selectivity under humid conditions, trace level molecule detection, and good repeatability with no drift error due to aging and environment (temperature and humidity) is still a pressing challenge for the ongoing massive research in the field.

It is well known that SMs tend to lose their properties with aging and environmental factors, and thus can show degradation in response and sensitivity towards unwanted gases. Therefore, along with the development of highly stable and sensitive materials, interdisciplinary studies are essential to overcome the limitations of SMs and to build promising and reliable sensor devices for their implementation in real-world applications.

Machine learning is considered a favorable tool for developing smart devices with the ability to effectively tackle selectivity and drift problems. Selectivity is a major indicator in defining the sensor performance for both medical and environmental monitoring applications. For example, in the medical field, breath analyzers are used to detect a specific volatile organic compound (VOC) traces among thousands of VOCs in human breath for accurate disease diagnosis. Therefore, it is highly recommended that a breathalyzer should have the ability to detect the traces of specific VOC at certain concentrations with excellent selectivity for proper diagnosis. For environment monitoring, trace-level detection may not be required but excellent selectivity is still a key factor in characterizing the efficiency of a gas sensor.

The past decades have witnessed considerable development in smart gas sensors and electronic noses (e-noses) using machine learning [59-61]. Machine learning mainly contributes to two major factors of a sensing device: drift compensation and selectivity. Over the past few decades, several methods have been investigated to address the drift error through univariate, multivariate, and machine learning [62-65]. Presently, machine learning is being used to tackle both drift compensation and selectivity [66-68]. The machine learning technique involves data processing of sensor output, dimensionality reduction, and then training a system/network for the predictions [66]. Data processing aims to extract robust feature information from the dynamic sensor response, which can represent the unique "fingerprint" patterns for a particular gas to ensure the effectiveness of the subsequent pattern recognition algorithm [66,69]. A number of signal/data processing methods have been reported, such as steady-state (difference, relative difference, fractional difference, normalization, logarithmic difference, etc.), transient (integral and differential), and frequency domain (fast Fourier transform (FFT), continuous/discrete wavelet transform (C/DWT)) models [70]. Among them, the systems/models built on transient and frequency domain data processing/feature extraction methods reveal maximum output accuracy $[69,70]$.

Dimensionality reduction, as the name suggests, is used to reduce the redundancy in high-dimensional preprocessed data. Principal component analysis (PCA), an unsupervised technique, has been extensively studied to improve sensor selectivity (see Appendix A). This is performed by reducing the signal dimension from hundreds of features to only one primary component having the most useful information to generate the unique signatures against the specific gas $[69,71]$. Then, the classifiers/networks are trained and tested on these unique patterns to evaluate the prediction accuracy of unseen preprocessed data. Pattern recognition algorithms are mostly developed using two different approaches: (1) linear classifier using statistical theory and (2) nonlinear classifier using neural network $[59,72]$. Among the commonly used classifiers discussed in the literature [59] are the linear discriminant analysis (LDA), $k$-nearest neighbors (KNN), classification and regression trees (CART), Gaussian naïve Bayes (NB), support vector machines (SVM), random forest (RF), and artificial neural networks (ANN). Recently, Salhi et al. [73] reported a smart early fire detection system using machine learning [73]. They collected 21,146 sample measurements from the sensors under usual and extreme conditions engendering risks and trained their classifiers. Among different supervised machine learning methods, their study estimated the largest accuracy score for CART $(99.93 \%)$ and 
KNN (99.71\%) for the given dataset. They further improved their accuracy rate to $100 \%$ by mean imputation, in which they computed the mean values in the training dataset and replaced them with missing data [73].

This review is divided into two parts: In the first part, we cover the recent developments and limitations of chemresistive gas sensors on the basis of three different kinds of sensing materials, namely, carbon allotropes, 2D transition metal dichalcogenides (TMDCs), and metal oxides (MOx). Different computational and experimental studies are explored to identify the key factors in providing maximum active locations for gas molecule adsorption such as bandgap tuning through different nanostructures, heteroatom doping, nano junction formations, and surface catalytic reaction through hybridization/composition, etc. Afterward, we highlight current limitations in sensor performance such as long-term stability and selectivity. Then, in the second part, machine learning is proposed as a potential approach to efficiently tackle these issues through pattern recognition algorithms. Further, we analyze the significance of different features (static, dynamic, and frequency domain) extracted from response curves of chemiresistive and field-effect transistor (FET) devices for the unique single point signature marker (dimensionality reduction) generation through principal component analysis (PCA) followed by accurate model training. Moreover, the significance of cross-validation techniques for accurate model training is also discussed, specifically manipulation of different k-fold CV methods to enhance the model training, even with the small number of datasets. Finally, the review also discusses recent progress in building highly selective chemiresistive and FET gas sensors and breath analyzers using machine learning.

\section{Chemical Gas Sensors: Achievements and Challenges}

In this section, we focus on the SMs and discuss their structural properties for better sensing. Later, we highlight some computational studies on different kinds of SMs and the critical performance-enhancing factors. Finally, we discuss recent experimental advancements in three different kinds of gas sensors including carbon materials, 2D TMDCs, and MOx.

The development of a chemical gas sensor with fast response/recovery time, maximum sensitivity, minimum aging drift, excellent selectivity, and repeatability are major research concerns and targets. Typically, a sensing material is considered a promising candidate if it possesses a high specific surface area and a highly reactive crystal facet/site for specific gas molecule adsorption with maximum charge transfer $[6,20,25,44]$. It is well documented that bulk metal/MOx materials change their physical and chemical properties entirely when synthesized in micro/nanostructures (NSs). Even by varying the nanostructured morphologies, dramatic change in material properties can be expected. For example, Miller et al. [74] investigated the various defects points on the $\mathrm{SnO}_{2} \mathrm{NPs}$ and NW surfaces using the ultra-high spatial resolution scanning transmission electron microscopy (STEM) combined with cathodoluminescence (CL) system to interpret their role in manipulating the band gap of a nanostructure. They studied four different material samples and found that $\mathrm{SnO}_{2} \mathrm{NW}$ decorated with NPs through sputtering reveals a higher number of defects and thus may enhance the sensor response. However, they proposed that more in-depth investigations under different temperatures and gases (oxidizing/reducing) are required to understand the effect of defects on gas sensing $[45,74]$. Tuning of the surface energy and altering the band gap both can be vital for providing active sites to gas molecules [32,75-78]. Therefore, considerable research works are conducted to develop and explore various micro- (from thick film to highly porous 3D hierarchical structure) and nanostructures (nanoparticles (NPs), nanorods (NRs), nanotube (NTs), nanowires (NWs), and nanocapsules (NCps), etc.) with the aim to build SMs with maximum specific surface area and higher number of active sites. Until now, numerous SMs have been synthesized using physical and chemical routes. Figure 1 displays different types of SMs and their micro/nanostructures with advantages and drawbacks. It also illustrates the significance of hybridization/composition formation to improve the overall performance. In particular, 
it suggests that the decoration of metal/metal oxide NSs on all other types of materials is extremely vital for higher catalytic reaction, depletion region formation ( $\mathrm{p}-\mathrm{n}$ junction and Schottky barriers), to improve response and selectivity. The detailed discussion on different heterostructures ( $\mathrm{p}-\mathrm{n}$ junction, Schottky barriers, and catalyst decoration) and their corresponding performance enhancement mechanisms is not within the scope of this review article. Interested readers are referred to recently published review articles [51,52,79].

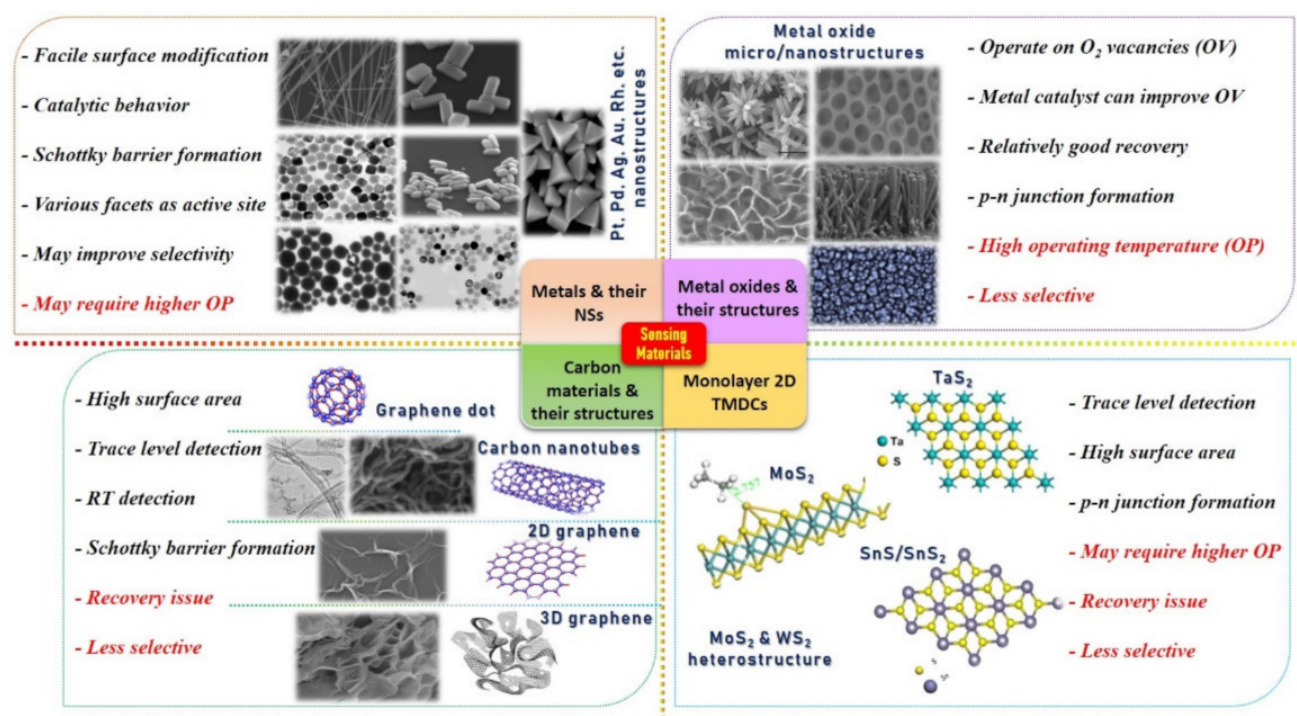

Figure 1. Different kinds of sensing materials with micro/nanostructures, their advantages (in black color), and limitations (in red color), indicating the need of hybridization/composition, doping, and $\mathrm{p}-\mathrm{n}$ junction formation. In particular, the figure suggests that the decoration/doping of metal/hetroatom over the base materials will enhance the catalytic reaction for specific gases and form the charge accumulation depletion region to improve the sensing performances. Reproduced from multiple sources with permission from References [18,41,42,46,80-91]. Copyright 2016 Elsevier [18], copyright 2019 Elsevier [41], copyright 2016 Elsevier [42], copyright 2020 ACS [46], copyright 2020 [80], copyright 2019 Elsevier [92], copyright 2006 ACS [82], copyright 2007 ACS [83], copyright 2013 Elsevier [84], copyright 2014 Elsevier [85], copyright 2016 Elsevier [86], copyright 2018 ACS [87], copyright 2015 Elsevier [88], copyright 2014 Elsevier [89], copyright 2013 Nature [90], and copyright 2012 ACS [91].

Typically, SMs of a resistive-type gas sensor are composed of composite/hybrid materials, categorized as base and catalyst, and each has its role to play for better sensing performances. Base materials are usually responsible for providing a high surface area for gas molecule adsorption and a conductive pathway between two electrodes. This category includes microstructure thick films, thin films, porous films, 3D hierarchical structures, graphene, reduced graphene oxide, 3D graphene, and 2D transition metal dichalcogenides (TMDCs). On the other hand, metal/metal oxide catalysts, synthesized in nanostructures (NSs) and decorated over the base material, are used to enhance the reaction rate on the sensing surface for a specific gas. Therefore, a catalyst is an influential element in improving sensing performances, especially selectivity and response.

\subsection{Computational and Experimental Research}

2.1.1. Identifying Key Performance-Enhancing Factors through Computational Analysis of the Graphene, TMDCs, and Metal Oxides Sensors

Over the decades, great efforts have been devoted to understanding the behavior of the gas molecules on different atomic/molecular sites of the SMs through density function theory (DFT) calculations. It is suggested that adsorption energy, charge transform, and distance between gas molecule and SM surface are highly significant in defining the 
performance ability of a sensor $[25,26,93]$. Gas molecule adsorption site/facet and landing orientation have a great influence on the adsorption energy and maximum charge transfer. High adsorption energy and charge transfer indicate high sensitivity and selectivity towards a specific gas. For instance, Cui et al. [94] studied the layer-dependent sensing performance of phosphorene using computational and experimental investigations. Maximum adsorption energy was noted for $\mathrm{NO}_{2}$ and was confirmed through variations in band structure, signifying higher sensitivity of phosphorene towards $\mathrm{NO}_{2}$ molecules (see Figure 2A). Nevertheless, even for $\mathrm{NO}_{2}$, the charge transfer was not very significant. Therefore, the decoration or doping of metal catalyst over the base SMs is needed to considerably raise the charge transfer and adsorption energy for specific gas [94]. Varghese et al. [95] investigated the gas-sensing properties of boron (B)-, aluminum (Al)-, and gallium (Ga)-doped graphene using DFT calculations. They discovered that B-doped graphene behaved more stable in humidity environment than that of Al- and Ga-doped graphene and displayed adsorption energies of $-0.375 \mathrm{eV}$ and $-1.450 \mathrm{eV}$ for $\mathrm{NO}$ and $\mathrm{NO}_{2}$ molecules, respectively. Furthermore, they calculated maximum adsorption energies for $\mathrm{Al}(-3.474 \mathrm{eV})$ - and $\mathrm{Ga}$ $(-3.050 \mathrm{eV})$-doped graphene towards $\mathrm{NO}_{2}$ molecules in a dry environment [95]. Figure 2B shows a schematic illustration of $\mathrm{NO}_{2}$ molecule adsorption on doped graphene along with its density of state (DOS) for all types of graphene. A clear change in charge distributions at Fermi energy level can be seen for all types of graphene after $\mathrm{NO}_{2}$ molecule adsorption. Likewise, Wang et al. [96] investigated the adsorption of $\mathrm{CO}$ molecules on pure graphene, $\mathrm{N}$-doped graphene, and Al-doped graphene, and their results showed maximum charge transfer of $0.2346 \mathrm{eV}$ between $\mathrm{CO}$ molecule and Al-doped graphene surface [96].

Similarly, iron (Fe)-doped single-layer and bi-layer graphene for $\mathrm{CO}, \mathrm{NO}, \mathrm{SO}_{2}$, and $\mathrm{HCN}$ adsorptions were explored by Tang et al. [97]. Fe-doped bi-layer graphene showed maximum adsorption for NO molecule and weakest for CO. Additionally, the semiconducting and magnetic behavior of SMs after gas molecule adsorption was also studied [97].

Besides graphene, 2D TMDCs and metal oxides have also been investigated using DFT calculations [26,98]. For example, a study by Yue et al. [99] considered the adsorption of different gas molecules including $\mathrm{H}_{2}, \mathrm{O}_{2}, \mathrm{H}_{2} \mathrm{O}, \mathrm{NH}_{3}, \mathrm{NO}, \mathrm{NO}_{2}$, and $\mathrm{CO}$ on pure $\mathrm{MoS}_{2}$ surface. They reported that all the gas molecules were weakly absorbed on $\mathrm{MoS}_{2}$ with less charge transfer, indicating the importance of doping/decoration of metal catalyst (see Figure 3A). They did not further continue their study with doping/decoration of a heteroatom. However, they proposed that the application of a perpendicular electric field promotes gas adsorption on the $\mathrm{MoS}_{2}$ surface [99]. More recently, Qian et al. [80] explored the effect of $\mathrm{Au}$ doping on $2 \mathrm{D} \mathrm{MoS}$ monolayer sheets for $\mathrm{C}_{2} \mathrm{H}_{6}$ and $\mathrm{C}_{2} \mathrm{H}_{4}$ molecule detection. After Au doping, enhancement in adsorption energies and charge transfer between detection molecules and $\mathrm{MoS}_{2}$ monolayer was noted. The maximum adsorption energy and charge transfer were observed for the $\mathrm{C}_{2} \mathrm{H}_{4}$ molecule, finding it to be $-0.952 \mathrm{eV}$ and 0.309 e, respectively. Schematic diagrams are shown in Figure 3B of the Au@MoS surface with adsorbed gas molecules and DOS graphs. These techniques were not only studied in the computational domain but were also extensively investigated and verified through experiments.

Saadi et al. [100] investigated the $\mathrm{NO}_{2}$ sensing mechanism on the $\mathrm{WO}_{3}$ surface, and they observed that the 001 facet of $\mathrm{WO}_{3}$ was more stable and suitable for $\mathrm{NO}_{2}$ sensing. Furthermore, they discussed the significance of oxygen vacancies on metal oxide surfaces and their role in the dissociation of $\mathrm{NO}_{2}$ molecules for the generation of more vacancies, and thus more active sites for the target molecule. DOS results displayed a change in charge distribution at Fermi energy level after $\mathrm{NO}_{2}$ adsorption, indicating the sensitivity of $\mathrm{WO}_{3}(001)$ surface towards $\mathrm{NO}_{2}$ molecules (Figure 2). The schematic illustration and reaction details/equations for $\mathrm{NO}_{2}$ dissociation in $\mathrm{NO}$ and again forming to $\mathrm{NO}_{2}$ are shown in Figure 4A. Bai et al. [101] reported both computational and experimental results of Al-doped $\mathrm{ZnO}$ NSs for $\mathrm{CO}$ sensing. Their experimental and simulated works were well matched and showed enhanced sensing performance towards $\mathrm{CO}$ when ZnO NSs were doped with $\mathrm{Al}$ (Figure 4B). 


\subsubsection{Experimental Progress in Carbon Materials, 2D TMDCs, and MOx-Based} Chemical Sensors

Experimentally, numerous research works have been presented on various materials and methods to improve gas sensing performance. Carbon materials including OD carbon dot, 1D carbon nanotubes (CNTs), 2D graphene, reduced graphene oxide (rGO), and 3D graphene foam/crumpled graphene have been extensively studied for gas sensing due to their high surface area, high electron mobility at room temperature (RT), and high stability and mechanical flexibility [9,17,20,22-24,102]. Pure graphene and rGO have proven to be good candidates for trace level molecule detection at RT but exhibit higher response time with no recovery to baseline $[42,43,102]$. As computational analysis suggests, doping of heteroatom and decoration of metal catalyst dramatically improve the adsorption energy and charge transfer. Therefore, experimentally, those techniques were extensively investigated and proven to be highly promising. For example, sulfur-doped rGO sheets decorated with Ag NPs (10-20 nm) showed an excellent response at RT with full recovery. Fabricated Ag-S-rGO sensor revealed a good response towards $\mathrm{NO}_{2}$ and $\mathrm{NH}_{3}$ with a small response value to some other gasses. Additionally, the sensor displayed $45 \%$ sensitivity towards $50 \mathrm{ppm} \mathrm{NO}_{2}$ concentration with a response/recovery time of $12 \mathrm{~s} / 20 \mathrm{~s}$ [103] (see Figure 5A).

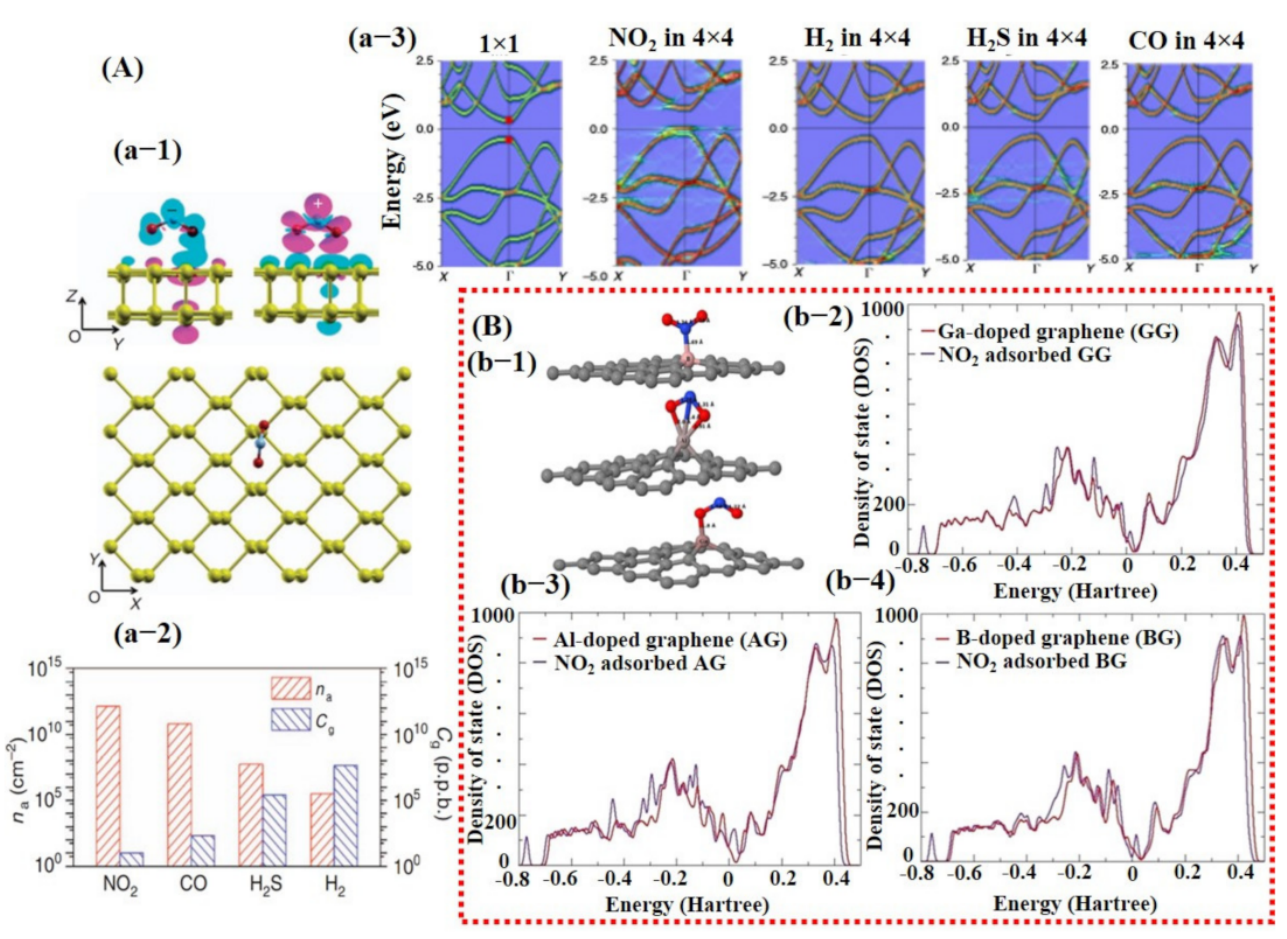

Figure 2. The computational investigation using DFT calculations: (A) DFT calculation for phosphorene: (a-1) shows adsorption of $\mathrm{NO}_{2}$ molecule on phosphorene surface with oxygen atoms pointing downward and the corresponding generated electron and hole clouds; (a-2) left axis indicates the histogram graph (red bars) with adsorption energies of different gases at fixed $20 \mathrm{ppb}$ concentration; (a-3) displays the change in phosphorene band structure after adsorption of different gases including $\mathrm{NO}_{2}, \mathrm{H}_{2}, \mathrm{H}_{2} \mathrm{~S}$, and $\mathrm{CO}$. A clear change on the energy level of conduction band can be observed with the $\mathrm{NO}_{2}$ adsorption on phosphorene surface, reproduced with permission from [94], copyright 2015 Nature. (B) DFT calculations of boron (B)-, aluminum (Al)-, and gallium-(Ga) doped graphene for $\mathrm{NO}_{2}$ detection: (b-1) illustrates a schematic for the $\mathrm{NO}_{2}$ molecule adsorption on all three kinds of graphene surfaces-clear and strong adsorption of $\mathrm{NO}_{2}$ molecule on Al-doped graphene can be seen (middle image); (b-(2-4)) show DOS for all kinds of graphene when exposed to $\mathrm{NO}_{2}-\mathrm{Al}$-doped graphene displayed maximum change at Fermi energy level suggesting its higher sensing ability toward $\mathrm{NO}_{2}$ molecule (b-3). Reproduced with permission from [95], copyright 2016 Elsevier. 
(A)

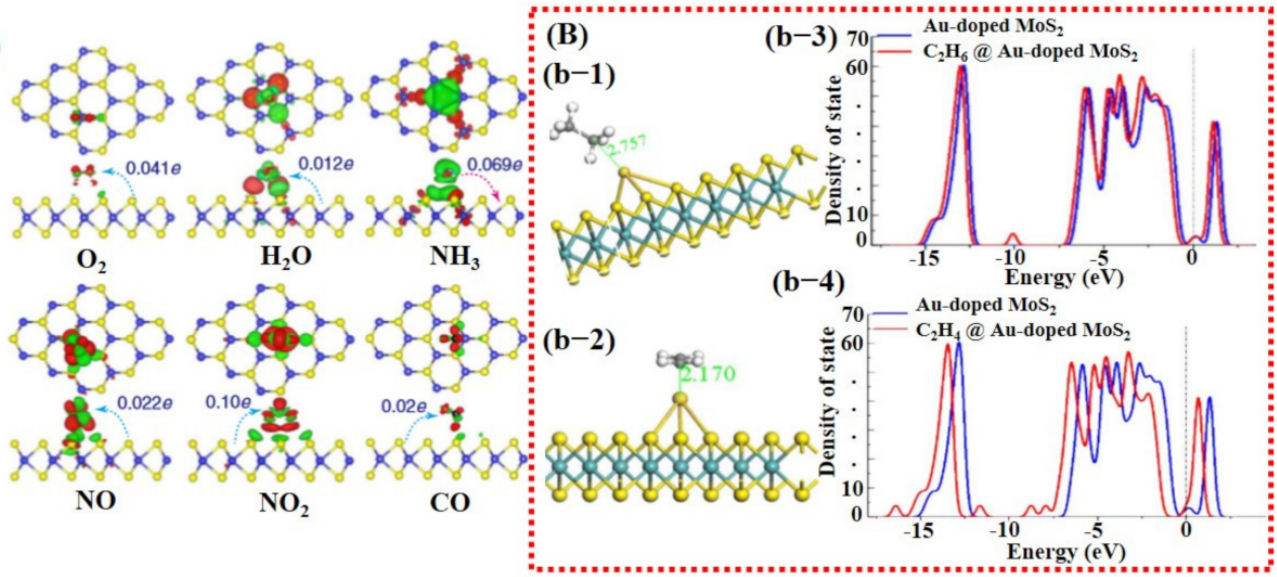

Figure 3. Computational analysis of $\mathrm{MoS}_{2}$ and $\mathrm{Au}$-doped $\mathrm{MoS}_{2}$ for gas sensing using DFT calculations: (A) The charge transfer between pure $\mathrm{MoS}_{2}$ sheet and different target molecules; maximum charge transfer of 0.10 e can be seen for $\mathrm{NO}_{2}$, which is quite low, indicating the significance of heteroatom doping (reproduced with permission from [99], copyright 2013 Springer Nature). (B) The DFT results for Au-doped $\mathrm{MoS}_{2}$ sheet: (b-1) a schematic illustration of $\mathrm{C}_{2} \mathrm{H}_{4}$ and $\mathrm{C}_{2} \mathrm{H}_{6}$ molecule on $\mathrm{Au}$-doped $\mathrm{MoS}_{2}$ with the corresponding bond length distance; (b-3,4) the DOS graphs for both the molecules before and after adsorption. A relatively larger change at Fermi energy level was found for $\mathrm{C}_{2} \mathrm{H}_{4}$, indicating better sensitivity of Au-doped $\mathrm{MoS}_{2}$ towards $\mathrm{C}_{2} \mathrm{H}_{4}$. Reproduced with permission from [80], copyright 2020 Front. Mater.

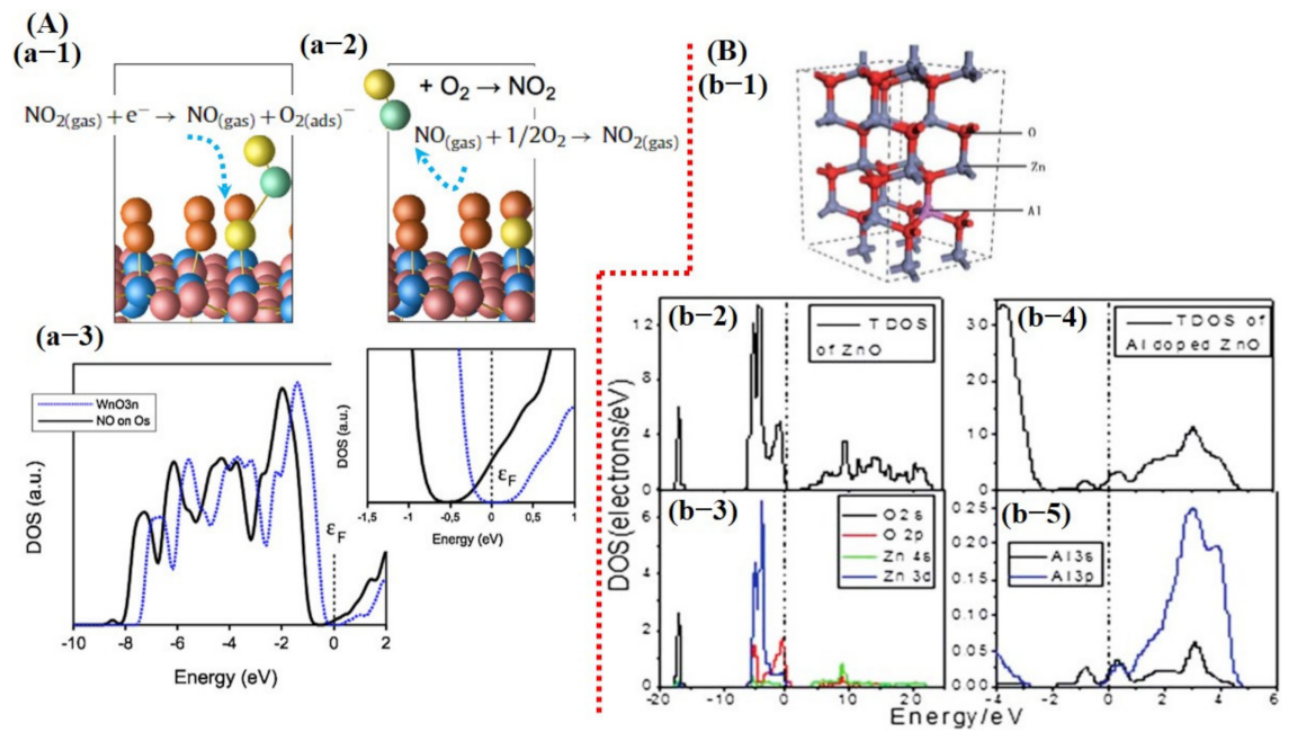

Figure 4. Computational study of metal oxide gas sensors: (A) $\mathrm{NO}_{2}$-sensing mechanism on $\mathrm{WO}_{3}$ surface and DOS graph before and after exposure of $\mathrm{NO}_{2}$ : (a-1) The dissociation of $\mathrm{NO}_{2}$ molecule to $\mathrm{NO}$ when interacting with $\mathrm{WO}_{3}$ surface while leaving behind oxygen adsorbent. (a-2) The formation of $\mathrm{NO}_{2}$ from generated $\mathrm{NO}$ after reacting with one-half oxygen in the environment. This chain cycle significantly improves the oxygen adsorbent population consequently enhances the sensor response. (a-3) The DOS graph. A clear shift can be observed after NO exposure in the magnified image (reproduced with permission from [100], copyright 2014 Elsevier). (B) (b-1) A schematic image of Al-doped $\mathrm{ZnO}$ structure; (b-2) the change in Fermi energy level before and after $\mathrm{Al}$ doping in $\mathrm{ZnO}$ structure (reproduced with permission from [101], copyright 2013 RSC). 

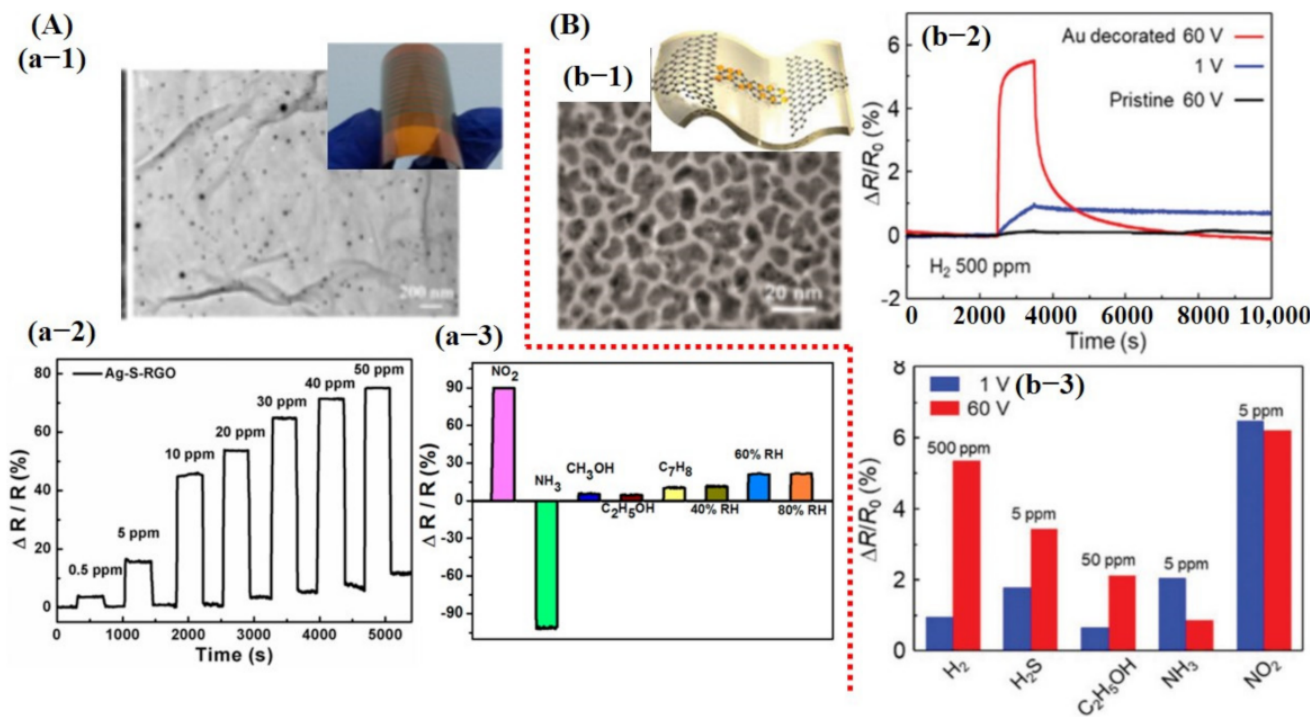

Figure 5. Experimental results of graphene-based gas sensors: (A) Ag NPs decorated on sulfonated reduced graphene oxide (Ag-S-rGO) and their sensing performance for $\mathrm{NO}_{2}$ and $\mathrm{NH}_{3}$ detection: (a-1) SEM image of Ag-S-rGO. Uniformly distributed Ag NPs can be seen on S-rGO surface. (a-2) Sensor response graph towards different concentrations of $\mathrm{NO}_{2}$ gas indicating good sensitivity, fast response/recovery, and linear change in sensor response at different concentrations. (a-3) Response graph to different gases/compounds including $100 \mathrm{ppm} \mathrm{NO}, 1 \mathrm{~mL}$ (13 ppt) of $\mathrm{NH}_{3}, 1 \mathrm{~mL}$ (46 ppt) of methanol, $1 \mathrm{~mL}$ (32 ppt) of ethanol, $1 \mathrm{~mL}$ (18 ppt) of toluene, $40 \% \mathrm{RH}, 60 \% \mathrm{RH}$, and $80 \% \mathrm{RH}$. Reproduced with permission from [103], copyright 2014 ACS. (B) The results for Au @ graphene to detect $\mathrm{H}_{2}$ gas: (b-1) SEM image of Au@graphene; inset shows the schematic image of the device. (b-2) The response of pristine graphene and Au@graphene to 500 ppm $\mathrm{H}_{2}$ gas when $1 \mathrm{~V}$ and $60 \mathrm{~V}$ DC was applied. Sensor shows the maximum response when $60 \mathrm{~V}$ was applied, indicating the importance of biasing voltage. (b-3) A cross-sensitivity graph, suggesting very poor selectivity of the fabricated sensor. Reproduced with permission from [104], copyright 2019 RSC.

Biasing voltage affects the carrier concentration on the sensing surface and it is understood that modulating biasing voltage may play a vital role in better sensing performance. A similar study was carried out by Kim et al. [104]. They developed Au nanocubes (NCs) decorated on a graphene channel for enhanced $\mathrm{H}_{2}$ detection. It was found that after applying $60 \mathrm{~V} \mathrm{DC}$, the deposited Au layer morphology changed to the NCs and enabled better gas sensing at RT. Fabricated sensors displayed noteworthy improvement towards $\mathrm{H}_{2}$ detection after decoration with Au catalyst. However, their sensor did not reveal good selectivity and showed a significant response, even at lower concentrations for other gases [104] (see Figure 5B).

To further improve the selectivity, installation of a separate filter membrane is considered a potential approach for selective detection of a target analyte. In this technique, unwanted gases/chemical mixtures are pre-separated via a filter membrane with a specific pore size to allow the target analyte to pass through and reach the sensor surface. Materials with microporous surfaces such as zeolite, graphene, polymers, and metal-organic frameworks can be widely tuned according to the target analyte size, and are thus considered promising candidates for filter membrane development [105]. Most recently, Jarig et al. chemically synthesized a pore-sized tuned GO filter membrane for selective detection of various VOCs/VSCs. Their fabricated holey GO sheet with various pore sizes displayed superior cross-selectivity to $\mathrm{CH}_{3} \mathrm{COCH}_{3}(0.46 \mathrm{~nm}), \mathrm{C}_{2} \mathrm{H}_{5} \mathrm{OH}(0.45 \mathrm{~nm}), \mathrm{C}_{7} \mathrm{H}_{8}(0.59 \mathrm{~nm})$, and $\mathrm{H}_{2} \mathrm{~S}(0.36 \mathrm{~nm})$. The synthesized holey $\mathrm{GO}$ filter membrane was placed over sensing material ( $\mathrm{PdO}-\mathrm{WO}_{3}$ nanosheets) that act as a molecular sieving layer to selectivity pass target analyte according to the pore size distribution on its surface. They claimed that a pore size-tuned GO porous layer is promising for designing low-cost and highly efficient 
gas sensors with outstanding selectivity [105]. However, these filter membranes might be restricted to very small size analyte detection as the bigger pores may allow for diffusion of several other gases/chemicals with similar diameters, resulting in poor selectivity.

$\mathrm{Wu}$ et al. [39] reported a high-performance $\mathrm{NO}_{2}$ sensor at $\mathrm{RT}$ using boron (B)- and nitrogen $(\mathrm{N})$-doped 3D reduced graphene oxide hydrogel $(\mathrm{RGOH})$. Their fabricated sensor displayed significant improvements. The sensing performance details are shown in Figure $6 \mathrm{~A}$ and are discussed in the introduction section of this article.

Likewise, Phan et al. [81] reported a fast response and highly sensitive $\mathrm{H}_{2}$ sensor using $\mathrm{Pt}$ NPs decorated on 3D graphene. Their sensor showed a response value of $16 \%$ with response/recovery times of $9 / 10$ s to $1 \% \mathrm{H}_{2}$ concentration at an OT of $200{ }^{\circ} \mathrm{C}$. Moreover, they claimed good stability and linearity towards different gas concentrations. However, they did not report the selectivity of their fabricated sensor (see Figure 6B).
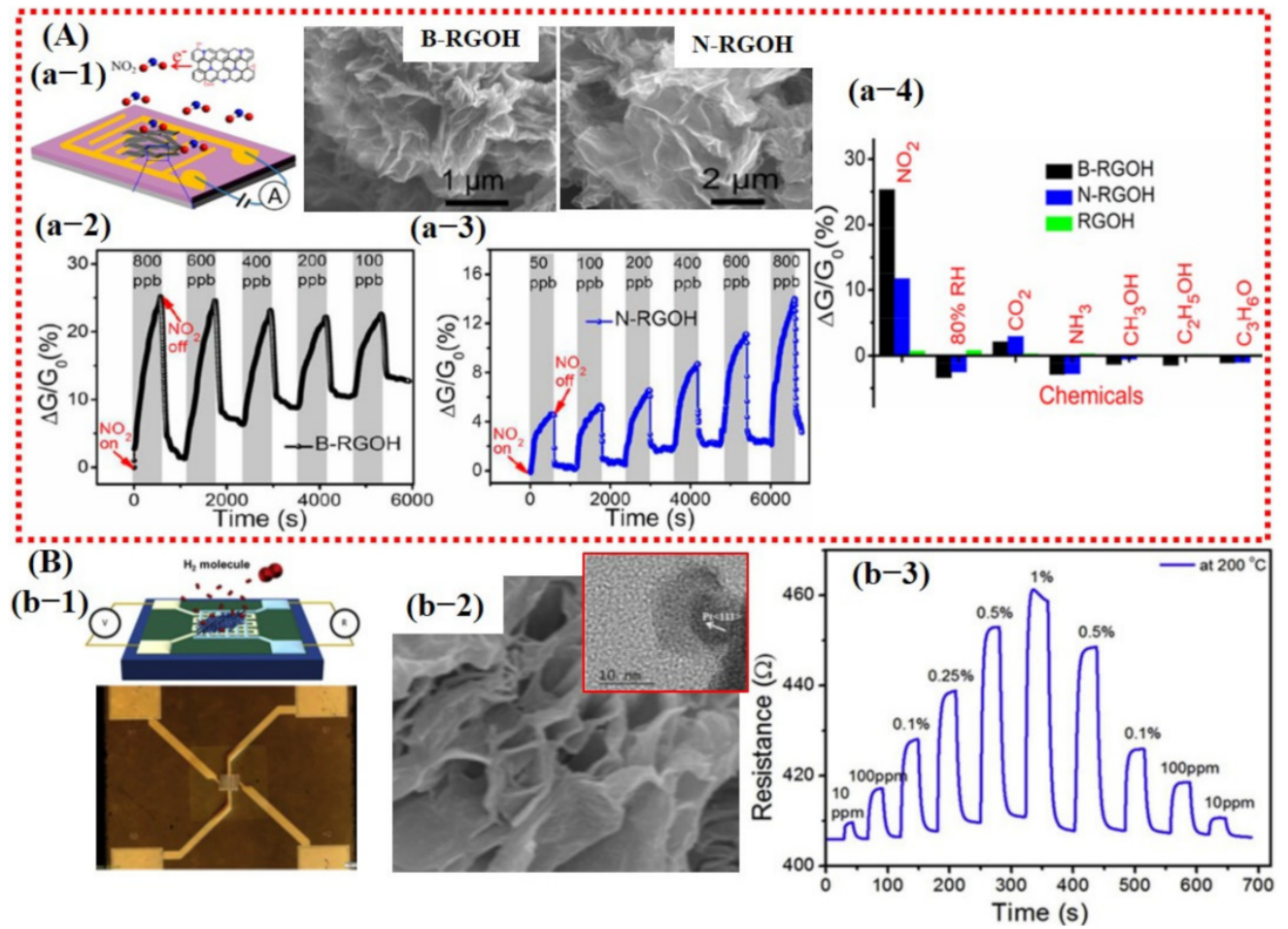

Figure 6. Representation of the sensing performances of 3D graphene: (A) Sensing results of Band N-doped RGOH: (a-1) Schematic diagram of the fabricated sensor device with SEM images of highly porous B- and N-doped graphene. (a-2,3) The response of B-RGOH and N-RGOH at different concentration levels of $\mathrm{NO}_{2}$ gas. Results show that $\mathrm{N}-\mathrm{RGOH}$ revealed a good response at lower $\mathrm{NO}_{2}$ concentration. (a-4) Responses of the B- and $\mathrm{N}-\mathrm{RGOH}$ to $800 \mathrm{ppb} \mathrm{NO}, 80 \% \mathrm{RH}, 1000 \mathrm{ppm}$ $\mathrm{CO}_{2}, 100$ ppm $\mathrm{NH}_{3}$, saturated methanol, ethanol, and acetone vapors. Reproduced with permission from [39], copyright 2019 ACS. (B) The sensing performances of Pt decorated over highly porous 3D graphene: (b-1) An optical and schematic image of fabricated sensor device. (b-2) SEM image of highly porous 3D graphene; inset shows the TEM image of Pt NP. (b-3) The symmetric response of Pt-3D graphene to various $\mathrm{H}_{2}$ concentration levels at $200{ }^{\circ} \mathrm{C}$. Reproduced with permission from [81], copyright 2019 Elsevier.

Due to the exceptional performances of 2D graphene/rGO-based sensors, efforts were then made to work on other 2D materials with some band gap, including $\mathrm{MoS}_{2}$, $\mathrm{MoSe}_{2}, \mathrm{SnS}_{2}, \mathrm{VS}_{2}, \mathrm{TaS}_{2}$, and $\mathrm{WS}_{2}$ for the fabrication of transistor-based devices $[6,25,26]$. Among them, $\mathrm{MoS}_{2}$ was widely studied to explore the sensing performances of TMDCs. At first, transistor devices were made using pure $\mathrm{MoS}_{2}$ monolayer and then later on for further enhancement in sensing performance hybridization of the $\mathrm{MoS}_{2}$, with metal/metal oxide catalyst being investigated. For instance, the sensors made of pure $\mathrm{MoS}_{2}$ showed 
little response towards different gases but were not able to display full recovery and good selectivity [106]. Park et al. [107] reported enhanced $\mathrm{NH}_{3}$ and $\mathrm{H}_{2} \mathrm{~S}$ detection using $\mathrm{Pt}$ NPs decorated on $\mathrm{MoS}_{2}$-synthesized via vapor deposition technique. Their sensor revealed relatively better performance than that of pure $\mathrm{MoS}_{2}$ sensors but still did not achieve full recovery to the baseline (see Figure 7A). Moreover, they did not report crosssensitivity with other gases. $\mathrm{MoS}_{2}$ synthesized via physical route did not reveal good performance [106,107]. Therefore, chemically synthesized $\mathrm{MoS}_{2}$ sheets were investigated by Burman et al. [92]. They reported Pt NPs decorated on chemically synthesized $\mathrm{MoS}_{2}$ flakes for humidity sensing. Fabricated sensor loaded with $25 \%$ Pt NPs showed a maximum response. The sensors demonstrated excellent response towards different percentages of humidity with full recovery to baseline, but they too did not report selectivity of their sensors. To check the long-term stability, they tested the sensor after 1.5 months, and a clear degradation in response was observed indicating the highly unstable nature of the synthesized material [92]. The sensing results for the fabricated sensor are summarized in Figure 7B.

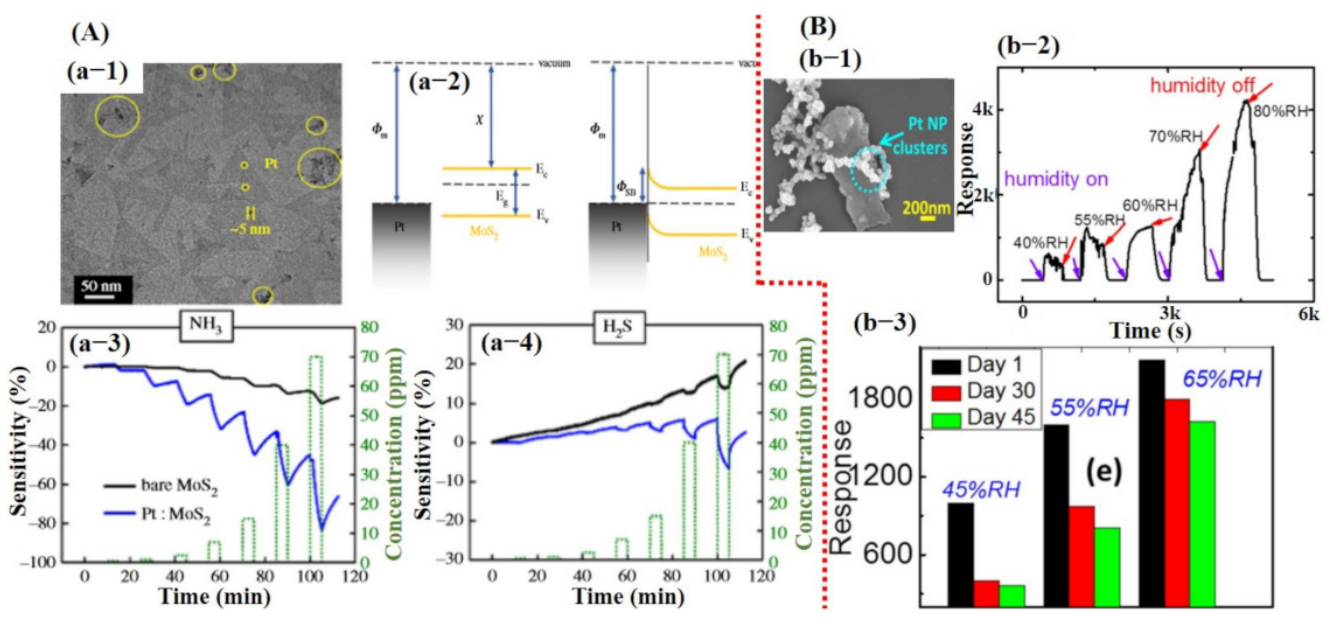

Figure 7. Demonstration of the sensing results for physically and chemically synthesized $\mathrm{MoS}_{2}$ sheets: (A) Sensing properties of Pt NPs decorated on physically grown $\mathrm{MoS}_{2}$ for $\mathrm{NH}_{3}$ and $\mathrm{H}_{2} \mathrm{~S}$ detection: (a-1) SEM image of monolayer $\mathrm{MoS}_{2}$ decorated with Pt NPs. (a-2) The formation of Schottky barrier between $\mathrm{Pt}-\mathrm{MoS}_{2}$ and barrier height. (a-3,4) Sensor response towards the $\mathrm{NH}_{3}$ and $\mathrm{H}_{2} \mathrm{~S}$. Reproduced with permission from [107], copyright 2018 Royal society. (B) The sensing performance of Pt NPs decorated on the chemically synthesized $\mathrm{MoS}_{2}$ sheets towards humidity: (b-1) SEM image of Pt NPs decorated on $\mathrm{MoS}_{2}$. The inset reveals a higher magnified image. (b-2) Sensor response towards different levels of relative humidity. The sensor showed a good response with full recovery, revealing that a chemically synthesized $\mathrm{MoS}_{2}$ is a promising candidate. (b-3) Stability test was checked after 1.5 months, and degradation in sensor response was observed. Reproduced with permission from [92], copyright 2018 IOP.

Besides carbon materials and TMDCs, metal oxide was also broadly investigated through various techniques, such as hybridization, $\mathrm{p}-\mathrm{n}$ junction formation, and core-shell structures $[27,28,37,50,108]$. As discussed in the computational section, oxygen vacancies are vital for metal oxide-based sensors. The type and number of oxygen vacancies depend upon the OT $\left(\mathrm{O}_{2}{ }^{-}, \mathrm{O}^{-}\right.$, and $\left.\mathrm{O}^{2-}\right)$. Usually, sensors perform well at high temperatures due to the higher number of oxygen vacancies and higher reaction rate between the target molecule and sensing material surface [45]. Likewise, for carbon and TMDCs sensors, the decoration of metal catalysts was needed to further improve the sensor performance together with selectivity.

Kolmakov et al. [109] fabricated sensors using Pd catalyst decorated on a single $\mathrm{SnO}_{2}$ NW. They indicated enhanced sensing response to oxygen and $\mathrm{H}_{2}$ gases. To understand the catalytic effect of Pd, they observed I $\mathrm{DS}$ current value during Pd deposition. At the start, 
a reduction in $\mathrm{I}_{\mathrm{DS}}$ value was noted, which indicated that the Pd NPs on the NW surface created Schottky barrier-type junctions resulting in the formation of electron depletion regions within the NW. However, at certain Pd deposition times, a dramatic increase in conductance was observed, representing percolation among the NPs, eventually leading to shorting of the $\mathrm{SnO}_{2} \mathrm{NW}$. They explained that the formation of depletion zones through the Schottky barrier plays a vital role to enhance the sensor performance by increasing the population of oxygen vacancies in that particular region [109] (see Figure 8A). Therefore, considerable research is being devoted to attaining the depletion zone through the Schottky barrier, $\mathrm{p}-\mathrm{n}$ junction formation, layer by layer, and core-shell NSs $[34,48,50,52,58,109,110]$.

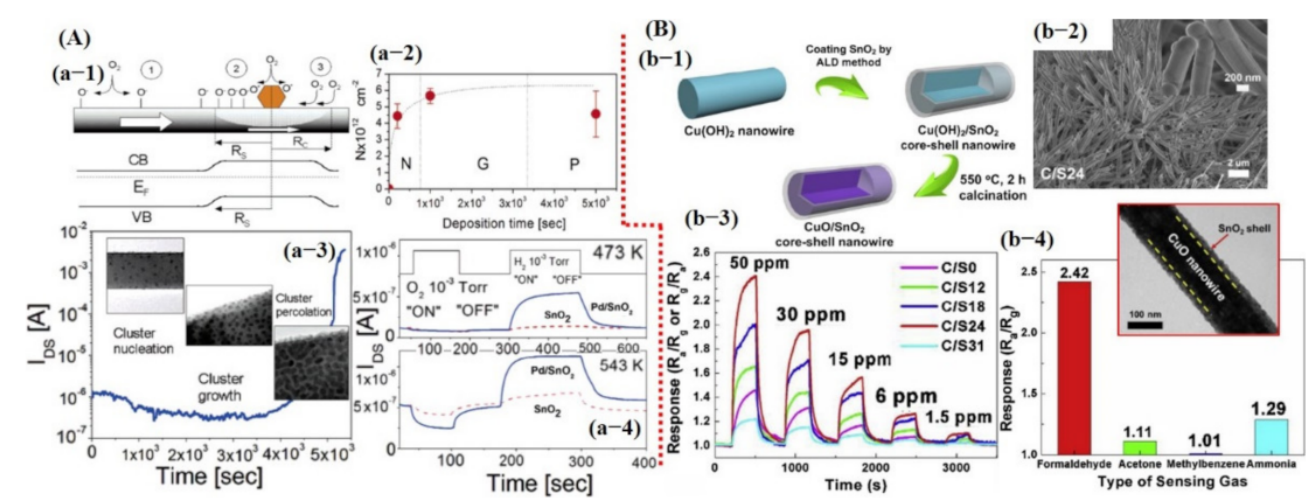

Figure 8. Metal oxide-based gas sensors: (A) Pd NPs decorated on the single $\mathrm{SnO}_{2} \mathrm{NW}$ for $\mathrm{O}_{2}$ and $\mathrm{H}_{2}$ detection: (a-1) The formation of the depletion region on the nanowire (NW) surface with the Pt NP deposition. As shown in Figure 8 (a-1), the depletion region facilitated in increasing the oxygen adsorbent on the sensing material and consequently improved the sensor performance. (a-2) The Pt NP deposition rate. (b-3) IDS was measured during Au deposition. The onset of the large current increased beyond $\approx 4 \times 10^{3} \mathrm{~s}$ can be attributed to the percolated pathways generated through excessive Au particles. (a-4) The sensing performances of pure $\mathrm{SnO}_{2}$ and $\mathrm{Pd}-\mathrm{SnO}_{2} \mathrm{NW}$ to $\mathrm{O}_{2}$ and $\mathrm{H}_{2}$. Reproduced with permission from [109], copyright 2005 ACS. (B) Sensing performance of ${\mathrm{CuO} @ \mathrm{SnO}_{2}}_{2}$ core-shell structure for formaldehyde detection. (b-1) Schematic diagram of the fabrication process. (b-2) SEM image of core-shell NW structure. (b-3) Sensor response with different shell thickness; $\mathrm{SnO}_{2}$ with $24 \mathrm{~nm}$ thick shell revealed maximum response. (b-4) Selectivity graph. The inset revealed the TEM image of the core-shell NW structure. Reproduced with permission from [111], copyright 2019 Elsevier.

Recently, Zhu et al. [111] fabricated hetero-structured $\mathrm{p}-\mathrm{CuO} / \mathrm{n}-\mathrm{SnO}_{2}$ core-shell NWs by combining a solution and atomic layer deposition process for enhanced sensitive and selective formaldehyde detection. They indicated that $\mathrm{SnO}_{2}$ shell thickness is crucial; $24 \mathrm{~nm}$ thick $\mathrm{SnO}_{2}$ shell showed a high sensitivity of 2.42, a threefold higher response than that of pristine $\mathrm{CuO}$ NW towards $50 \mathrm{ppm}$ formaldehyde $(\mathrm{HCHO})$ at $250{ }^{\circ} \mathrm{C}$. The enhanced gas sensing performance could be attributed to the formation of $\mathrm{p}-\mathrm{n}$ heterojunction through specific band alignment and the heterojunction-depletion model. The cross-sensitivity was also checked with three other gases/compounds; $\mathrm{NH}_{3}$ showed a comparatively higher response of 1.29 than that of acetone and methylbenzene [111] (see Figure 8B).

Similarly, $\mathrm{Li}$ et al. [57] reported a $\mathrm{Pd}-\mathrm{Au}$ nanoparticle (NPs) decorated on $\mathrm{SnO}_{2}$ nanosheets (NShs) for temperature-dependent acetone and formaldehyde detection. $\mathrm{Xu}$ et al. [58] also reported Ag@Pt core-shell nanostructures (NSs) decorated on $\mathrm{ZnO}$ nanowires (NWs) for formaldehyde detection. Their results are shown in Figure 9A,B and discussed in the introduction section of this article. 


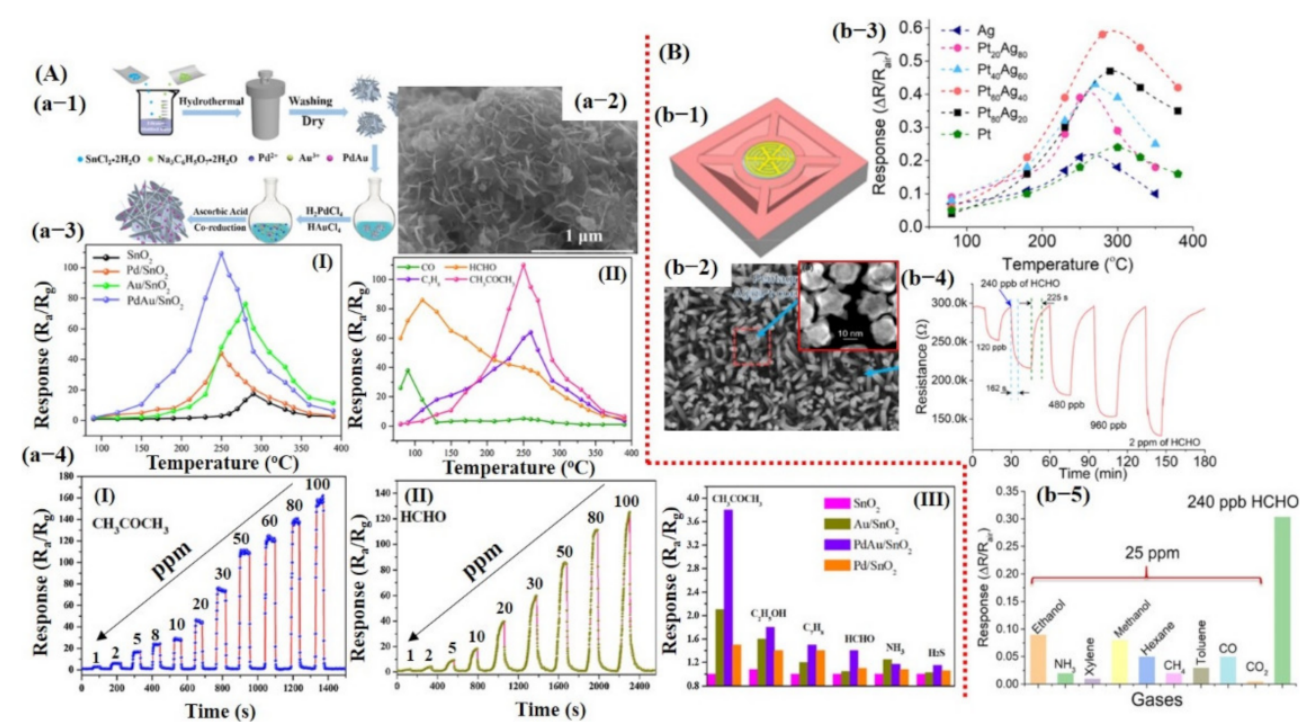

Figure 9. Metal oxide-based gas sensors: (A) Pd/Au NPs decorated on the $\mathrm{SnO}_{2}$ nanosheets for temperature-dependent acetone and HCHO detection: (a-1) Synthesis process of Pd/Au NPs decorated on the $\mathrm{SnO}_{2}$ nanosheets. (a-2) The SEM image for the $\mathrm{Pd} / \mathrm{Au}$-decorated $\mathrm{SnO}_{2}$ nanosheets. (a-3) The temperature-dependent response of the as-fabricated sensors: (I) sensing performance of $\mathrm{SnO}_{2}$, $\mathrm{Pd} @ \mathrm{SnO}_{2}, \mathrm{Au} @ \mathrm{SnO}_{2}$, and Pd/Au@SnO 2 to 50 ppm acetone, with Pd/Au@SnO 2 demonstrating maximum response at $250{ }^{\circ} \mathrm{C}$; (II) $\mathrm{Pd} / \mathrm{Au} @ \mathrm{SnO}_{2}$ sensor response towards various compounds (50 ppm) at different OT, showing maximum response for $\mathrm{HCHO} @ 110^{\circ} \mathrm{C}$ and acetone @ $250{ }^{\circ} \mathrm{C}$. (a-4 (I, II)) The sensor response @ $250^{\circ} \mathrm{C}$ and $110^{\circ} \mathrm{C}$ for a wide range of acetone and $\mathrm{HCHO}$ concentration levels, respectively. (a-4 (III)) The responses toward 1 ppm acetone compared to 1 ppm of the other common interfered biomarker gases. Reproduced with permission from [57], copyright 2019 Elsevier. (B) Ag@Pt core-shell NSs on the ZnO NWs for HCHO detection: (b-1) Fabricated chemiresistive sensor device. (b-2) SEM image of Ag/Pt core-shell on ZnO NWs. The inset reveals the magnified image of Ag@Pt core-shell NSs. (b-3) Sensor response by varying the Pt and Ag content ratio. The Pt60 and Ag40 showed a maximum response at $280{ }^{\circ} \mathrm{C}$ and were selected for further measurements. (b-4) Optimized sensor response toward different concentration levels of HCHO. (b-5) Sensor response to other compounds. Reproduced with permission from [58], copyright 2020 ACS.

\subsection{Current Challenges in Chemical Gas Sensors}

From the aforementioned state of art survey, one can see that the explored materials and techniques can certainly assist in achieving the milestone for maturation of the sensor industry. These techniques significantly enhance the sensing properties in terms of sensitivity, response/recovery time, repeatability, the limit of detection (LOD), and OT. However, excellent selectivity with long-term stability is still a great challenge. Materials tend to lose their properties with the environmental effects and aging, thereby causing degradation in the sensor response, which is known as the drift error. Most of the studies did not consider the aging effect (long-term stability) which is essential for sensor implementation in a real-world application. On the contrary, great efforts have been devoted to developing a sensing material for highly selective detection of a particular gas/compound. The reported sensors demonstrated higher sensitivity towards a specific gas/compound; however, were not able to completely neglect the other gases and showed cross-sensitivity with small response values.

Additionally, the metal NSs, which are considered and reported as good catalysts for a particular gas, have been used for the selective detection of various other gases, showing significant improvement in selectivity. For instance, $\mathrm{Pt}$ is considered the best catalyst for $\mathrm{H}_{2}$ sensing but it is also used for other gases/compounds and has shown competitive results. Pt NPs decorated on physically grown $\mathrm{WO}_{3}$ NRs showed high sensitivity towards $\mathrm{H}_{2}$ gas $[112,113]$. Pt NPs of a similar material decorated on physically synthesized $\mathrm{WO}_{3}$ 
hemitube [114] demonstrated a good response to $\mathrm{H}_{2} \mathrm{~S}$ and acetone, suggesting in-depth investigation for proper understanding of NSs effect on sensing even with same material synthesized in similar manners. Pt decorated on $\mathrm{WO}_{3}$ synthesized via the same physical route showed promising sensing performance for $\mathrm{NO}$ [115] and $\mathrm{SO}_{2}$ sensing [116]. Similarly, $\mathrm{Au}$-doped $\mathrm{ZnO}$ gas sensors showed a good response to a number of gases including $\mathrm{NH}_{3}$ [117], $\mathrm{CO}$ [118], and HCHO [110]. Recently, nanosized Au loaded on ZnO NPs was developed for toluene detection, achieving ultrasensitive output performances [48]. Furthermore, Ni-doped $\mathrm{ZnO}$ demonstrated a good sensing response towards $\mathrm{NH}_{3}$ [119] and $\mathrm{HCHO}$ [120], leaving a big question on the selectivity. Therefore, in-depth analysis and investigation are needed to apprehend the surface charge distribution on various facets of sensing material nanostructures (nanoparticles, nanopyramid, nanoporous, nanocubes, nanowires, nanorods, nanotubes, etc.) through computational (to find a site/facet that allows maximum adsorption and charge transfer between target molecules and surface) and experimental studies.

In parallel with the development of novel material, it is equally indispensable to find facile, efficient, and smart alternatives to improve the selectivity and to compensate for the drift error without compromising other sensor performances

\section{Machine Learning-Based Smart Gas Sensors}

Machine learning has emerged as a potential data-processing approach to improve selectivity and to compensate for drift error. However, it requires a large amount of labeled data under different circumstances for accurate training of classifier models [59,66,121]. A large dataset (with hundreds/thousands of examples) of a sensor response to a particular analyte will probably result in high redundancies due to the co-existence of data points. Therefore, a careful selection and gradation of important features are highly recommended using variance analysis test (ANOVA F-test) and heat maps in order to avoid the data overlapping. The obtained important feature vectors are used as an input to the PCA for the generation of a unique signature marker for a specific analyte. Although PCA further reduces the dimensionally, all the information from the input features are efficiently retained by projecting the data in reference space with best fit linear slop. The feature variance across the linear fit curve is the obtained first principal component of the input data. Figure 10 illustrates the overall machine learning process including feature extractions through various data-processing techniques and different linear/nonlinear models and their training methods for accurate prediction. As indicated, initially it is essential to identify the useful features from the dynamic response curves (steady-state, transient, and frequency domain), process them using an unsupervised PCA algorithm for dimensionality reduction, and visualize the unique signature pattern against each class. Afterward, the models are trained on the obtained signature patterns through appropriate cross-validation (CV) approaches for the realization of the best fit model with maximum accuracy. The final step is the accuracy testing of the trained models on the unknown dataset [122-126]. Various kinds of features and their extraction techniques for chemiresistive and FET sensor devices are discussed in the following sections. Principal component analysis (PCA) is one of the most widely used unsupervised techniques in the literature. The main contribution of this technique is to generate unique fingerprints and reduce the computational power during model training by eliminating the redundancy of a big dataset through the diagonalization of the correlation matrix. Appendix A presents basic information on PCA calculations. During model training, the optimization of the model algorithms through different $\mathrm{CV}$ methods is essential to avoid overfitting and to acquire maximum and stable prediction accuracy rates. The CV is a statistical method used to approximate the accuracy of a machine learning model. The hold-out and $k$-fold (stratified $k$-fold $\mathrm{CV}$, leave-one-out (LOOCV), leave p-out (LPOCV), etc.) are the commonly reported CV techniques [122, $125,126]$. The selection of an appropriate CV technique for a particular dataset is the key to develop a highly efficient machine learning model. The hold-out is one of the most basic and simple approaches in which the entire dataset is first randomly shuffled and 
then divided into two datasets: training and testing (for training and validation of model accuracy, respectively), as shown in Figure 11. Mostly, the training dataset (60-80\%) ratio is set higher than the testing dataset (40-20\%) in order to increase the model training probability rate on all available examples in the dataset. However, random shuffling of the dataset may alter the whole training process and thus reveal unstable prediction accuracy. Additionally, it is not suitable for a small amount of datasets since it randomly shuffles the entirety of the data into two subsets (training and testing), and it is highly likely that the model may not be trained on all existing examples in the dataset. As a result, it may lose accuracy. The hold-out approach is considered more suitable for a large dataset in which the possibility of model training on all examples will be much higher with relatively lower computational power requirement during model training. On the contrary, the $k$-fold CV is considered a promising approach for comparatively smaller datasets and to address the hold-out limitations. In this method, the entire dataset is split into $k$ subsets, in which every data point will take part in training and testing of the model, as shown in Figure 11. To achieve the training and testing at each data subset, the machine learning algorithm runs for $k$ iterations (Figure 11). The accuracy of the model is calculated at each iteration and then averaged after the $k$ th iteration to attain the final accuracy rate of the model. Since this $\mathrm{CV}$ method trains and tests the model at each data point, the probability rate for training on each example will be much higher, even with small datasets. The selection of $k$ value is highly sensitive to the nature of the input dataset for accurate training of a model. The implementation, advantages, and accuracy of these techniques for various chemiresistive and FET sensors/e-nose systems are covered in the next section.

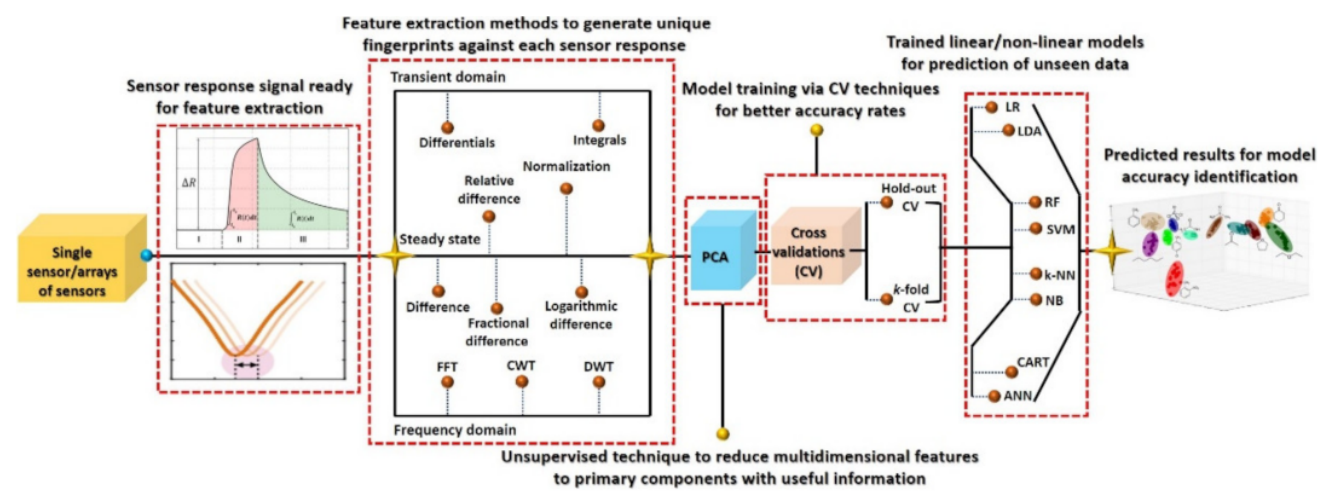

Figure 10. Overall machine learning process from sensors response, data processing, and model training to prediction accuracy.

In the past few decades, numerous studies have been conducted on electronic nose (e-nose) systems, which consist of an array of different gas sensors that interact with a broad range of chemicals of varying strengths, feature extraction, and pattern recognition algorithms that process and extract useful information to generate unique fingerprints [70,127,128]. Recently, Salhi et al. [73] developed a whole hardware setup (from sensing to training and testing of different classifiers) for early gas leakage detection in smart homes (see Figure 12). They divided the setup into three logical layers: node layer, gateway layer, and application layer. The node layer mission is to collect and process the data, then transmit it through a low-power wireless network (LPWN) such as ZigBee, Z-Wave, and Bluetooth devices to the gateway layer. The gateway layer collects the data from the node layer and can be accessed remotely by the end-user as a control and monitoring system. Data are then examined by the application layer. This layer serves as the interface between machine to machine (M2M) home network and M2M devices. The main purpose of this layer is to evaluate and correlate data received from the gateway layer to detect anomalous patterns; for instance, predicting gas leakage and fire incidences in a smart home environment [73]. Their e-nose consists of seven commercial sensors, including temperature, humidity, $\mathrm{LPG}, \mathrm{CO}, \mathrm{CO}_{2}$, smoke, and flame. Data were collected 
under usual and extreme conditions during 1.5 days, and 21,146 measurement samples were obtained. Each sample counts seven values, i.e., one value for each sensor. Every sample is collected periodically every $5 \mathrm{~s}$. In the study, six classifiers were trained using 10-fold cross-validation (CV) with the existing dataset (training $80 \%$ and testing $20 \%$ ): logistic regression (LR), linear discriminant analysis (LDA), $k$-nearest neighbors (KNN), classification, regression trees (CART), Gaussian naïve Bayes (NB), and support vector machines (SVM). Their results revealed the largest estimated score using CART (99.93\%) and with KNN (99.71\%) accuracy for the given dataset. They further improved the accuracy by computing the mean values in the training dataset and replacing them with missing data [73]. Though they were able to develop very selective smart sensors with almost $100 \%$ accuracy, their setup was very large, relatively costly, and consumed more power [73]. The overall setup collected data, and the result is shown in Figure 12.

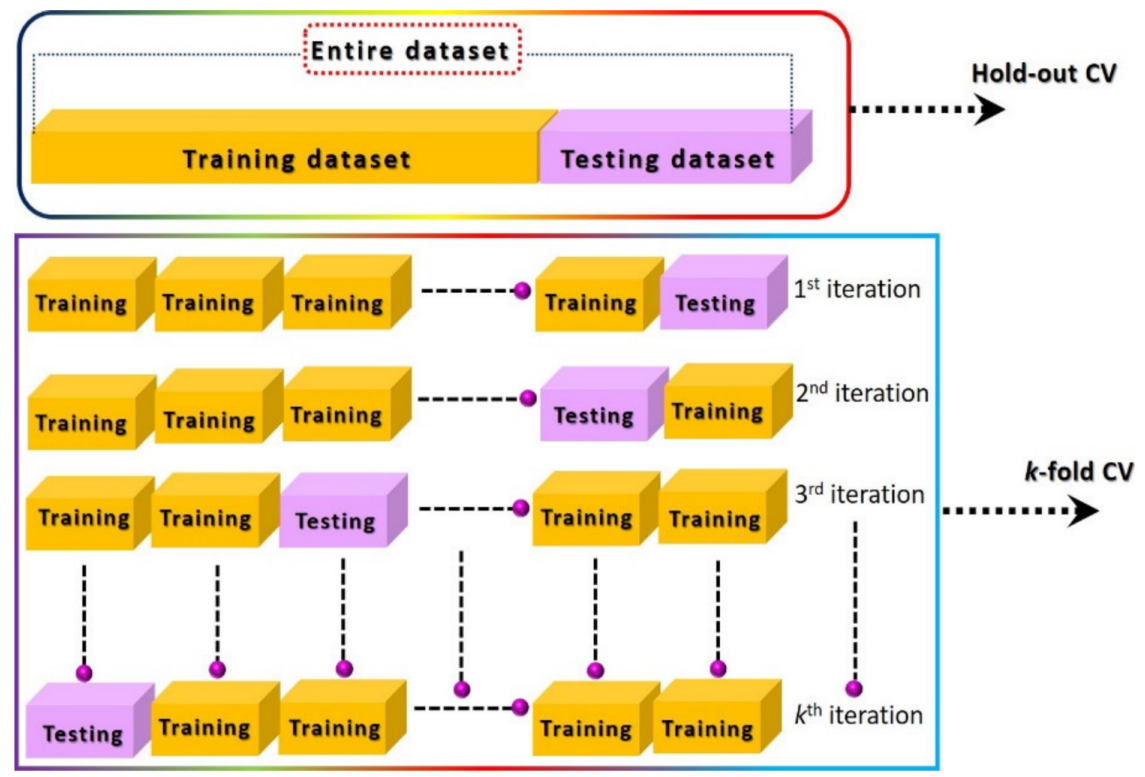

Figure 11. Illustration of hold-out and $k$-fold cross-validation techniques.
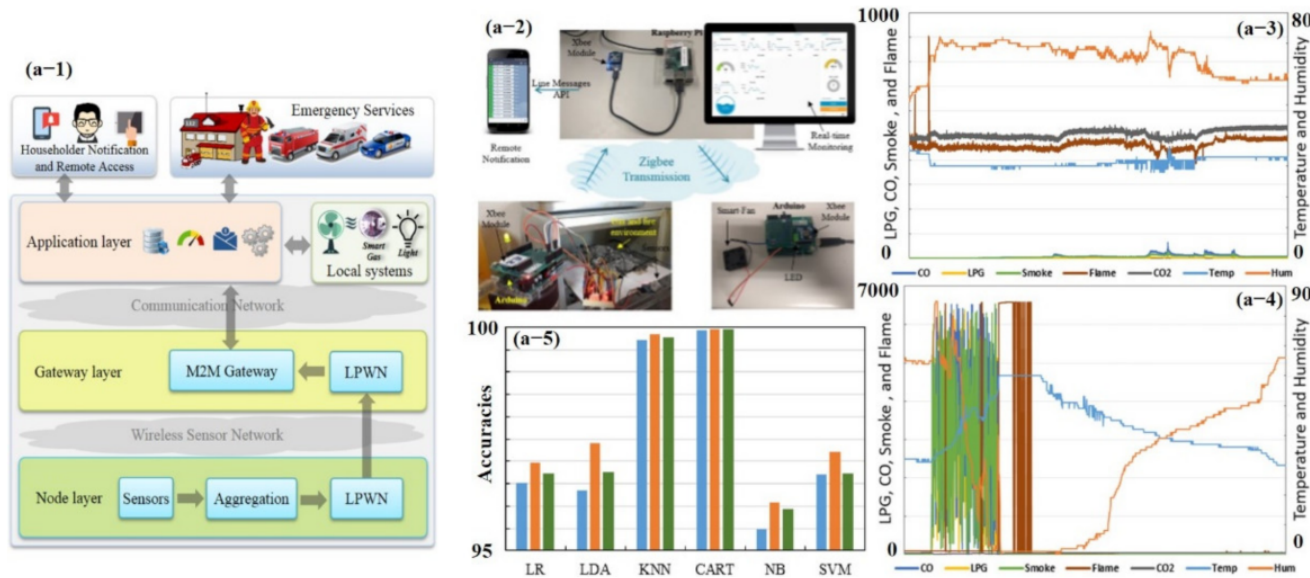

Figure 12. Early fire detection in smart homes using machine learning: (a-1) overall system overview, (a-2) whole hardware and experimental setup, (a-3) sensor measurements taken under usual conditions, (a-4) sensor measurements taken under extreme conditions, and (a-5) prediction accuracy histogram before and after replacing the missing values with the mean values computed from the training data. Reproduced with permission from [73], copyright 2020 IEEE. 
To reduce the power consumption and to develop an economical setup, researchers fabricated and reported single-sensors based on selective material synthesized through earlier mentioned techniques [124,129]. These sensors generate distinct dynamic responses against different gases, and hence one sensor can be used for the identification of various gases. The details are discussed in the coming sections.

\subsection{Chemiresistive Type Smart Gas Sensors Using Machine Learning}

Enormous efforts have been made to improve the sensing ability of chemiresistivetype sensors using various machine learning techniques. A typical resistive type gas sensor shows a change in resistance upon exposure of gas molecules. An increase/decrease in resistance depends on the nature of the sensing material and target molecule [42,43]. An output dynamic response signal with appropriate labeling of baseline, response time, and recovery time is shown in Figure 13Aa-1. Performance enhancement of a smart sensor mainly depends on three factors: (1) use of appropriate sensing materials, (2) useful feature extraction and data processing techniques, and (3) efficient training of single model/multiple models. It has been reported that model/classifier accuracy can be significantly improved using cascading of multiple classifiers. For example, Guney et al. [122] reported classification of n-butanol concentrations using k-NN and SVM. The decision tree structure was used to extract the features, and then K-NN and SVM classifiers were trained on these features using the leave one out (LOOCV) technique. The LOOCV method is a type of $\mathrm{k}$-fold $\mathrm{CV}$ where $\mathrm{k}=\mathrm{N}$ and $\mathrm{N}$ is the total number of the data points. This method certainly helps to improve the accuracy rate but it is recommended for small datasets as the number of iterations is equal to the number of data points. Thus, for bigger datasets, it will require higher computational power and time to train a model. The K-NN (93\%) and SVM (96\%) classifiers with decision tree models showed great improvement in accuracy compared with singular SVM (86\%) and K-NN (87\%) classifiers [122].

For one to obtain a PCA graph with great discriminating ability among different classes, a careful selection and gradation of important features is highly recommended using a variance analysis test (ANOVA F-test) and heat maps in order to avoid the data overlapping. For example, Faleh et al. [131] studied the recognition of ozone $\left(\mathrm{O}_{3}\right)$ using an array of four $\mathrm{WO}_{3}$ sensors and PCA calculations. They reported that the static parameter $\mathrm{R}_{\text {gas }} / \mathrm{R}_{\text {air }}$ is not sufficient to distinguish among various concentrations of the target gas. Therefore, for better discrimination among various concentrations, they used the area under the response time curve from the dynamic (transient) response. It was concluded that using the response time parameter, the class separation among different concentrations was much better than the resistance ratio [131]. Later, Nallon et al. [123] used unmodified graphene as a single sensor for discrimination among different chemicals/compounds. Their fabricated sensor was successfully able to differentiate among 11 different compounds without any considerable overlapping. Excellent PCA results can be attributed to the features selected with the most useful information. For each measurement (11 compounds, 20 repetitions), $\Delta \mathrm{R}, \mathrm{A}_{\text {Resp }}, \mathrm{A}_{\text {Recov }}$, and $\mathrm{A}_{\text {Resp }} / \mathrm{A}_{\text {Recov }}$ features were calculated to create a $4 \times 220$ feature vector as an input to the PCA generator. Each row represents a single measurement and each column represents a feature calculated for that particular measurement. To avoid overfitting, the dataset was divided into $60 \%$ training and $40 \%$ testing size. The abovementioned feature vectors were used as input to train the KNN, linear SVM, RF, and LDA classifiers using 10-fold CV for accuracy comparison. The overall classification accuracy for 11 compounds was noted above $90 \%$ for every classifier with an accuracy rate of 95\%, 95\%, $96 \%$, and 92\% for KNN, SVM, RF, and LDA, respectively (see Figure 13A). Additionally, most encountered misclassifications were analyzed through the confusion matrix [123]. Itoh et al. [130] reported highly selective VOC detection using different kinds of sensors in humid and pure air environments. The sensing array was composed of four commercially available semiconductor metal oxide sensors (TGS 2600, 2602, 2610, and 2620; Figaro Engineering Inc., Minoh, Japan); two semiconductor $\mathrm{Pt}, \mathrm{Pd}$, and $\mathrm{Au} / \mathrm{SnO}_{2}$ sensors; and two semiconductor $\mathrm{Zr}$-doped $\mathrm{CeO}_{2}$ sensors (bulk-type sensors). Response from all eight 
sensors to $1 \mathrm{ppm}$ of acetone was collected under a dry and humid air environment. It was found that bulk type sensors were not affected by the humidity ascribed to the different sensing mechanisms (see Figure 13B). The PCA results were obtained using response values from the sensors. First, normalized scores $\left(x_{t i}\right)$ were calculated using Equation (1) [130]:

$$
x_{t i}=\frac{\left(r_{t i}-\overline{r_{t}}\right)}{\sigma_{t}}
$$

where $t$ is the sensor index, $i$ is the sensor response analysis index, $r_{t i}$ is the sensor response analysis $i$ of sensor $t, \overline{r_{t}}$ is the average sensor response of sensor $t$, and $\sigma_{t}$ is the standard deviation of sensor $t$.

$$
|A-\lambda E|=0
$$

where $A$ is the matrix displayed in Equation (3) [130], $\lambda$ is the eigenvalue, and $E$ is the unit matrix.

$$
A=\left(\begin{array}{cccc}
C_{11} & C_{12} & \cdots & C_{1 m} \\
C_{21} & C_{22} & \cdots & C_{2 m} \\
\vdots & \vdots & \ddots & \vdots \\
C_{m 1} & C_{m 2} & \cdots & C_{m m}
\end{array}\right)
$$

where $C_{a b}$ is a correlation coefficient between sensors $a$ and $b\left(C_{11}=C_{22}=\cdots=C_{m m}=1\right)$ and $m$ is the maximum number of sensors. The eigenvalues can be acquired for each sensor index, as shown in Equation (4) [130].

$$
\lambda=\lambda_{1}, \lambda_{2}, \ldots \lambda_{m}\left(\lambda_{1}>\lambda_{2}>\ldots \lambda_{m}\right)
$$

where $C_{a b}$ is a correlation coefficient between sensors $a$ and $b\left(C_{11}=C_{22}=\cdots=C_{m m}=1\right)$, and $m$ is the maximum number of sensors. The eigenvalues can be acquired for each sensor index, as shown in Equation (4).

$$
A V_{j}=\lambda_{j} V_{j} \ldots \ldots \operatorname{Or}\left(A-\lambda_{j} E\right) V_{j}=0\left(\begin{array}{c}
v_{1 j} \\
v_{2 j} \\
\vdots \\
v_{m j}
\end{array}\right)
$$

Finally, the PCA $\left(Z_{j i}\right)$ was calculated by the product sum of the normalized score and eigenvectors, as shown in Equation (6) [130]:

$$
Z_{j i}=v_{1 j} x_{1 i}+v_{2 j} x_{2 i}+\cdots+v_{m j} x_{m i}
$$

Figure 13B shows the calculated PCA scores, confirming that bulk type sensors were not affected by the humidity. The PCA was performed for class discrimination. Classifiers were not trained for accuracy checks [130].

Jaeschke et al. [132] demonstrated an innovative e-nose system using a unique combination of analog and digital MOx sensors for ethanol and acetone detection in dry and humid environments. The sensing array consisted of 8 analog and 10 digital sensors. For PCA graphs, features and feature extraction methods were not described in their article. Nevertheless, they showed a good PCA graph. The entire dataset was divided into 75\% (300 measurements) for training and the remaining 25\% for testing (102 measurements). The hold-out CV method was used to train the LDA model. The maximum 76.4\% LDA accuracy was recorded for the classification of different VOC concentrations [132] (see Figure 14A). The lower accuracy rate might have been due to the inappropriate selection of the CV method since the hold-out method randomly shuffled the entire dataset for training and testing. Therefore, the probability of model training on all kinds of data examples might have been very low and may have caused the degradation in the model performance. 


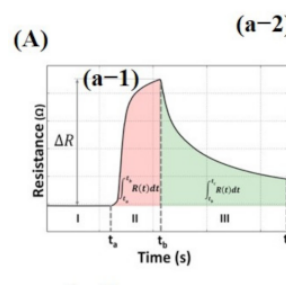

(a-3)

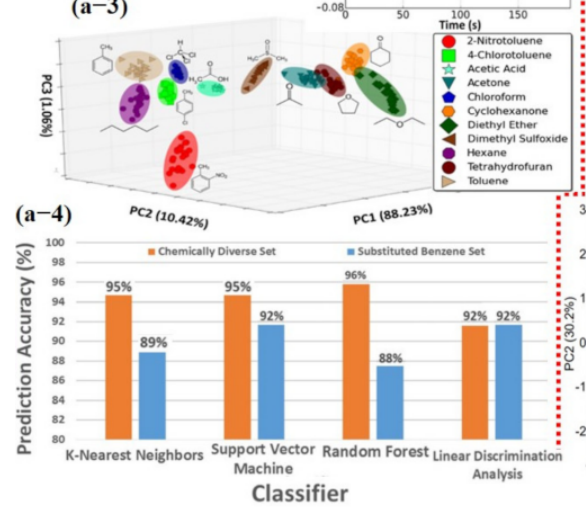

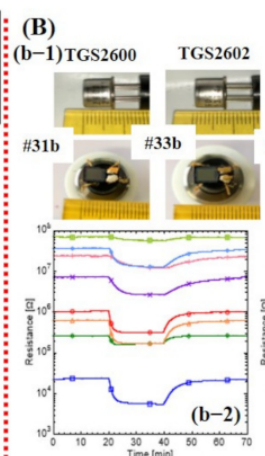
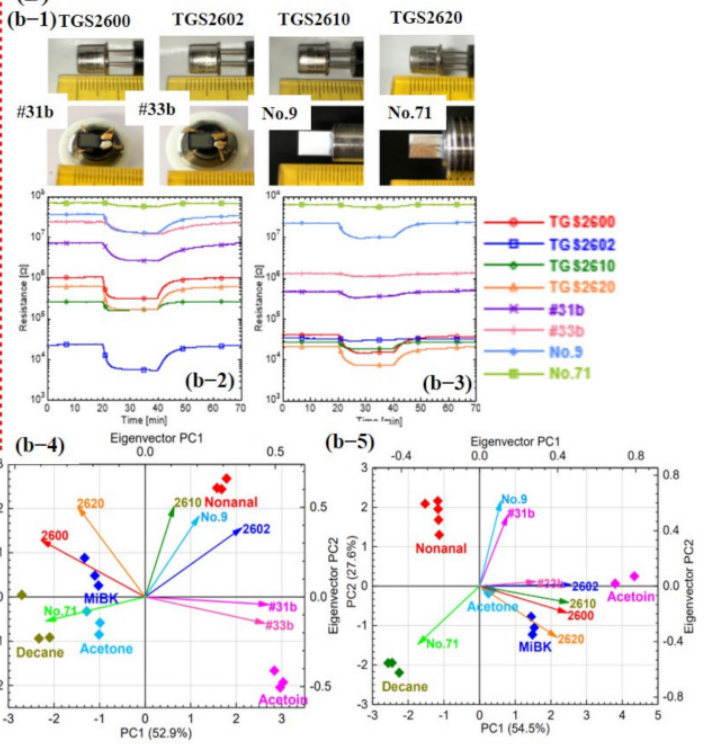

Figure 13. Smart sensing using machine learning: (A) Sensing of several compounds using single graphene-based chemiresistive sensor: (a-1) Typical response of chemiresistive sensor, regions I, baseline; II response time; and III, recovery. It indicates the potential features that can be extracted including the maximum change in resistance $(\Delta \mathrm{R})$, area of the response (red shaded area), and area of the recovery (green shaded area). (a-2) Normalized response for all compounds, ready for feature extraction. (a-3) PCA scores for the 11 compounds revealed small overlapping. (a-4) Different classifier prediction accuracy histogram, reproduced with permission from [123], copyright 2016 ACS. (B) e-nose system for smart detection of different compounds under dry and humid air: (b-1) Optical image for all the sensors. (b-2,3) Sensor response under dry and humid air, respectively. No. 9 and no. 71 sensors were not affected by humidity. (b-4,5) Corresponding PCA results of all sensor data in air and humid environment; PCA also confirmed the little humidity effect on no. 9 and no. 71 sensors. Reproduced with permission from [130], copyright 2017 MDPI.

In the same year, Tonezzer et al. [133] reported a single sensor (Pt-decorated $\mathrm{SnO}_{2}$ nanowires and pure $\mathrm{SnO}_{2} \mathrm{NWs}$ [134]) that is highly selective using thermal fingerprints. They checked the response of five different gasses, namely, ethanol, acetone, benzene, toluene, and $\mathrm{H}_{2}$, at different temperatures. The sensor response checked at different temperatures was then used as thermal fingerprints for feature extraction. With only one nanostructured material $\left(\mathrm{Pt}-\mathrm{SnO}_{2}\right)$ and five temperature values, their system was able to qualitatively and quantitatively discriminate all the gases with high accuracy [133] (see Figure 14B). The entire thermal fingerprint data were divided into $70 \%$ training (175 measurements) and 30\% testing (75 measurements) subsets and then were used to train the SVM model. Their trained model was able to differentiate among seven different gases and chemicals with an accuracy rate of $100 \%$. This was attributed to the selection of thermal fingerprints as feature input vectors for model training. However, the collection of input feature vectors at different operating temperatures for each gas/chemical may not be very feasible for sensor application in the real environment. 


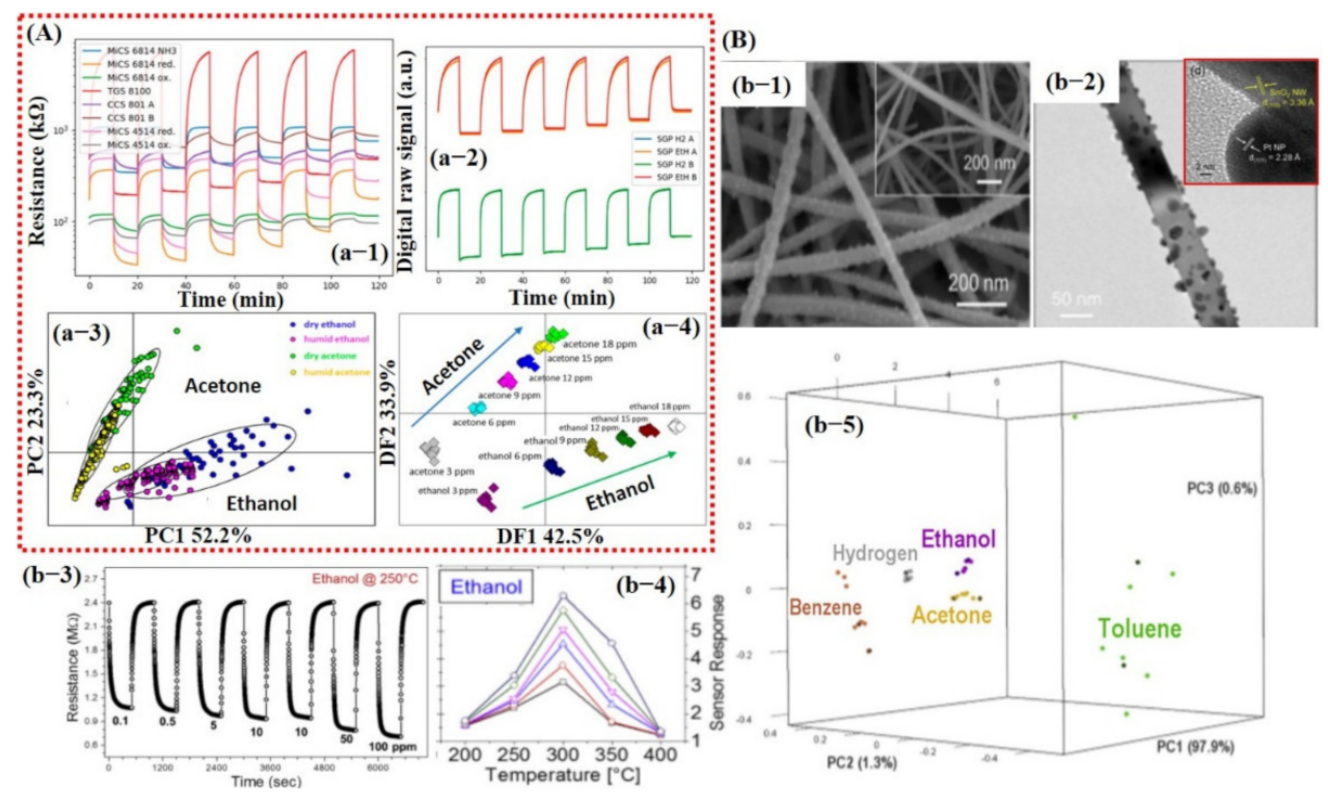

Figure 14. (A) e-nose system consisting of analog and digital sensors for detection of different compounds: (a-1) Raw data of analog sensors to acetone under humid conditions. (a-2) Raw data of digital sensors after exposure to acetone under humid conditions. (a-3) PCA score plot showing the effect of humidity. The measurements are labeled by condition and target compound. (a-4) LDA scores plot for the discrimination of compounds and concentrations of the measurements performed under dry and humid conditions. Reproduced with permission from [132], copyright 2019 ACS. (B) Smart sensing of different compounds using thermal fingerprints of a single sensor based on Pt NPs@SnO 2 NWs. (b-1) SEM image of Pt NPs decorated on $\mathrm{SnO}_{2} \mathrm{NWs}$. (b-2) Higher magnified TEM image of single $\mathrm{SnO}_{2} \mathrm{NW}$ decorated with Pt NPs. (b-3) Dynamic response during the exposure of different ethanol concentrations @ $250{ }^{\circ} \mathrm{C}$. (b-4) Sensor response to ethanol at different operating temperatures. (b-5) Three-dimensional plot of the principal components revealing excellent discrimination among different compounds. Reproduced with permission from [133], copyright 2019 Elsevier.

Besides chemicals and gas detection, chemiresistive-based sensors were also used to detect the quality of food using machine learning. For instance, Schroeder et al. [135] recently fabricated a chemiresistive sensing array using 20 different functionalized CNT sensors for classification of food quality using machine learning [135]. In order for one to build a sensing system that can differentiate between complex organic odor mixtures, the choice of sensors is critical. Various types of the functional groups were attached on the surface of CNTs, including transition metal complexes (S1, S2, S3, and S4) to bind organic acids and sulfur-containing compounds; ionic liquids (S5, S6, S7, and S8) to interact with ketones, aldehydes, alkanes, and aromatic compounds; porous polymers (S9, S10, S11, and S12) to detect a large number of organic vapors; cavitand and molecules (S13, S14, S15, S16, and S20) for detection of aromatic compounds and alcohols with size-exclusion properties; and metalloporphyrins (S17 and S18) to bind amines, alcohols, ketones, alkanes, and aromatic compounds [135]. For data processing to train a KNN model, features were directly collected from a specific window (120 s of exposure and $180 \mathrm{~s}$ of recovery) of the dynamic response, highlighted with a dotted line in Figure 15Aa-2. Later, tsfresh was also used to extract the features from the dynamic response, and 794 features were extracted, ranging from the coefficients of a CWT or FFT to parameters such as time series length, mean, max, and median, as well as many others. Featured random forest (f-RF) models were built on the tsfresh-extracted features, while the KNN models used the raw time series data (un-normalized) with the nearest neighbor of 1 supplying the class vote. The dataset was split into $80 \%$ training and $20 \%$ testing subsets. The f-RF 
(featured trained random forest) model was trained with 50 iterations, and after each iteration, the data were randomly shuffled. The accuracies from all 50 iterations were then averaged, and a standard deviation was calculated to determine overall model accuracy. The accuracies were calculated, and it was found that the f-RF model displayed maximum accuracy of $\approx 91 \%$ in predicting cheese with a specific set of sensor data (S4, S5, S6, and S20) (see Figure 15A). As expected from the dynamic response, feature-extracted PCA scores discriminated cambozola cheese, but some overlapping among other kinds of cheese was found. Moreover, the status of the tsfresh extracted was also checked, and 16 features were found with more useful information to accurately train the f-RF model (see Figure 15A). Among these 16 features, CWT and FFT were the most commonly occurring features. Therefore, it can be estimated that CWT and FFT are vital and contain much of the useful information for unique pattern generation [135]. Shekhar et al. [129] reported a CVDgrown graphene nanoribbon e-nose system consisting of 38 sensors for VOC detection. The schematic diagram, output response, and LDA accuracy graphs towards different VOCs are shown in Figure 16A. All 38 sensors showed the strongest response towards amines and alcohols. Therefore, amines and alcohol response datasets were used for model training. The response curves of various amines and alcohols were collected and normalized using Equations (7) and (8) [129] for their implementation as a feature vector to train an LDA model.

$$
\begin{gathered}
N=\frac{S_{i}}{S_{a v g}} \\
S_{a v g}=\frac{\sum_{k=1}^{n} S_{k}}{n}
\end{gathered}
$$

where $N$ is normalized data for model training, $S_{i}$ is response of $i^{\text {th }}$ sensor, $S_{\text {avg }}$ is the average response of all the sensors, and $n$ is the number of sensors. The LDA model trained on the normalized dataset showed $100 \%$ accuracy. However, the details on model training were not discussed. Recently, Acharyya et al. [126] presented VOC detection using single $\mathrm{SnO}_{2}$ hollow sphere sensors and machine learning. The sensor schematic diagram, response, and accuracy of different models are summarized in Figure 16B. The sensor responses at different operating temperatures (varying from 200 to $350{ }^{\circ} \mathrm{C}$ ), VOC concentration, response, response time, and recovery time were used as input features for model training. Different classifiers were studied, including RF, MLP, SVM, and NB, in order to develop the best fit model for the available input dataset. On the basis of these input feature values, the researchers allowed the algorithms to run using the 16 -fold CV technique in order to examine the accuracy for the different test datasets. Then, the results were averaged to produce final accuracy, which is defined as the ratio of the number of correct predictions to the total number of samples. The maximum accuracy was obtained around $85.93 \%$ for the RF model. The comparison in Table 1 shows various chemiresistive sensors/e-nose systems with a variety of extracted features and output accurate results. 


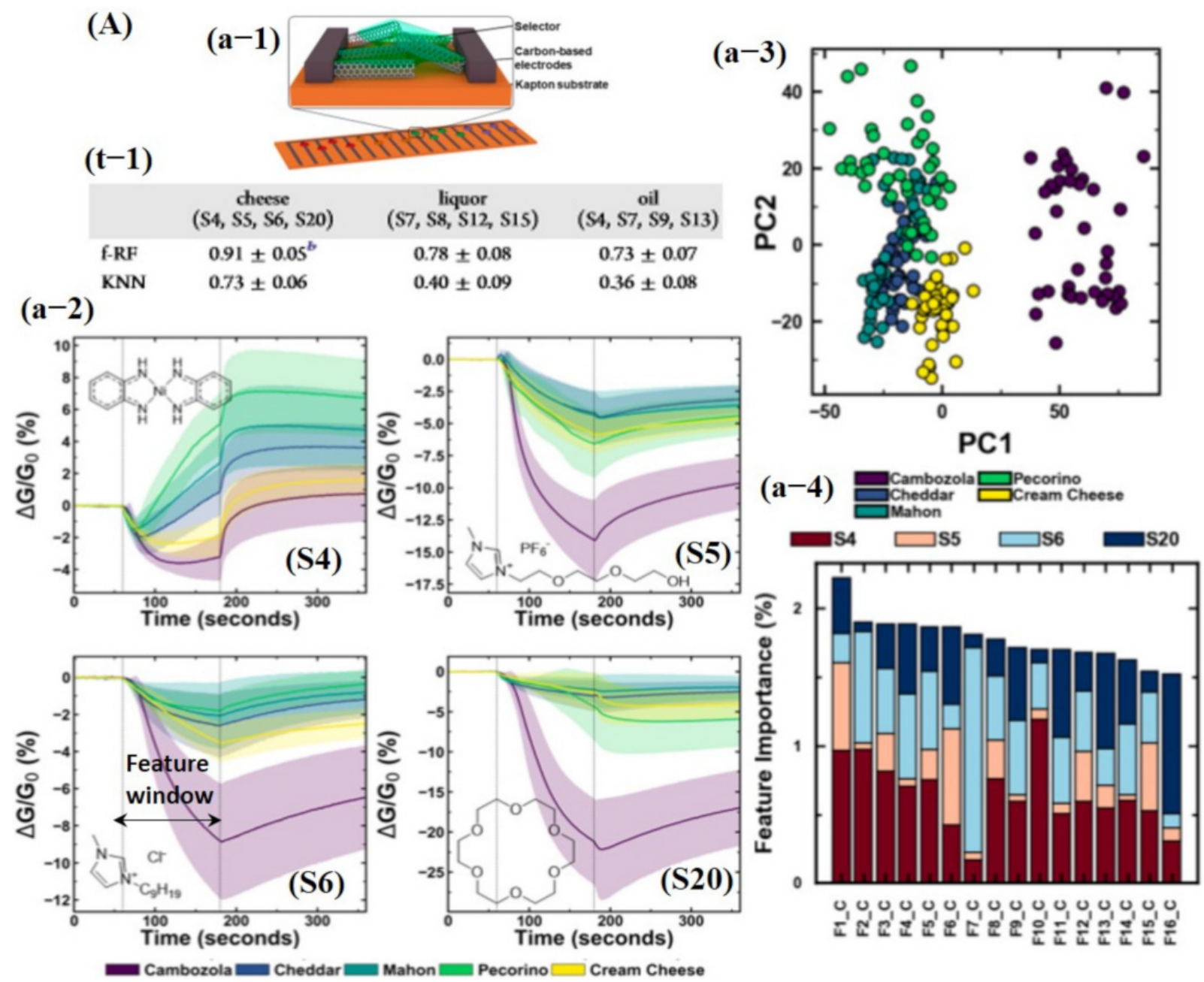

Figure 15. (A) Food classification using an array of 20 chemiresistive sensors and machine learning: (a-1) Schematic diagram of functionalized carbon nanotube (CNT) chemiresistive device. (a-2) Sensing response for S4, S5, S6, and S20 toward five kinds of cheeses. The response is represented as a change in conductance normalized to the conductance at the start of the exposure $(\Delta G / G 0)$. Each exposure started at $t=60 \mathrm{~s}$ and ends at $t=180 \mathrm{~s}$ (marked by dashed vertical lines used as features for KNN training). Each response was an average of 40 separate sensing experiments. The shaded area represents the standard deviation of the response. (a-3) PCA of extracted features from the five-cheese dataset showing the first two principal components. (a-4) Top 16 overall most important features in the five-cheese, wherein ( $t$ - 1 ) demonstrates the prediction accuracy toward different kinds of cheese using KNN and f-RF classifiers. Reproduced with permission from [135], copyright 2019 ACS. 


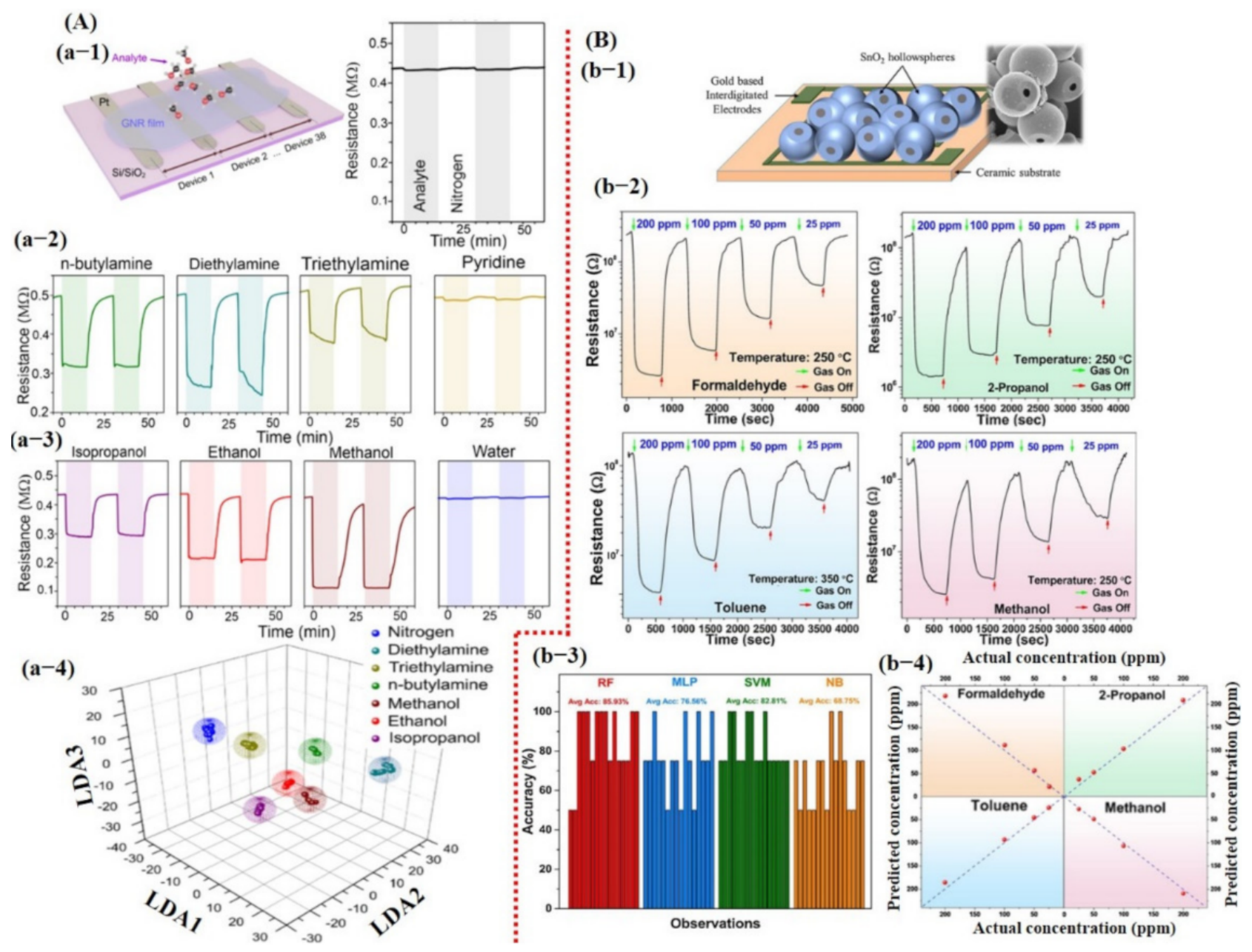

Figure 16. (A,B) summarize the detection results for different VOCs using chemiresistive devices and machine learning. (a-1) Schematic diagram of graphene nanoribbon sensors. (a-2) Real-time resistance change response toward different amines and alcohols. (a-3) 3D LDA graph representation with 100\% accuracy. Reproduced with permission from [129], copyright 2020 ACS. (b-1) Schematic diagram of fabricated hollow $\mathrm{SnO}_{2}$ sphere-based chemical sensor; inset presents the SEM image of synthesized hollow spheres. (b-2) Real-time resistance change response to different chemicals at various concentration levels. (b-3) Average accuracy bar graph for each model. (b-4) Representation of concentration prediction for each chemical. Reproduced with permission from [126], copyright 2020 Elsevier. 
Table 1. A comparison of some of the reported extracted features and output accuracies for chemiresistive devices.

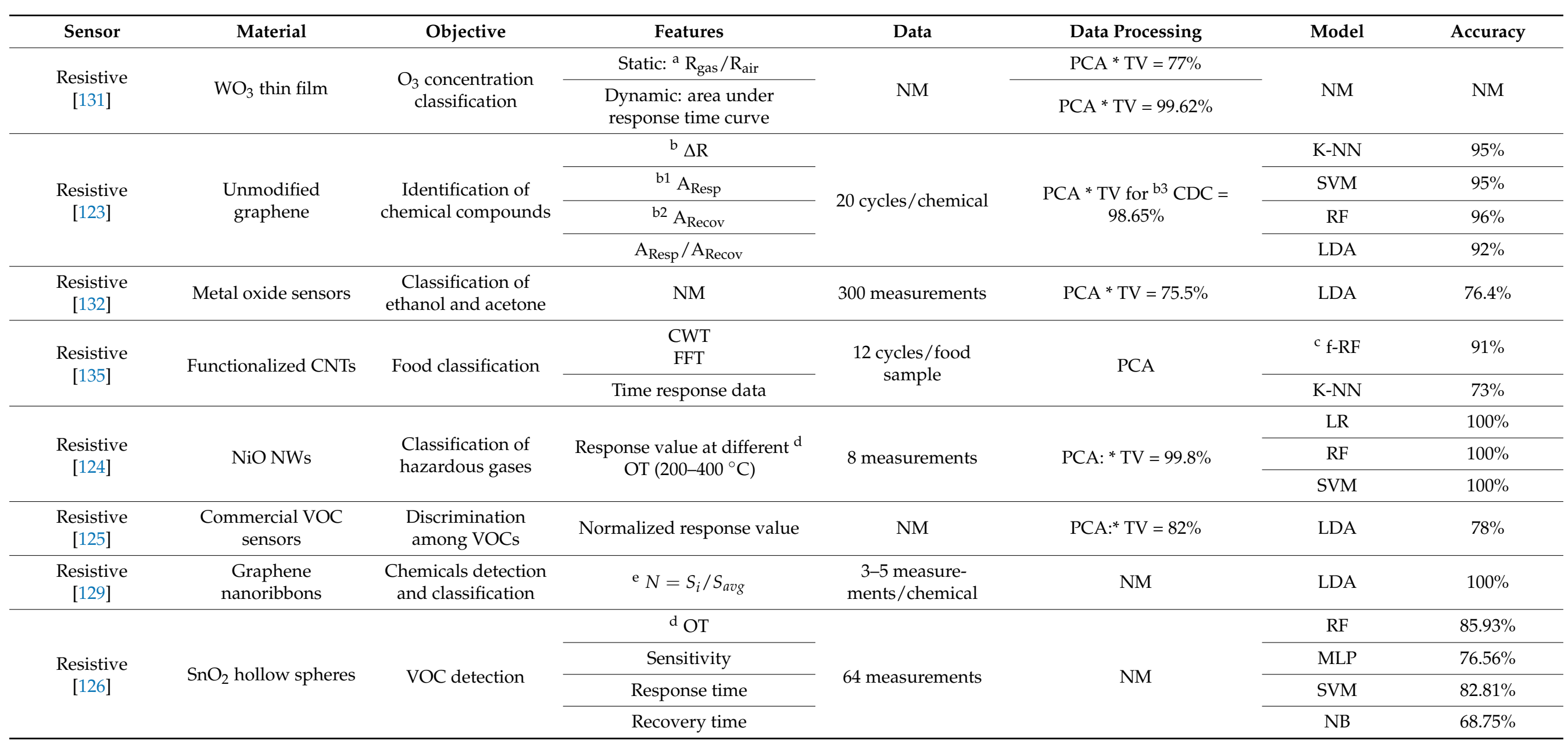

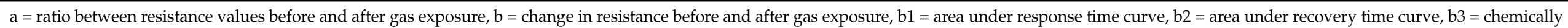

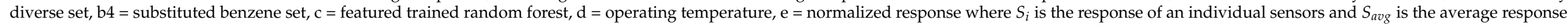
across the array of $n$ sensors, ${ }^{*}=$ total variance, $\mathrm{NM}=$ not mentioned. 


\subsection{Field Effect Transistor-Based Smart Gas Sensors Using Machine Learning}

Field-effect transistors (FETs) have also been widely studied for gas/chemical sensing due to their small size and trace level molecule detection with high sensitivity. A FET device consists of three terminals: drain (D), source (S), and gate (G). The current flows from the drain to the source $\left(\mathrm{I}_{\mathrm{DS}}\right)$ can be controlled by applying a voltage load at the gate [136]. The sensing materials of FETs are usually based on thin films or monolayers (graphene, TMDCs, and semiconductors) functionalized/decorated with ligands and catalysts [136]. In comparison with resistive type sensors, they are more complex and expensive to fabricate. A typical FET sensing device shows a change in the IDS curve upon exposure to a target molecule within a particular range of gate voltage $\left(\mathrm{V}_{\mathrm{g}}\right)$. Similar to resistive-type sensors, selective sensing material, useful feature extraction, and proper model training is vital to developing a smart FET sensor with the ability to accurately discriminate among various gases. In 2014, Wang et al. [137] reported functionalized single silicon (Si) nanowire (NW) for accurate detection of 11 VOCs using artificial neural networks (ANN). Features were extracted from the original and logarithmic $\mathrm{I}_{\mathrm{DS}}$ curve after gas exposure, with $V_{g}$ ranging from 40 to $-40 \mathrm{~V}$. The threshold voltage $\left(V_{\text {th }}\right)$, hole mobility, $\left(\mu_{\mathrm{h}}\right)$, and $\mathrm{I}_{\mathrm{on}}$ (defined as $\mathrm{I}_{\mathrm{DS}} @ \mathrm{~V}_{\mathrm{g}}=-40 \mathrm{~V}$ ) were obtained and calculated from the original IDS curve, while subthreshold swing (SS) was acquired from logarithmic IDS curve and was used as input features for a neural network. A response curve is shown in Figure $17 \mathrm{~A}$, which is labeled with extracted features and the variation graphs for the features upon exposure to different VOCs. Their trained ANN was perfectly able to recognize 11 VOCs and their binary/ternary combinations as well [137]. Similarly, Guo et al. [138] developed an ANN flexible gas sensor based on ultra-large area $\mathrm{MoSe}_{2}$ nanosheet. They proposed a machine learning and data-driven approach to predict the location of the gas source at home in a macroscopic scene. The process consisted of four layers, an input layer, two hidden layers, and an output layer. The input layer is the data array acquired from the sensors, while the output layer is the location of the gas source. Their proposed approach achieved a satisfactory prediction accuracy for $\mathrm{NO}_{2}$ and $\mathrm{NH}_{3}$ [138].

In 2019, Bian et al. [139] synthesized a sensing array using different metal catalysts decorated on single-walled carbon nanotube (SWCNTs) to develop a FET device for the detection of purine compounds (adenine, guanine, xanthine, uric acid, and caffeine). The 11 different features were extracted from the response curve of the FET device. The response curve labeled with extracted features is shown in Figure 17Bb-2. For training, the entire dataset was split into 10 subsets, with each subset containing the ratio of caffeine to non-caffeine of 1:2.54. Nine subsets were used to build the model, and one subset was used for testing the robustness of the model. The stratified 10-fold cross-validation was used to train the SVM model. The stratified k-fold CV is an efficient approach to shuffle the entire dataset and then divide it into equal subsets with a good representation of all the training examples. Therefore, their trained SVM model was successfully able to distinguish caffeine with an accuracy rate of $93.4 \%$ [139] (see Figure 17Bb-3). 


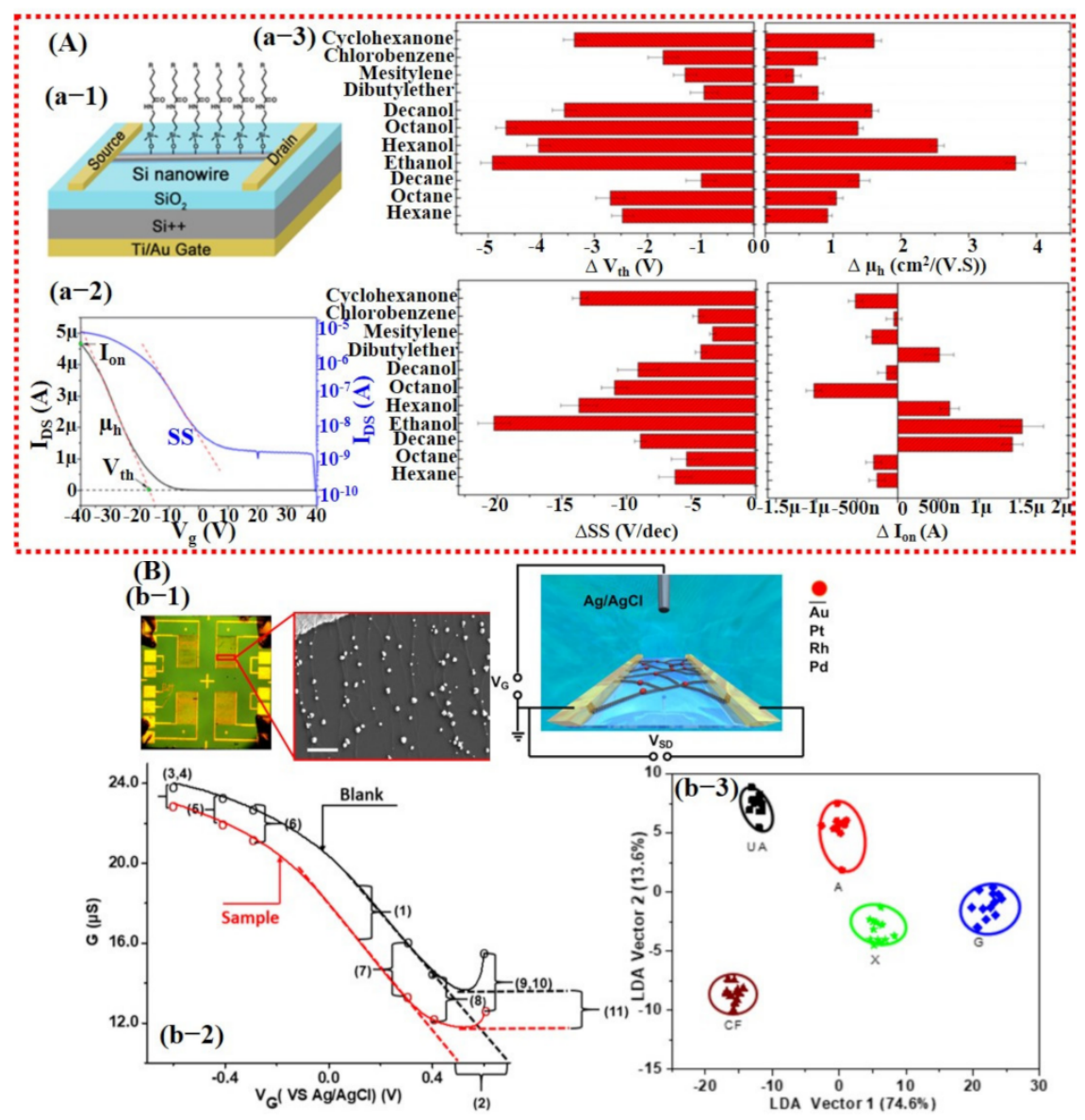

Figure 17. Smart gas sensing using FET-based devices and machine learning. (A) Modified single Si NW-based FET device for VOCs detection: (a-1) Schematic diagram of the fabricated FET device. (a-2) Typical IDS response curve of FET device labeled with the extracted features $\left(\mathrm{V}_{\mathrm{th}}\right)$, ion, hole mobility, and SS for data processing. (a-3) Variations in (a) $V_{t h},(b) \mu_{h}$, (c) SS, and (d) ion of sensor S4 upon exposure to various VOCs at a concentration of $\mathrm{pa} / \mathrm{po}=0.08$. Reproduced with permission from [137], copyright 2014 ACS. (B) Metal catalyst decorated on the CNT-based FET device for detection of purine compounds: (b-1) Optical and schematic images of the fabricated device. The inset reveals the SEM image of CNTs decorated with NPs. (b-2) Pt NP-decorated NTFET response with and without caffeine $(1 \mathrm{mM})$ solutions. Selected features (11) were calculated from the NTFET curves before and after exposure of caffeine solutions. (b-3) LDA plots for purine compound discrimination. Reproduced with permission from [139], copyright 2019 ACS.

Most recently, Hayasaka et al. [140] fabricated a highly selective sensor using pristine graphene and $\mathrm{ALD}-\mathrm{RuO}_{2}$-based GFET devices with machine learning. In their proposed scheme, the measured V-shaped conductivity profiles were decoupled into four distinctive physical properties combined with other parameters. These four parameters were used as input feature vectors to classify different gases including electron mobility $\left(\mu_{e}\right)$, hole mobility $\left(\mu_{h}\right)$, ratio of the electron and hole concentration $\left(n_{e / h}\right)$, the ratio of the residual carrier, and charge impurity concentration $\left(n^{*} / n_{i m p}\right)$, represented in Equations (9)-(12), respectively [140].

$$
\begin{aligned}
\mu_{e} & =\frac{1}{C_{G}} \frac{\Delta \sigma_{e}}{\Delta V_{G}} \\
n_{e} / h & =\frac{C_{G}}{e}\left|V_{N P}\right| \\
\mu_{h} & =\frac{1}{C_{G}} \frac{\left|\Delta \sigma_{h}\right|}{\Delta V_{G}}
\end{aligned}
$$




$$
\frac{n^{*}}{n_{i m p}}=\frac{1}{20} \frac{h}{e^{2}} \sigma_{0}
$$

where $C_{G}$ is the gate capacitance per unit area, $\Delta \sigma_{e}$ is the change in electron conductivity, $\Delta \sigma_{h}$ is the change in hole conductivity, $\Delta V_{G}$ is the change in gate voltage, $n_{h}$ is the hole concentration, $e$ is the elementary charge, $V_{G}$ is the gate voltage, $V_{N P}$ is the gate voltage at the neutrality point (NP), $h$ is Planck's constant, and $\sigma_{0}$ is the minimum conductivity at the NP. The electron mobility and hole mobility turned out to be the most important features, with electron mobility having much of the information of the data. The confusion matrix displayed $100 \%$ correct values for ALD-RuO 2 -GFET, while pure graphene GFET confused some values with others [140] (see Figure 18).

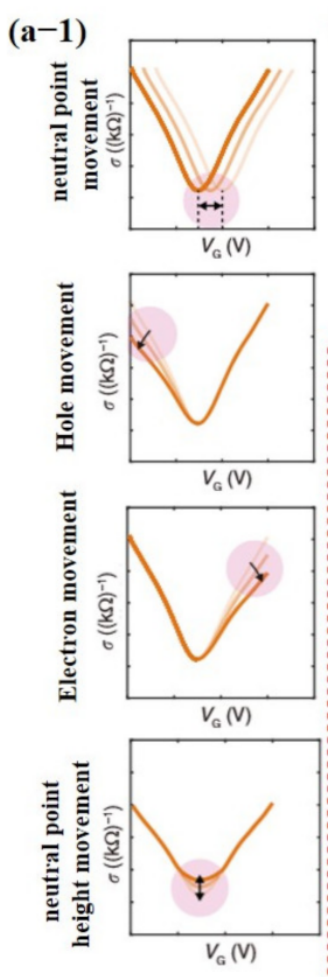

$(\mathbf{a}-2)$
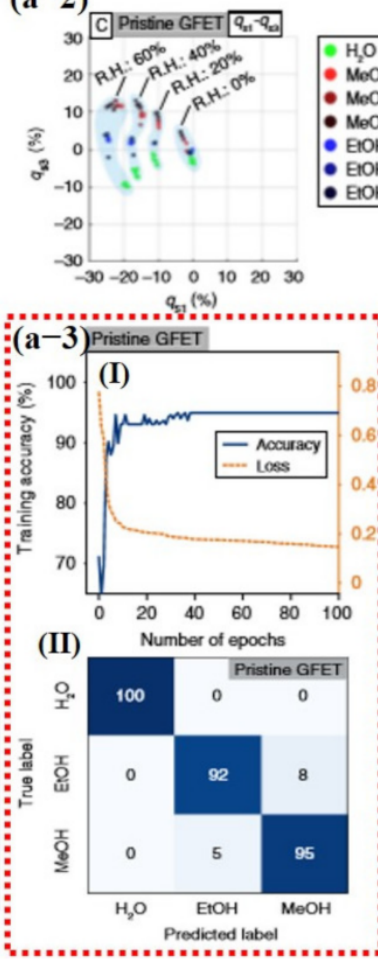

$(\mathbf{a}-5)$
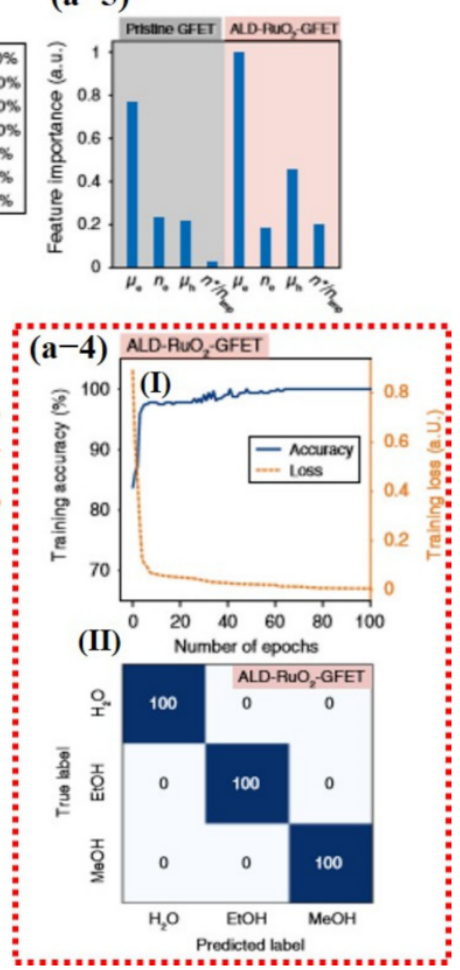

Figure 18. Pristine graphene and $\mathrm{AL}-\mathrm{RUO}_{2}$-based GFET devices for detection of different compounds under dry and humid environments. (a-1) Schematic illustration of extracted features for data processing, movement of the charge-neutral point $(\mathrm{Np})$, variation in hole branch, variation in electron branch, and variation in height of the charge Np. (a-2) 3D gas-sensing patterns of the binary gas mixtures projected onto a representative $2 \mathrm{D}$ plot. The gas-sensing patterns are grouped by light blue colored regions, and the corresponding background relative humidity (R.H) levels are labeled. (a-3 (I)) Training accuracy and the training loss history from results using the pristine graphene; (II) confusion matrix of the pristine GFET. (a-4 (I)) Training accuracy and the training loss from results using the ALD-RuO ${ }_{2}$ GFET; (II) confusion matrix of the ALD- $\mathrm{RuO}_{2}$ GFET with $100 \%$ true values. (a-5) Normalized feature importance for the eight tested features. Reproduced with permission from [140], copyright 2020 Springer Nature.

A multilayer perceptron classifier with a feed-forward neural network architecture was implemented and trained using the 4D input feature vector of the two GFETs (pure graphene and $\mathrm{ALD}-\mathrm{RuO}_{2}$ ). To avoid overfitting, the entire dataset was randomly shuffled in several ways and then separated via a stratified split, where $20 \%$ was reserved as the testing set and the remainder constituted the training set. The neural network model was trained for 40 epochs to obtain the maximum accuracy, and the time required for 40 epochs was $0.0519 \mathrm{~s}$. The accuracies of the pristine GFET device and ALD-RuO $2-$ GFET device 
were $96.2 \%$ and $100 \%$, respectively. The cross-validation results indicated that the pristine GFET device had a mean accuracy of $95.4 \%$ and a standard deviation of $2.5 \%$, whereas the ALD- $\mathrm{RuO}_{2}$-GFET device had a mean accuracy of $99.6 \%$ and a standard deviation of $0.8 \%$. [140]. A comparison of FET devices with extracted features and output accuracies is summarized in Table 2.

Table 2. A comparison of some of the reported extracted features and output accuracies for FET devices.

\begin{tabular}{|c|c|c|c|c|c|c|}
\hline Sensor & Material & Objective & Features & Data & Model & Accuracy \\
\hline \multirow{3}{*}{$\begin{array}{l}\text { FET } \\
{[141]}\end{array}$} & \multirow{3}{*}{$\begin{array}{l}\text { Functionalized } \\
\text { Si NWs }\end{array}$} & \multirow{3}{*}{ Cancer diagnosis } & $\mathrm{V}_{\text {th }}$ & \multirow{3}{*}{$\begin{array}{l}\text { Data from } 77 \\
\text { volunteers }\end{array}$} & \multirow{3}{*}{ DFA } & \multirow{3}{*}{$85 \%$} \\
\hline & & & $\mu_{\mathrm{h}}$ & & & \\
\hline & & & $\mathrm{I}_{\mathrm{DS}} @ \mathrm{~V}_{\mathrm{GS}}=0$ & & & \\
\hline \multirow{3}{*}{$\begin{array}{l}{ }^{\text {a }} \text { CNFET } \\
\text { [139] }\end{array}$} & \multirow{3}{*}{ Metal/CNTs } & \multirow{3}{*}{ Purine classification } & Slop variance & \multirow{3}{*}{$\begin{array}{l}12 \text { measure- } \\
\text { ments/chemical }\end{array}$} & \multirow{3}{*}{ SVM } & \multirow{3}{*}{$93.4 \%$} \\
\hline & & & $V_{\text {th }}$ & & & \\
\hline & & & $\begin{array}{l}\text { Changes in conductance } \\
\text { at various } V_{G} \text { values }\end{array}$ & & & \\
\hline \multirow{2}{*}{$\begin{array}{l}\text { b ISFET } \\
\text { [142] }\end{array}$} & \multirow{2}{*}{$\mathrm{HfO}_{2}$ layer } & \multirow{2}{*}{$\begin{array}{l}\mathrm{pH} \text { and light } \\
\text { intensity }\end{array}$} & \multirow{2}{*}{$\begin{array}{l}\text { Normalized response at } \\
\text { various biasing voltages }\end{array}$} & \multirow{2}{*}{$\begin{array}{c}1920 \\
\text { measurements } \\
\text { from } 32 \text { sensors }\end{array}$} & SVM & $100 \%$ \\
\hline & & & & & ${ }^{\mathrm{b} 1} \mathrm{BPNN}$ & $100 \%$ \\
\hline \multirow{4}{*}{$\begin{array}{l}\text { FET } \\
{[140]}\end{array}$} & \multirow{2}{*}{${ }^{\mathrm{c}}$ GFET } & \multirow{4}{*}{$\begin{array}{l}\text { Water, methanol, and } \\
\text { ethanol detection }\end{array}$} & $\mu_{e}$ & \multirow{4}{*}{$\begin{array}{l}100 \text { measure- } \\
\text { ments/chemical }\end{array}$} & \multirow{4}{*}{$\begin{array}{l}\text { Multi-layered } \\
\text { ANN }\end{array}$} & \multirow{2}{*}{$96.2 \%$} \\
\hline & & & $n_{e / h}$ & & & \\
\hline & \multirow{2}{*}{$\begin{array}{c}{ }^{c 1} \text { ALD- } \\
\mathrm{RuO}_{2} \text {-GFET }\end{array}$} & & $\mu_{h}$ & & & \multirow[b]{2}{*}{$100 \%$} \\
\hline & & & $\frac{n^{*}}{n_{i m p}}$ & & & \\
\hline
\end{tabular}

$\mathrm{a}=$ carbon nanotube field effect transistor, $\mathrm{b}=$ ion-sensitive field effect transistor, $\mathrm{b} 1$ = back propagation neural network, $\mathrm{c}=$ graphene field effect transistor, $\mathrm{c} 1$ = atomic layer deposition-grown $\mathrm{RuO}_{2}$ thin film field effect transistor.

\subsection{Smart Breath Analyzers Using Machine Learning}

A multilayer perceptron classifier with a feed-forward neural network architecture was implemented and trained using the 4D input feature vector of the two GFETs (pure graphene and $\mathrm{ALD}-\mathrm{RuO}_{2}$ ). To avoid overfitting, the entire dataset was randomly shuffled in several ways and then separated via awareness of personal health conditions demanding the development of safe, easy, and noninvasive disease diagnostic tools with great accuracy. Analysis of various VOCs concentration levels in exhaled breath samples opens up a new frontier in the medical sector due to its easy installation, cheap fabrication, and noninvasive diagnostic nature. However, the development of a small size breath analyzer with accurate and fast detection of a particular compound at a certain concentration level among 1000 VOCs is still a great challenge [143-145]. Enormous efforts have been made for the fabrication of a smart, small-size, economical, highly selective, and accurate breath analyzer using both chemiresistive and FET mechanisms. In fulfillment of such requirements, Haick et al.'s [141,146-148] research group made significant efforts and published a series of research articles using ligand-functionalized Au NPs. In 2009, lung cancer detection in exhaled breath was performed using functionalized Au NPs [146]. They built a sensing array using a surface modification of Au NPs (5 nm) with several organic ligands including dodecanethiol, decanethiol, 1-butanethiol, 2-ethylhexanethiol, hexanethiol, tert-dodecanethiol, 4-methoxy-toluenethiol, 2-mercaptobenzoxazole, and 11-mercapto-1-undecanol. Interestingly, sensors functionalized with 4-methoxy-toluenethiol, and 2-mercaptobenzoxazole/11mercapto-1-undecanol showed detection limits of 2-10 ppb on exposure to acetaldehyde (a promising VOC for lung cancer) and formaldehyde (a promising VOC for breast cancer). Furthermore, 2-mercaptobenzoxazole-Au NPs (red diamonds) and tert-dodecanethiol-Au NPs (black triangles) displayed a significant difference in responses towards healthy and lung cancer patients, suggesting them as a promising candidate for the detection of lung cancer [146] (see Figure 19A). The response data obtained from healthy and lung cancer breath samples were then processed through PCA for classification. The PCA graph shown 
in Figure 19A demonstrates highly discriminated results without any overlapping. Features for PCA analysis and training of any classifier were not discussed in their article [146]. Likewise, in 2010, the same group developed ligand-modified Au NP sensing array for the detection of lung, breast, colorectal [147], and prostate cancers from exhaled breath. Their PCA graph illustrates a discriminative signature pattern for each kind of cancer disease with no overlapping on healthy samples. They mentioned that this is an attempt for the development of a cost-effective, easy-to-use, portable, and non-invasive diagnostic tool for detecting lung, breast, colorectal, and prostate cancers through a single breath test [147]. In 2015, a surface-modified Si NW-based FET sensor was developed for the diagnosis of gastric cancer. Instead of sensing arrays, they developed a single sensor-based FET device to selectively detect gastric cancer-related VOCs [141]. For data processing, three features were extracted from the $\mathrm{I}_{\mathrm{DS}}$ vs. $\mathrm{V}_{\mathrm{GS}}$ curves as a function of the exposure time towards the targeted VOCs: the threshold voltage $\left(\mathrm{V}_{\mathrm{th}}\right)$; hole mobility $\left(\mu_{\mathrm{h}}\right)$, inferred from the linear part of the curve; and the current at zero applied gate voltage $\left(\mathrm{I}_{\mathrm{DS}} @ \mathrm{~V}_{\mathrm{GS}}\right.$ $=0$ ), as a representative subthreshold current (see Figure 20A) [141]. The dataset of each analysis was divided into training and validation sets. A total of $75 \%$ of each group was selected randomly for the training set, and $25 \%$ of each group were left out for testing. Leave-one-out CV was conducted to train the DFA for the classification of the number of true-positive, true-negative, false-positive, and false-negative predictions. The training set using only one sensor (S1) showed $87 \%$ sensitivity, $81 \%$ specificity, and $83 \%$ accuracy [141]. Kahen et al. [148] developed ligand-functionalized Au NP sensing array on a flexible substrate for diagnosis of ovarian carcinoma from exhaled breath. In order to generate distinctive responses from each target compound and to increase the number of features for data processing along with resistance change, they also observed the bending state of the substrate upon absorption of the particular compound. The extracted features from the response curve are shown in Figure 19B. Discriminant factor analysis (DFA) was used with the leave-one-out CV method to find the sensitivity, specificity, and accuracy of each sensor using bending-related features from only one sensor. Their DFA result showed 83.4\% sensitivity, $80.8 \%$ specificity, and $81.8 \%$ accuracy, which was comparable with previously published results [148] (see Figure 19B).

In 2018, Sujono et al. [150] reported an e-nose for asthma diagnosis in exhaled breath that can predict the related VOC detection accuracy using SVM. The e-nose consists of seven commercial sensors including $\mathrm{CO}, \mathrm{H}_{2}, \mathrm{NOx}, \mathrm{H}_{2} \mathrm{~S}, \mathrm{NH}_{3}, \mathrm{VOCs}$, and $\mathrm{CO}_{2}$. A window of dynamic response curve (30 to $49 \mathrm{~s}$ ) was used as a feature to train the SVM classifier. Their system can successfully distinguish between healthy and asthma subjects with an accuracy rate of $89.5 \%$ [150].

In the same year, Park et al. [149] demonstrated an ionic liquid-functionalized carbon nanotubes (CNTs) sensing array for detection of exhaled breath-related VOCs. They developed a new platform for the selective and sensitive detection of VOCs by exploring the influence of cation and anion and identifying swelling as one of the sensing mechanisms. Nine sensors were built by surface modification of CNTs for the detection of 3-heptanone, heptanal, 2-methylpentane, benzene, and toluene. Figure 20B shows the PCA scores and it indicates the excellent discriminating ability of the sensors among different VOCs. However, the extracted features for obtaining PCA graphs were not discussed in their work. Ionic liquid demonstrates promising capabilities in detecting several VOCs, with distinguished transient patterns eventually leading to the development of highly selective VOC sensors using machine learning [149]. 


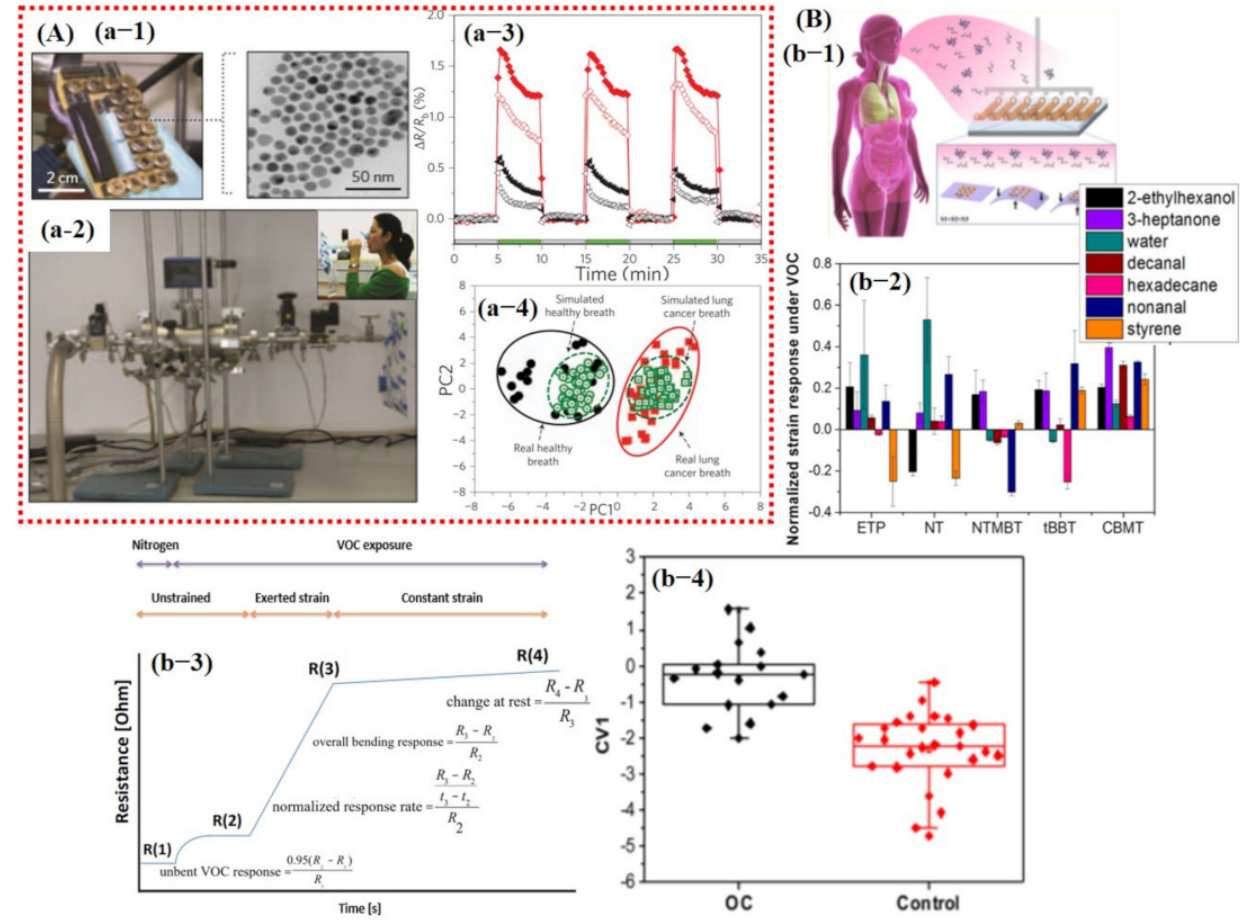

Figure 19. Smart breath analyzers using chemiresistive devices and machine learning. (A) Lung cancer detection using functionalized Au NPs from exhaled breath: (a-1) Optical image of sensor array; SEM image of Au NPs. (a-2) Breath sample collection and sensing setup. (a-3) Functionalized $\mathrm{Au}$ NPs sensor response towards healthy (filled symbol) and lung cancers patients (empty symbol). The significant change in response can be seen from the graph. (a-4) PCA of the dataset of real and simulated breath. Reproduced with permission from [146], copyright 2020 Nature. (B) Functionalized $\mathrm{Au}$ NPs onto flexible substrate was used to detect the ovarian carcinoma from exhaled breath. (b-1) Schematic diagram of fabricated sensor device with bending illustration upon exposure of any VOC. (b-2) Normalized strain response produced in different ligands under various VOCs. (b-3) Extracted feature graph for data processing. (b-4) Separation of the OC-positive and control groups (OC = ovarian cancer) using LDA. Reproduced with permission from [148], copyright 2015 ACS.

Although machine learning is treated as one of the major contributors in building smarter and selective sensors, there are still great obstacles in terms of developing economical, miniaturized, low-powered smarter sensors with the ability to accurately discriminate among different gas classes and concentration levels under various environmental circumstances (dry and humid conditions). Most of the metal oxide gas sensors show sensitivity to different gases at different concentrations, with minute change in their response curve. Therefore, it is essential to accurately detect and categorize the class of gas with its concentration level through proper machine learning algorithms in order to avoid any unwanted false positives. Further improvements in different aspects are required for successful implementation in real-world application; for instance, the development of a sensor with a low aging effect that works properly under different environmental conditions with good repeatability, and the implementation of different data processing algorithms to accurately train a model with minimum possible dataset examples and low power consumption. In the early days, larger datasets under all circumstances were required with maximum possible examples to enhance the training probability rate of the final statistical model. Several e-nose systems with multiple arrays of sensors were utilized to obtain the large datasets with all sensing examples. Recently, this problem has been partially resolved by introducing the k-fold cross-validation technique in which the model is trained and tested on all available examples; thus, smaller datasets can be used to accurately train a model. However, in this case, the signal processing unit may consume higher power due 
to the large number of iterations for the model training. The power consumption may be compensated through the sensory unit by reducing the arrays of sensors to a single sensor. Machine learning is successfully able to selectively detect various chemical compounds using a single sensing device (low-power consumption) with the k-fold cross-validation (smaller datasets) technique. However, the complex, expensive, and large hardware setups of smart sensors are pressing the need for developing nano/micro-sized sensory and signal processing units to accomplish the whole machine learning process on a single MEMS/NEMS chip for the miniature and portable smart system.
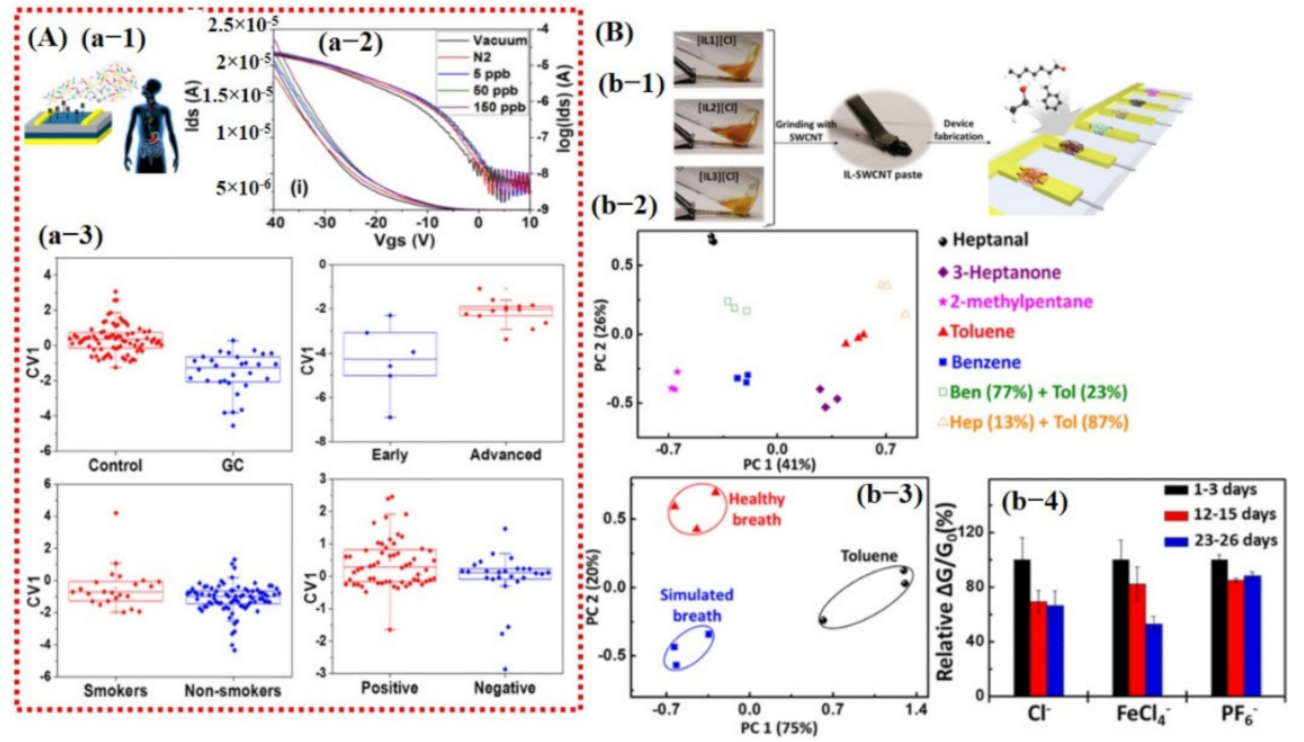

Figure 20. Smart breath analyzers using FET devices and machine learning: (A) Surface-modified single Si NW FET device for cancer detection: (a-1) Schematic diagram of fabricated sensor. (a2) Variation in IDS curve upon exposure to the increasing concentrations of VOCs. (a-3) CV1 values resulting from DFA analysis of the breath samples gastric cancer vs. control, early stages vs. advanced stages, smokers vs. nonsmokers, H. pylori-positive vs. H. pylori-negative. Reproduced with permission from [141], copyright 2015 ACS. (B) Ionic liquid-functionalized CNT-based FET sensing array was used for detection of different VOCs: (b-1) Schematic diagram of a fabricated array. (b-2,3) PCA results towards different VOCs. (b-4) The stability of ionic liquid after several days. Reproduced with permission from [149], copyright 2018 ACS.

\section{Conclusions and Outlooks}

This review covers the recent state-of-the-art advancement in the field of gas sensing using different techniques and their limitations and solutions using machine learning tools. The article is divided into two parts: The first part emphasizes the recent progress in the field of gas sensing, their different performance improvement methods, and current challenges. The second part highlights the significance of machine learning as a potential approach to tackle these limitations. Moreover, recent development in smart sensors and breath analyzers using machine learning has been also discussed in details.

For the implementation of a sensor in a real-world application, a sensor must possess several essential sensing performance features including excellent sensitivity, fast response/recovery time, repeatability, long-term stability (in humid and high-temperature environments), and selectivity. Different performance-enhancing methods have been discussed, such as composition/hybridization, doping of heteroatom, $\mathrm{p}-\mathrm{n}$ junction formation, and core-shell structures. According to the base material, we categorized gas sensors into three types: (1) graphene-based, (2) TMDC-based, and (3) semiconductor/metal oxidebased. Their computational studies were also covered to realize the major performanceenhancing factors. Furthermore, performance improvement methods for all three categories 
with their advantages and drawbacks were detailed. It was concluded that performanceenhancing methods certainly improve sensitivity, response/recovery time, repeatability, the limit of detection, and operating temperature. However, excellent selectivity and long-term stability in different environments are still great challenges. Therefore, it is suggested that with the development of novel material, efficient data processing techniques using machine learning are greatly needed in order to tackle selectivity and long-term stability.

Machine learning has been extensively used in building highly selective smart gas sensors and breath analyzers. Data processing is a major contributor since the success of the machine learning process relayed on it. It aims to extract robust feature information from the dynamic sensor response, which can represent the unique "fingerprint" patterns for a particular gas to ensure the effectiveness of the subsequent pattern recognition algorithm. This article discussed the state of the art of chemiresistive and FET-based smart gas sensors along with the most important features, which can be derived from the original dynamic response. Moreover, recent evolution in breath analyzers using machine learning has been also covered in this article.

Machine learning shows great potential in solving critical issues related to chemical gas sensors and plays a significant role in building smart sensors with improved sensing abilities, especially selectivity and long-term stability. However, they are complex, expensive, consume high power, and require a large hardware setup for their implementation in a realistic application. This presses the need for developing nano/micro-sensors along with signal processing and machine learning algorithm units on a single flexible substrate using MEMS/NEMS technology for the realization of miniature and portable electronics/electrical revolutionary smart world.

Author Contributions: U.Y.: Collected/arranged the material and write the article, M.I.Y.: Proposed the topic, supervised and revised the article. All authors have read and agreed to the published version of the manuscript.

Funding: This publication is based upon work supported by King Abdullah University of Science and Technology (KAUST).

Conflicts of Interest: The authors declare no conflict of interest.

\section{Appendix A}

Here, we present some background information on the PCA technique. PCA aims to reduce redundancy in a large dataset through diagonalization of the correlation matrix of the original dataset for the identification of the most important parameters in defining the actual degree of freedom or response of a system. The variance and covariance among the data values can be obtained by taking the dot product of the dataset with its transpose, as shown in Equation (A1)

$$
C_{X}=\frac{1}{n-1} X X^{T}
$$

where $C_{X}$ is the correlation matrix of dataset $X$ and $n$ is the number of variables.

$$
X=\left(\begin{array}{c}
\vec{x}_{a} \\
\vec{y}_{a} \\
\vec{x}_{b} \\
\vec{y}_{b} \\
\vec{x}_{c} \\
\vec{y}_{c}
\end{array}\right)
$$




$$
C_{X}=\left(\begin{array}{cccccc}
V_{x_{a} x_{a}} & C V_{x_{a} y_{a}} & C V_{x_{a} x_{b}} & C V_{x_{a} y_{b}} & C V_{x_{a} x_{c}} & C V_{x_{a} y_{c}} \\
C V_{y_{a a x}} & V_{y_{a} y_{a}} & C V_{y_{a} x_{b}} & C V_{y_{a} y_{b}} & C V_{y a x_{c}} & C V_{y_{a} y_{c}} \\
C V_{x_{b} x_{a}} & C V_{x_{b} y_{a}} & V_{x_{b} x_{b}} & C V_{x_{b} y_{b}} & C V_{x_{b} x_{c}} & C V_{x_{b} y_{c}} \\
C V_{y_{b} x_{a}} & C V_{y_{b} y_{a}} & C V_{y_{b} x_{b}} & V_{y_{b} y_{b}} & C V_{y_{b} x_{c}} & C V_{y_{b} x_{c}} \\
C V_{x_{c} x_{a}} & C V_{x_{c} y_{a}} & C V_{x_{c} x_{b}} & C V_{x_{c} y_{b}} & V_{x_{c} x_{c}} & C V_{x_{c} y_{c}} \\
C V_{y_{c} x_{a}} & C V_{y_{c} y_{a}} & C V_{y_{c} x_{b}} & C V_{y_{c} y_{b}} & C V_{y_{c} x_{c}} & V_{y_{c} y_{c}}
\end{array}\right)
$$

where $V$ is the variance among the same variable and $C V$ is the covariance among the different variables. Put simply, the diagonals are the variance measure $(V)$ and off-diagonals are the covariance values $(\mathrm{CV})$. The small off-diagonal values indicate the statistical independence among the variables. However, if these values come out as big numbers then this indicates redundancy in the system data, which can be eliminated through the diagonalization of the whole matrix. This means that all off-diagonal values will be reduced to zero and the critical information of the system will be restricted to the first and uppermost diagonal value (eigenvalue). In some cases, it may go up to the second and third eigenvalue, which are known as first, second, and third PCA scores of a system. Finally, to obtain the PCA results, one can always decompose the $C_{X}$ in the eigenvalues and vectors due to its symmetric nature, as shown in Equation (A4):

$$
C_{X}=X X^{T}=S \Omega S^{T}
$$

where $S$ is the matrix of eigenvectors and $\Omega$ is the eigenvalue diagonal matrix of the $V C_{X}$. The eigenvalue diagonal matrix $(\Omega)$ can be obtained by taking the dot product of the original dataset with the transpose of the eigenvector matrix of the $V C_{X}$, as shown in Equation (A5):

$$
\begin{gathered}
Y=X S^{T} \\
C_{Y}=\frac{1}{n-1} Y Y^{T}
\end{gathered}
$$

where $Y$ is matrix and $C_{Y}$ is a correlation matrix. After calculation and replacing the $Y=X S^{T}$ in Equation (A6), the final diagonal eigenvalue matrix can be obtained to determine the PCA scores of the system:

$$
C_{Y}=S^{T} S \Omega S^{T} S=\Omega
$$

\section{Appendix A.1. Chemiresistive Gas Sensors}

A chemiresistive gas sensor is a device that detects different levels of gas concentrations and shows the change in resistance at the output. The increase or decrease in the resistance depends on the nature of the sensing material and target gases (oxidizing/reducing). The oxidizing gases trap while the reducing gases donate the electrons during the reaction process at the sensing material surface.

\section{Appendix A.2. MOSFET Gas Sensors}

A MOSFET device consists of three terminals: drain (D), source (S), and gate (G). In MOSFETs, the drain to source current (IDS) flows through the thin layer of metal oxidesensing material. The device shows the change in IDS values after the adsorption of target gas molecules on the sensing material surface.

\section{Appendix A.3. Smart Gas Sensors}

A smart gas sensor is a device that efficiently and accurately detects particular gas traces among several other gases through a unique pattern recognition method using machine learning. 


\section{Appendix A.4. Breath Analyzers}

A breath analyzer is a non-invasive device that is used to precisely detect a particular gas or VOC at a specific concentration level among thousands of other VOCs in human breath for accurate disease diagnosis.

\section{Appendix A.5. Selectivity}

Selectivity can be defined as the ability of a sensor to accurately detect the traces of a particular gas among several other gases in the environment.

\section{Appendix A.6. Classifiers}

Classifiers are used to accurately discriminate among different classes. Firstly, unsupervised data processing techniques such as PCA and LDA are used to generate unique signatures for each class, and then a classifier is trained on this processed data to accurately predict different classes from an unseen dataset.

\section{Nomenclature}

MOSFET Metal-oxide-semiconductor field effect transistors

SPR Surface plasmon resonance

rGO Reduced graphene oxide

CNTs Carbon nanotubes

2D Two-dimensional

3D Three-dimensional

TMDCs Transition metal dichalcogenides

MOx Metal oxides

VOCs Volatile organic compounds

e-noses Electronic noses

FFT Fast Fourier transform

CWT Continuous wavelet transform

DWT Discrete wavelet transform

PCA Principal component analysis

LDA Linear discriminant analysis

KNN $k$-nearest neighbors

CART Classification and regression trees

NB Gaussian naïve Bayes

SVM Support vector machines

RF Random forest

DFT Density function theory

DOS Density of state

VSCs Volatile sulfur compounds

LOD Limit of detection

LPWN Low-power wireless network

M2M Machine to machine

LR Logistic regression

$R_{\text {air }} \quad$ Sensor resistance in air

$R_{\text {gas }} \quad$ Sensor resistance after gas exposure

$\Delta \mathrm{R} \quad$ Difference between $R_{\text {air }}$ and $R_{\text {gas }}$

$A_{\text {Resp }} \quad$ Area under response time curve

$A_{\text {Recov }} \quad$ Area under recovery time curve

Cavitand A container shaped molecule with a cavity

Tsfresh A tool for feature extraction

FETs Field effect transistors

CV Cross-validation

ANN Artificial neural networks

DFA Discriminant factor analysis

MEMS Micro-electromechanical systems

NEMS Nano-electromechanical systems 


\section{References}

1. Calderon-Garciduenas, L.; Azzarelli, B.; Acuna, H.; Garcia, R.; Gambling, T.M.; Osnaya, N.; Monroy, S.; Tizapantzi, M.D.; Carson, J.L.; Villarreal-Calderon, A.; et al. Air Pollution and Brain Damage. Toxicol. Pathol. 2002, 30, 373-389. [CrossRef] [PubMed]

2. Bernstein, J.A.; Alexis, N.; Barnes, C.; Bernstein, I.L.; Nel, A.; Peden, D.; Diaz-Sanchez, D.; Tarlo, S.M.; Williams, P.B. Health effects of air pollution. J. Allergy Clin. Immunol. 2004, 114, 1116-1123. [CrossRef]

3. Tsujita, W.; Yoshino, A.; Ishida, H.; Moriizumi, T. Gas sensor network for air-pollution monitoring. Sens. Actuators B Chem. 2005, 110, 304-311. [CrossRef]

4. Dmitriev, S.; Duca, G.; Dementiev, I.; Craciun, A. Flexible Substrate Based Gas Sensors for Air Pollution Monitoring. Mater. Res. Soc. Symp. Proc. 2004, 814, 169-174. [CrossRef]

5. Manisalidis, I.; Stavropoulou, E.; Stavropoulos, A.; Bezirtzoglou, E. Environmental and Health Impacts of Air Pollution: A Review. Front. Public Health 2020, 8, 14. [CrossRef] [PubMed]

6. Joshi, N.; Hayasaka, T.; Liu, Y.M.; Liu, H.L.; Oliveira, O.N.; Lin, L.W. A review on chemiresistive room temperature gas sensors based on metal oxide nanostructures, graphene and 2D transition metal dichalcogenides. Microchim. Acta 2018, $185,213$. [CrossRef]

7. Baron, R.; Saffell, J. Amperometric Gas Sensors as a Low Cost Emerging Technology Platform for Air Quality Monitoring Applications: A Review. ACS Sens. 2017, 2, 1553-1566. [CrossRef]

8. Liu, T.; Wang, X.N.; Li, L.; Yu, J.K. Review-Electrochemical NOxGas Sensors Based on Stabilized Zirconia. J. Electrochem. Soc. 2017, 164, B610-B619. [CrossRef]

9. Bondavalli, P.; Legagneux, P.; Pribat, D. Carbon nanotubes based transistors as gas sensors: State of the art and critical review. Sens. Actuators B Chem. 2009, 140, 304-318. [CrossRef]

10. Hubert, T.; Boon-Brett, L.; Black, G.; Banach, U. Hydrogen sensors-A review. Sens. Actuators B Chem. 2011, 157, 329-352. [CrossRef]

11. Joe, H.E.; Yun, H.; Jo, S.H.; Jun, M.B.G.; Min, B.K. A review on optical fiber sensors for environmental monitoring. Int. J. Precis. Eng. Manuf. Technol. 2018, 5, 173-191. [CrossRef]

12. Mujahid, A.; Dickert, F.L. Surface Acoustic Wave (SAW) for Chemical Sensing Applications of Recognition Layers. Sensors 2017, 17, 2716. [CrossRef]

13. Panneerselvam, G.; Thirumal, V.; Pandya, H.M. Review of Surface Acoustic Wave Sensors for the Detection and Identification of Toxic Environmental Gases/Vapours. Arch. Acoust. 2018, 43, 357-367.

14. Paschke, B.; Wixforth, A.; Denysenko, D.; Volkmer, D. Fast Surface Acoustic Wave-Based Sensors to Investigate the Kinetics of Gas Uptake in Ultra-Microporous Frameworks. ACS Sens. 2017, 2, 740-747. [CrossRef]

15. Singhal, A.V.; Charaya, H.; Lahiri, I. Noble Metal Decorated Graphene-Based Gas Sensors and Their Fabrication: A Review. Crit. Rev. Solid State 2017, 42, 499-526. [CrossRef]

16. Varghese, S.S.; Lonkar, S.; Singh, K.K.; Swaminathan, S.; Abdala, A. Recent advances in graphene based gas sensors. Sens. Actuators B Chem. 2015, 218, 160-183. [CrossRef]

17. Wang, T.; Huang, D.; Yang, Z.; Xu, S.S.; He, G.L.; Li, X.L.; Hu, N.T.; Yin, G.L.; He, D.N.; Zhang, L.Y. A Review on Graphene-Based Gas/Vapor Sensors with Unique Properties and Potential Applications. Nano-Micro Lett. 2016, 8, 95-119. [CrossRef] [PubMed]

18. Yaqoob, U.; Uddin, A.S.M.I.; Chung, G.S. Foldable hydrogen sensor using Pd nanocubes dispersed into multiwall carbon nanotubes-reduced graphene oxide network assembled on nylon filter membrane. Sens. Actuators B Chem. 2016, 229, 355-361. [CrossRef]

19. Kumar, S.; Pavelyev, V.; Mishra, P.; Tripathi, N. A review on chemiresistive gas sensors based on carbon nanotubes: Device and technology transformation. Sens. Actuators A Phys. 2018, 283, 174-186. [CrossRef]

20. Li, M.X.; Chen, T.; Gooding, J.J.; Liu, J.Q. Review of Carbon and Graphene Quantum Dots for Sensing. ACS Sens. 2019, 4, 1732-1748. [CrossRef]

21. Raeyani, D.; Shojaei, S.; Kandjani, S.A.; Wlodarski, W. Synthesizing Graphene Quantum Dots for Gas Sensing Applications. Procedia Eng. 2016, 168, 1312-1316. [CrossRef]

22. Wang, R.X.; Li, G.L.; Dong, Y.Q.; Chi, Y.W.; Chen, G.N. Carbon Quantum Dot-Functionalized Aerogels for $\mathrm{NO}_{2}$ Gas Sensing. Anal. Chem. 2013, 85, 8065-8069. [CrossRef]

23. Xia, Y.; Li, R.; Chen, R.S.; Wang, J.; Xiang, L. 3D Architectured Graphene/Metal Oxide Hybrids for Gas Sensors: A Review. Sensors 2018, 18, 1456. [CrossRef]

24. Chen, Z.; Wang, J.R.; Umar, A.; Wang, Y.; Li, H.; Zhou, G.F. Three-Dimensional Crumpled Graphene-Based Nanosheets with Ultrahigh NO2 Gas Sensibility. ACS Appl. Mater. Interfaces 2017, 9, 11819-11827. [CrossRef]

25. Kumar, R.; Goel, N.; Hojamberdiev, M.; Kumar, M. Transition metal dichalcogenides-based flexible gas sensors. Sens. Actuators A Phys. 2020, 303, 111875. [CrossRef]

26. Lee, E.; Yoon, Y.S.; Kim, D.J. Two-Dimensional Transition Metal Dichalcogenides and Metal Oxide Hybrids for Gas Sensing. ACS Sens. 2018, 3, 2045-2060. [CrossRef]

27. Zhu, L.; Zeng, W. Room-temperature gas sensing of ZnO-based gas sensor: A review. Sens. Actuators A Phys. 2017, 267, 242-261. [CrossRef] 
28. Das, S.; Jayaraman, V. SnO2: A comprehensive review on structures and gas sensors. Prog. Mater. Sci. 2014, 66, 112-255. [CrossRef]

29. Maziarz, W.; Kusior, A.; Trenczek-Zajac, A. Nanostructured TiO2-based gas sensors with enhanced sensitivity to reducing gases. Beilstein J. Nanotech. 2016, 7, 1718-1726. [CrossRef]

30. Dong, C.J.; Zhao, R.J.; Yao, L.J.; Ran, Y.; Zhang, X.; Wang, Y.D. A review on WO3 based gas sensors: Morphology control and enhanced sensing properties. J. Alloy. Compd. 2020, 820, 153194. [CrossRef]

31. Liu, W.; Xie, Y.L.; Chen, T.X.; Lu, Q.X.; Rehman, S.U.; Zhu, L. Rationally designed mesoporous In2O3 nanofibers functionalized Pt catalysts for high-performance acetone gas sensors. Sens. Actuators B Chem. 2019, 298, 126871. [CrossRef]

32. Wei, L.; Sun, J.; Xu, L.; Zhu, S.D.; Zhou, X.Y.; Yang, S.; Dong, B.; Bai, X.; Lu, G.Y.; Song, H.W. Understanding the noble metal modifying effect on In2O3 nanowires: Highly sensitive and selective gas sensors for potential early screening of multiple diseases. Nanoscale Horiz. 2019, 4, 1361-1371.

33. Mokoena, T.P.; Swart, H.C.; Motaung, D.E. A review on recent progress of p-type nickel oxide based gas sensors: Future perspectives. J. Alloy Compd. 2019, 805, 267-294. [CrossRef]

34. Mirzaei, A.; Janghorban, K.; Hashemi, B.; Bonyani, M.; Leonardi, S.G.; Neri, G. A novel gas sensor based on Ag/Fe2O3 core-shell nanocomposites. Ceram. Int. 2016, 42, 18974-18982. [CrossRef]

35. Mirzaei, A.; Hashemi, B.; Janghorban, K. $\alpha$-Fe2O3 based nanomaterials as gas sensors. J. Mater. Sci. Mater. Electron. 2016, 27, 3109-3144. [CrossRef]

36. Zhang, S.D.; Yang, M.J.; Liang, K.Y.; Turak, A.; Zhang, B.X.; Meng, D.; Wang, C.X.; Qu, F.D.; Cheng, W.L.; Yang, M.H. An acetone gas sensor based on nanosized Pt-loaded Fe2O3 nanocubes. Sens. Actuators B Chem. 2019, 290, 59-67. [CrossRef]

37. Fine, G.F.; Cavanagh, L.M.; Afonja, A.; Binions, R. Metal Oxide Semi-Conductor Gas Sensors in Environmental Monitoring. Sensors 2010, 10, 5469-5502. [CrossRef]

38. Choi, S.J.; Kim, I.D. Recent Developments in 2D Nanomaterials for Chemiresistive-Type Gas Sensors. Electron. Mater. Lett. 2018, 14, 221-260. [CrossRef]

39. Wu, J.; Wu, Z.X.; Ding, H.J.; Yang, X.; Wei, Y.M.; Xiao, M.Q.; Yang, Z.Q.; Yang, B.R.; Liu, C.; Lu, X.; et al. Three-DimensionalStructured Boron- and Nitrogen-Doped Graphene Hydrogel Enabling High-Sensitivity NO2 Detection at Room Temperature. ACS Sens. 2019, 4, 1889-1898. [CrossRef] [PubMed]

40. Rahaman, M.H.; Yaqoob, U.; Kim, H.C. Fast Hydrogenation and Dehydrogenation of Pt/Pd Bimetal Decorated over NanoStructured Ag Islands Grown on Alumina Substrates. Sensors 2019, 19, 86. [CrossRef]

41. Rahaman, M.H.; Yaqoob, U.; Kim, H.C. Fast hydrogenation and dehydrogenation of Pd-Mg bimetal capped Ti nano-particles layer deposited on Si substrate. Sens. Actuators B Chem. 2020, 309, 127814. [CrossRef]

42. Yaqoob, U.; Uddin, A.I.; Chung, G.S. A high-performance flexible NO2 sensor based on WO3 NPs decorated on MWCNTs and RGO hybrids on PI/PET substrates. Sens. Actuators B Chem. 2016, 224, 738-746. [CrossRef]

43. Yaqoob, U.; Chung, G.S. Highly flexible room temperature NO2 sensor based on WO3 nanoparticles loaded MWCNTs-RGO hybrid. Proc. SPIE 2016, 9749. [CrossRef]

44. Ji, H.C.; Zeng, W.; Li, Y.Q. Gas sensing mechanisms of metal oxide semiconductors: A focus review. Nanoscale 2019, 11, 22664-22684. [CrossRef]

45. Al-Hashem, M.; Akbar, S.; Morris, P. Role of Oxygen Vacancies in Nanostructured Metal-Oxide Gas Sensors: A Review. Sens. Actuators B Chem. 2019, 301. [CrossRef]

46. Xu, Y.; Zheng, L.; Yang, C.; Zheng, W.; Liu, X.; Zhang, J. Oxygen Vacancies Enabled Porous SnO2 Thin Films for Highly Sensitive Detection of Triethylamine at Room Temperature. ACS Appl. Mater. Interfaces 2020, 12, 20704-20713. [CrossRef] [PubMed]

47. Wang, Z.Y.; Zhao, C.; Han, T.Y.; Zhang, Y.; Liu, S.; Fei, T.; Lu, G.Y.; Zhang, T. High-performance reduced graphene oxide-based room-temperature NO2 sensors: A combined surface modification of $\mathrm{SnO} 2$ nanoparticles and nitrogen doping approach. Sens. Actuators B Chem. 2017, 242, 269-279. [CrossRef]

48. Suematsu, K.; Watanabe, K.; Tou, A.; Sun, Y.J.; Shimanoe, K. Ultraselective Toluene-Gas Sensor: Nanosized Gold Loaded on Zinc Oxide Nanoparticles. Anal. Chem. 2018, 90, 1959-1966. [CrossRef] [PubMed]

49. Ueda, T.; Boehme, I.; Hyodo, T.; Shimizu, Y.; Weimar, U.; Barsan, N. Enhanced NO2-Sensing Properties of Au-Loaded Porous In2O3 Gas Sensors at Low Operating Temperatures. Chemosensors 2020, 8, 72. [CrossRef]

50. Zappa, D.; Galstyan, V.; Kaur, N.; Arachchige, H.M.M.M.; Sisman, O.; Comini, E. Metal oxide -based heterostructures for gas sensors-A review. Anal. Chim. Acta 2018, 1039, 1-23. [CrossRef] [PubMed]

51. Walker, J.M.; Akbar, S.A.; Morris, P.A. Synergistic effects in gas sensing semiconducting oxide nano-heterostructures: A review. Sens. Actuators B Chem. 2019, 286, 624-640. [CrossRef]

52. Karnati, P.; Akbar, S.; Morris, P.A. Conduction mechanisms in one dimensional core-shell nanostructures for gas sensing: A review. Sens. Actuators B Chem. 2019, 295, 127-143. [CrossRef]

53. Majhi, S.M.; Rai, P.; Yu, Y.T. Facile Approach to Synthesize Au@ZnO Core-Shell Nanoparticles and Their Application for Highly Sensitive and Selective Gas Sensors. ACS Appl. Mater. Interfaces 2015, 7, 9462-9468. [CrossRef] [PubMed]

54. Tharsika, T.; Haseeb, A.S.M.A.; Akbar, S.A.; Sabri, M.F.M.; Hoong, W.Y. Enhanced Ethanol Gas Sensing Properties of SnO2Core/ZnO-Shell Nanostructures. Sensors 2014, 14, 14586-14600. [CrossRef] [PubMed]

55. Li, X.; Zhao, Y.; Wang, X.; Wang, J.; Gaskov, A.M.; Akbar, S. Reduced graphene oxide (rGO) decorated TiO2 microspheres for selective room-temperature gas sensors. Sens. Actuators B Chem. 2016, 230, 330-336. [CrossRef] 
56. Sanger, A.K.; Jeong, M.H.; Kim, C.U.; Baik, J.M.; Choi, K.J. All-Transparent NO2 Gas Sensors Based on Free-standing Al-Doped ZnO Nanofibers. ACS Appl. Electron. Mater. 2019, 1, 1261-1268. [CrossRef]

57. Li, G.; Cheng, Z.; Xiang, Q.; Yan, L.; Wang, X.; Xu, J. Bimetal PdAu decorated SnO2 nanosheets based gas sensor with temperaturedependent dual selectivity for detecting formaldehyde and acetone. Sens. Actuators B Chem. 2019, 283, 590-601. [CrossRef]

58. Xu, D.X.; Wang, X.; Chen, Y.; Yu, H.; Zheng, D.; Li, X. Pentagram-Shaped Ag@Pt Core-Shell Nanostructures as High-Performance Catalysts for Formaldehyde Detection. ACS Appl. Mater. Interfaces 2020, 12, 8091-8097. [CrossRef]

59. Feng, S.; Farha, F.; Li, Q.; Wan, Y.; Xu, Y.; Zhang, T.; Ning, H. Review on Smart Gas Sensing Technology. Sensors 2019, 19, 3760. [CrossRef]

60. Hu, W.; Wan, L.; Jian, Y.; Ren, C.; Jin, K.; Su, X.; Bai, X.; Haick, H.; Yao, M.; Wu, W. Electronic Noses: From Advanced Materials to Sensors Aided with Data Processing. Adv. Mater. Technol. 2019, 4, 2. [CrossRef]

61. Röck, F.; Barsan, N.; Weimar, U. Electronic Nose: Current Status and Future Trends. Chem. Rev. 2008, 108, 705-725. [CrossRef]

62. Liu, Q.; Li, X.; Ye, M.; Ge, S.S.; Du, X. Drift Compensation for Electronic Nose by Semi-Supervised Domain Adaption. IEEE Sens. J. 2014, 14, 657-665. [CrossRef]

63. Wenzel, M.J.; Mensah-Brown, A.; Josse, F.; Yaz, E.E. Online Drift Compensation for Chemical Sensors Using Estimation Theory. IEEE Sens. J. 2011, 11, 225-232. [CrossRef]

64. Padilla, M.; Perera, A.; Montoliu, I.; Chaudry, A.; Persaud, K.; Marco, S. Drift compensation of gas sensor array data by Orthogonal Signal Correction. Chemom. Intell. Lab. Syst. 2010, 100, 28-35. [CrossRef]

65. Ziyatdinov, A.; Marco, S.; Chaudry, A.; Persaud, K.; Caminal, P.; Perera, A. Drift compensation of gas sensor array data by common principal component analysis. Sens. Actuators B Chem. 2010, 146, 460-465. [CrossRef]

66. Gutierrez-Osuna, R. Pattern analysis for machine olfaction: A review. IEEE Sens. J. 2002, 2, 189-202. [CrossRef]

67. Pan, S.J.; Tsang, I.W.; Kwok, J.T.; Yang, Q. Domain Adaptation via Transfer Component Analysis. IEEE Trans. Neural Networks 2011, 22, 199-210. [CrossRef]

68. Xue, B.; Zhang, M.; Browne, W.N.; Yao, X. A Survey on Evolutionary Computation Approaches to Feature Selection. IEEE Trans. Evol. Comput. 2016, 20, 606-626. [CrossRef]

69. Distante, C.; Leo, M.; Siciliano, P.; Persaud, K.C. On the study of feature extraction methods for an electronic nose. Sens. Actuators B Chem. 2002, 87, 274-288. [CrossRef]

70. Yan, J.; Guo, X.Z.; Duan, S.K.; Jia, P.F.; Wang, L.D.; Peng, C.; Zhang, S.L. Electronic Nose Feature Extraction Methods: A Review. Sensors 2015, 15, 27804-27831. [CrossRef]

71. Yan, M.; Tylczak, J.; Yu, Y.; Panagakos, G.; Ohodnicki, P. Multi-component optical sensing of high temperature gas streams using functional oxide integrated silica based optical fiber sensors. Sens. Actuators B Chem. 2018, 255, 357-365. [CrossRef]

72. Casey, J.G.; Collier-Oxandale, A.; Hannigan, M. Performance of artificial neural networks and linear models to quantify 4 trace gas species in an oil and gas production region with low-cost sensors. Sens. Actuators B Chem. 2019, 283, 504-514. [CrossRef]

73. Salhi, L.; Silverston, T.; Yamazaki, T.; Miyoshi, T. Early Detection System for Gas Leakage and Fire in Smart Home Using Machine Learning. In Proceedings of the 2019 IEEE International Conference on Consumer Electronics (ICCE), Las Vegas, NV, USA, 11-13 January 2019.

74. Miller, D.R.; Williams, R.E.; Akbar, S.A.; Morris, P.A.; McComb, D.W. STEM-Cathodoluminescence of SnO2 nanowires and powders. Sens. Actuators B Chem. 2017, 240, 193-203. [CrossRef]

75. Zhuang, Y.; Liu, L.; Wu, X.; Tian, Y.; Zhou, X.; Xu, S.; Xie, Z.; Ma, Y. Size and Shape Effect of Gold Nanoparticles in "Far-Field" Surface Plasmon Resonance. Part. Part. Syst. Charact. 2019, 36, 1. [CrossRef]

76. Filippo, E.; Manno, D.; Buccolieri, A.; Di Giulio, M.; Serra, A. Shape-dependent plasmon resonances of Ag nanostructures. Superlattice Microst. 2010, 47, 66-71. [CrossRef]

77. Nah, J.; Kumar, S.B.; Fang, H.; Chen, Y.-Z.; Plis, E.; Chueh, Y.-L.; Krishna, S.; Guo, J.; Javey, A. Quantum Size Effects on the Chemical Sensing Performance of Two-Dimensional Semiconductors. J. Phys. Chem. C 2012, 116, 9750-9754. [CrossRef]

78. Katoch, A.; Sun, G.-J.; Choi, S.-W.; Byun, J.-H.; Kim, S.S. Competitive influence of grain size and crystallinity on gas sensing performances of $\mathrm{ZnO}$ nanofibers. Sens. Actuators B Chem. 2013, 185, 411-416. [CrossRef]

79. Miller, D.R.; Akbar, S.A.; Morris, P.A. Nanoscale metal oxide-based heterojunctions for gas sensing: A review. Sens. Actuators $B$ Chem. 2014, 204, 250-272. [CrossRef]

80. Qian, G.; Peng, Q.; Zou, D.; Wang, S.; Yan, B.; Zhou, Q. First-Principles Insight Into Au-Doped MoS2 for Sensing C2H6 and C2H4. Front. Mater. 2020, 7, 7. [CrossRef]

81. Phan, D.T.; Youn, J.S.; Jeon, K.J. High-sensitivity and fast-response hydrogen sensor for safety application using Pt nanoparticledecorated 3D graphene. Renew. Energy 2019, 144, 167-171. [CrossRef]

82. Ewers, T.D.; Sra, A.K.; Schaak, R.E. Spontaneous hierarchical assembly of rhodium nanoparticles into spherical aggregates and superlattices. Abstr. Pap. Am. Chem. S 2005, 229, U522. [CrossRef]

83. Wiley, B.; Sun, Y.G.; Xia, Y.N. Synthesis of silver nanostructures with controlled shapes and properties. Acc. Chem. Res. 2007, 40, 1067-1076. [CrossRef] [PubMed]

84. Rai, P.; Raj, S.; Ko, K.-J.; Park, K.-K.; Yu, Y.-T. Synthesis of flower-like ZnO microstructures for gas sensor applications. Sens. Actuators B Chem. 2013, 178, 107-112. [CrossRef]

85. Chang, C.-J.; Lin, C.-Y.; Chen, J.-K.; Hsu, M.-H. Ce-doped ZnO nanorods based low operation temperature NO2 gas sensors. Ceram. Int. 2014, 40, 10867-10875. [CrossRef] 
86. Yu, L.M.; Guo, F.; Liu, S.; Yang, B.; Jiang, Y.X.; Qi, L.J.; Fan, X.H. Both oxygen vacancies defects and porosity facilitated NO2 gas sensing response in 2D ZnO nanowalls at room temperature. J. Alloy Compd. 2016, 682, 352-356. [CrossRef]

87. Feng, Y.; Gong, S.; Du, E.; Chen, X.; Qi, R.; Yu, K.; Zhu, Z.-Q. 3R TaS2 Surpasses the Corresponding $1 \mathrm{~T}$ and 2H Phases for the Hydrogen Evolution Reaction. J. Phys. Chem. C 2018, 122, 2382-2390. [CrossRef]

88. Xia, C.; Zhao, X.; Peng, Y.; Zhang, H.; Wei, S.; Jia, Y. First-principles study of group V and VII impurities in SnS2. Superlattices Microstruct. 2015, 85, 664-671. [CrossRef]

89. Phan, D.-T.; Chung, G.-S. A novel Pd nanocube-graphene hybrid for hydrogen detection. Sens. Actuators B Chem. 2014, 199, 354-360. [CrossRef]

90. Zhang, L.; Zhang, F.; Yang, X.; Long, G.K.; Wu, Y.P.; Zhang, T.F.; Leng, K.; Huang, Y.; Ma, Y.F.; Yu, A.; et al. Porous 3D graphene-based bulk materials with exceptional high surface area and excellent conductivity for supercapacitors. Sci. Rep. 2013, 3, 1-9. [CrossRef]

91. Wan, X.; Huang, Y.; Chen, Y. Focusing on Energy and Optoelectronic Applications: A Journey for Graphene and Graphene Oxide at Large Scale. Acc. Chem. Res. 2011, 45, 598-607. [CrossRef]

92. Burman, D.; Santra, S.; Pramanik, P.; Guha, P.K. Pt decorated MoS2nanoflakes for ultrasensitive resistive humidity sensor. Nanotechnology 2018, 29, 115504. [CrossRef] [PubMed]

93. Basharnavaz, H.; Habibi-Yangjeh, A.; Kamali, S.H. A first-principle investigation of NO2 adsorption behavior on Co, Rh, and Ir-embedded graphitic carbon nitride: Looking for highly sensitive gas sensor. Phys. Lett. A 2020, 384, 126057. [CrossRef]

94. Cui, S.; Pu, H.; Wells, S.A.; Wen, Z.; Mao, S.; Chang, J.; Hersam, M.C.; Chen, J. Ultrahigh sensitivity and layer-dependent sensing performance of phosphorene-based gas sensors. Nat. Commun. 2015, 6, 8632. [CrossRef] [PubMed]

95. Varghese, S.S.S.; Sing, K.K.; Mittal, V. Ab initio study on gas sensing properties of group III (B, Al and Ga) doped graphene. Comput. Condens. Matter 2016, 9, 40-55. [CrossRef]

96. Wang, W.; Zhang, Y.; Shen, C.; Chai, Y. Adsorption of CO molecules on doped graphene: A first-principles study. AIP Adv. 2016, 6, 025317. [CrossRef]

97. Tang, Y.; Liu, Z.; Shen, Z.; Chen, W.; Ma, D.; Dai, X. Adsorption sensitivity of metal atom decorated bilayer graphene toward toxic gas molecules (CO, NO, SO2 and HCN). Sens. Actuators B Chem. 2017, 238, 182-195. [CrossRef]

98. Wang, J.X.; Zhou, Q.; Lu, Z.R.; Gui, Y.G.; Zeng, W. Adsorption of H2O molecule on TM (Au, Ag) doped-MoS2 mono-layer: A first-principles study. Phys. E 2019, 113, 72-78. [CrossRef]

99. Yue, Q.; Shao, Z.; Chang, S.; Li, J. Adsorption of gas molecules on monolayer MoS2 and effect of applied electric field. Nanoscale Res. Lett. 2013, 8, 425. [CrossRef]

100. Saadi, L.; Lambert-Mauriat, C.; Oison, V.; Ouali, H.; Hayn, R. Mechanism of NOx sensing on WO3 surface: First principle calculations. Appl. Surf. Sci. 2014, 293, 76-79. [CrossRef]

101. Bai, S.; Guo, T.; Zhao, Y.; Luo, R.; Li, D.; Chen, A.; Liu, C.C. Mechanism enhancing gas sensing and first-principle calculations of Al-doped ZnO nanostructures. J. Mater. Chem. A 2013, 1, 11335-11342. [CrossRef]

102. Kang, I.-S.; So, H.-M.; Bang, G.-S.; Kwak, J.-H.; Lee, J.-O.; Ahn, C.W. Recovery improvement of graphene-based gas sensors functionalized with nanoscale heterojunctions. Appl. Phys. Lett. 2012, 101, 123504. [CrossRef]

103. Huang, L.; Wang, Z.P.; Zhang, J.K.; Pu, J.L.; Lin, Y.J.; Xu, S.H.; Shen, L.; Chen, Q.; Shi, W.Z. Fully Printed, Rapid-Response Sensors Based on Chemically Modified Graphene for Detecting NO2 at Room Temperature. ACS Appl. Mater. Interfaces 2014, 6, 7426-7433. [CrossRef]

104. Kim, Y.; Choi, Y.S.; Park, S.Y.; Kim, T.; Hong, S.-P.; Lee, T.H.; Moon, C.W.; Lee, J.-H.; Lee, D.; Hong, B.H.; et al. Au decoration of a graphene microchannel for self-activated chemoresistive flexible gas sensors with substantially enhanced response to hydrogen. Nanoscale 2019, 11, 2966-2973. [CrossRef] [PubMed]

105. Jang, J.-S.; Lee, J.; Koo, W.-T.; Kim, D.-H.; Cho, H.-J.; Shin, H.; Kim, I.-D. Pore-Size-Tuned Graphene Oxide Membrane as a Selective Molecular Sieving Layer: Toward Ultraselective Chemiresistors. Anal. Chem. 2020, 92, 957-965. [CrossRef]

106. Late, D.J.; Huang, Y.-K.; Liu, B.; Acharya, J.; Shirodkar, S.N.; Luo, J.; Yan, A.; Charles, D.; Waghmare, U.V.; Dravid, V.P.; et al. Sensing Behavior of Atomically Thin-Layered MoS2 Transistors. ACS Nano 2013, 7, 4879-4891. [CrossRef]

107. Park, J.; Mun, J.H.; Shin, J.S.; Kang, S.W. Highly sensitive two-dimensional MoS2 gas sensor decorated with Pt nanoparticles. R. Soc. Open Sci. 2018, 5, 181462. [CrossRef] [PubMed]

108. Dey, A. Semiconductor metal oxide gas sensors: A review. Mater. Sci. Eng. B Adv. 2018, 229, 206-217. [CrossRef]

109. Kolmakov, A.; Klenov, D.O.; Lilach, Y.; Stemmer, S.; Moskovits, M. Enhanced gas sensing by individual SnO2 nanowires and nanobelts functionalized with Pd catalyst particles. Nano Lett. 2005, 5, 667-673. [CrossRef]

110. Chung, F.C.; Zhu, Z.; Luo, P.Y.; Wu, R.J.; Li, W. Au@ZnO core-shell structure for gaseous formaldehyde sensing at room temperature. Sens. Actuators B Chem. 2014, 199, 314-319. [CrossRef]

111. Zhu, L.Y.; Yuan, K.P.; Yang, J.G.; Ma, H.P.; Wang, T.; Ji, X.M.; Feng, J.J.; Devi, A.; Lu, H.L. Fabrication of hetero-structured $\mathrm{p}-\mathrm{CuO} / \mathrm{n}-\mathrm{SnO} 2$ core-shell nanowires for enhanced sensitive and selective formaldehyde detection. Sens. Actuators B Chem. 2019, 290, 233-241. [CrossRef]

112. Horprathum, M.; Srichaiyaperk, T.; Samransuksamer, B.; Wisitsoraat, A.; Eiamchai, P.; Limwichean, S.; Chananonnawathorn, C.; Aiempanakit, K.; Nuntawong, N.; Patthanasettakul, V.; et al. Ultrasensitive Hydrogen Sensor Based on Pt-Decorated WO3 Nanorods Prepared by Glancing-Angle dc Magnetron Sputtering. ACS Appl. Mater. Interfaces 2014, 6, 22051-22060. [CrossRef] 
113. Mattoni, G.; De Jong, B.; Manca, N.; Tomellini, M.; Caviglia, A.D. Single-Crystal Pt-Decorated WO3 Ultrathin Films: A Platform for Sub-ppm Hydrogen Sensing at Room Temperature. ACS Appl. Nano Mater. 2018, 1, 3446-3452. [CrossRef] [PubMed]

114. Choi, S.-J.; Lee, I.; Jang, B.-H.; Youn, D.-Y.; Ryu, W.-H.; Park, C.O.; Kim, I.-D. Selective Diagnosis of Diabetes Using PtFunctionalized WO3Hemitube Networks as a Sensing Layer of Acetone in Exhaled Breath. Anal. Chem. 2013, 85, 1792-1796. [CrossRef]

115. Penza, M.; Martucci, C.; Cassano, G. NOx gas sensing characteristics of WO3 thin films activated by noble metals (Pd, Pt, Au) layers. Sens. Actuators B Chem. 1998, 50, 52-59. [CrossRef]

116. Stankova, A.; Vilanova, X.; Calderer, J.; Llobet, E.; Ivanov, P.; Gracia, I.; Cane, C.; Correig, X. Detection of SO2 and (HS)-S-2 in CO2 stream by means of WO3-based micro-hotplate sensors. Sens. Actuators B Chem. 2004, 102, 219-225. [CrossRef]

117. Shingange, K.; Tshabalala, Z.; Ntwaeaborwa, O.; Motaung, D.; Mhlongo, G. Highly selective NH3 gas sensor based on Au loaded $\mathrm{ZnO}$ nanostructures prepared using microwave-assisted method. J. Colloid Interface Sci. 2016, 479, 127-138. [CrossRef] [PubMed]

118. Arunkumar, S.; Hou, T.; Kim, Y.-B.; Choi, B.; Park, S.H.; Jung, S.; Lee, D.-W. Au Decorated ZnO hierarchical architectures: Facile synthesis, tunable morphology and enhanced CO detection at room temperature. Sens. Actuators B Chem. 2017, 243, 990-1001. [CrossRef]

119. Rambu, A.; Ursu, L.; Iftimie, N.; Nica, V.; Dobromir, M.; Iacomi, F. Study on Ni-doped ZnO films as gas sensors. Appl. Surf. Sci. 2013, 280, 598-604. [CrossRef]

120. Bai, Z.; Xie, C.; Hu, M.; Zhang, S. Formaldehyde sensor based on Ni-doped tetrapod-shaped ZnO nanopowder induced by external magnetic field. Phys. E 2008, 41, 235-239. [CrossRef]

121. Namuduri, S.; Narayanan, B.N.; Davuluru, V.S.P.; Burton, L.; Bhansali, S. Review—Deep Learning Methods for Sensor Based Predictive Maintenance and Future Perspectives for Electrochemical Sensors. J. Electrochem. Soc. 2020, 167, 037552. [CrossRef]

122. Guney, S.; Atasoy, A. Multiclass classification of n-butanol concentrations with k-nearest neighbor algorithm and support vector machine in an electronic nose. Sens. Actuators B Chem. 2012, 166, 721-725. [CrossRef]

123. Nallon, E.C.; Schnee, V.P.; Bright, C.J.; Polcha, M.P.; Li, Q. Chemical Discrimination with an Unmodified Graphene Chemical Sensor. ACS Sens. 2016, 1, 26-31. [CrossRef]

124. Tonezzer, M.; Le, D.T.T.; Iannotta, S.; Van Hieu, N. Selective discrimination of hazardous gases using one single metal oxide resistive sensor. Sens. Actuators B Chem. 2018, 277, 121-128. [CrossRef]

125. Itoh, T.; Koyama, Y.; Shin, W.; Akamatsu, T.; Tsuruta, A.; Masuda, Y.; Uchiyama, K. Selective Detection of Target Volatile Organic Compounds in Contaminated Air Using Sensor Array with Machine Learning: Aging Notes and Mold Smells in Simulated Automobile Interior Contaminant Gases. Sensors 2020, 20, 2687. [CrossRef] [PubMed]

126. Acharyya, S.; Jana, B.; Nag, S.; Saha, G.; Guha, P.K. Single resistive sensor for selective detection of multiple VOCs employing $\mathrm{SnO} 2$ hollowspheres and machine learning algorithm: A proof of concept. Sens. Actuators B Chem. 2020, 321, 128484. [CrossRef]

127. Llobet, E.; Ionescu, R.; Al-Khalifa, S.; Brezmes, J.; Vilanova, X.; Correig, X.; Barsan, N.; Gardner, J. Multicomponent gas mixture analysis using a single tin oxide sensor and dynamic pattern recognition. IEEE Sens. J. 2001, 1, 207-213. [CrossRef]

128. Kraus, G.; Weimar, U.; Gauglitz, G.; Gopel, W. Pattern-Recognition and Multicomponent Analysis in Chemical Sensing. Technol. Mess. 1995, 62, 229-236.

129. Shekhirev, M.; Lipatov, A.; Torres, A.; Vorobeva, N.S.; Harkleroad, A.; Lashkov, A.; Sysoev, V.; Sinitskii, A. Highly Selective Gas Sensors Based on Graphene Nanoribbons Grown by Chemical Vapor Deposition. ACS Appl. Mater. Interfaces 2020, 12, 7392-7402. [CrossRef]

130. Akamatsu, T.I.T.; Tsuruta, A.; Shin, W.; Itoh, T.; Akamatsu, T. Selective Detection of Target Volatile Organic Compounds in Contaminated Humid Air Using a Sensor Array with Principal Component Analysis. Sensors 2017, 17, 1662. [CrossRef] [PubMed]

131. Faleh, R.; Othman, M.; Kachouri, A.; Aguir, K.; Othman, M. Recognition of O3 concentration using WO3 gas sensor and principal component analysis. In Proceedings of the 2014 1st International Conference on Advanced Technologies for Signal and Image Processing (ATSIP), Sousse, Tunisia, 17-19 March 2014; pp. 322-326.

132. Jaeschke, C.; Glöckler, J.; El Azizi, O.; Gonzalez, O.; Padilla, M.; Mitrovics, J.; Mizaikoff, B. An Innovative Modular eNose System Based on a Unique Combination of Analog and Digital Metal Oxide Sensors. ACS Sens. 2019, 4, 2277-2281. [CrossRef]

133. Tonezzer, M.; Kim, J.H.; Lee, J.H.; Iannotta, S.; Kim, S.S. Predictive gas sensor based on thermal fingerprints from Pt-SnO2 nanowires. Sens. Actuators B Chem. 2019, 281, 670-678. [CrossRef]

134. Tonezzer, M. Selective gas sensor based on one single SnO2 nanowire. Sens. Actuators B Chem. 2019, 288, 53-59. [CrossRef]

135. Schroeder, V.; Evans, E.D.; Wu, Y.-C.M.; Voll, C.-C.A.; McDonald, B.R.; Savagatrup, S.; Swager, T.M. Chemiresistive Sensor Array and Machine Learning Classification of Food. ACS Sens. 2019, 4, 2101-2108. [CrossRef]

136. Feng, P.; Shao, F.; Shi, Y.; Wan, Q. Gas Sensors Based on Semiconducting Nanowire Field-Effect Transistors. Sensors 2014, 14, 17406-17429. [CrossRef]

137. Wang, B.; Cancilla, J.C.; Torrecilla, J.S.; Haick, H. Artificial Sensing Intelligence with Silicon Nanowires for Ultraselective Detection in the Gas Phase. Nano Lett. 2014, 14, 933-938. [CrossRef]

138. Guo, S.Y.; Li, B.; Dong, Q.; Li, Z.; Zaghloul, M.E. An Artificial Intelligent Flexible Gas Sensor Based on Ultra-Large Area MoSe2 Nanosheet. In Proceedings of the IEEE 62nd International Midwest Symposium on Circuits and Systems (MWSCAS), Dallas, TX, USA, 4-7 August 2019; pp. 884-887. 
139. Bian, L.; Sorescu, D.C.; Chen, L.; White, D.L.; Burkert, S.C.; Khalifa, Y.; Zhang, Z.W.; Sejdic, E.; Star, A. Machine-Learning Identification of the Sensing Descriptors Relevant in Molecular Interactions with Metal Nanoparticle-Decorated Nanotube Field-Effect Transistors. ACS Appl. Mater. Interfaces 2019, 11, 1219-1227. [CrossRef]

140. Hayasaka, T.; Lin, A.; Copa, V.C.; Lopez, L.P.; Loberternos, R.A.; Ballesteros, L.I.M.; Kubota, Y.; Liu, Y.; Salvador, A.A.; Lin, L. An electronic nose using a single graphene FET and machine learning for water, methanol, and ethanol. Microsyst. Nanoeng. 2020, 6 , 1-13. [CrossRef]

141. Shehada, N.; Bronstrup, G.; Funka, K.; Christiansen, S.; Leja, M.; Haick, H. Ultrasensitive Silicon Nanowire for Real-World Gas Sensing: Noninvasive Diagnosis of Cancer from Breath Volatolome. Nano Lett. 2015, 15, 1288-1295. [CrossRef] [PubMed]

142. Hsu, W.-E.; Chang, Y.-H.; Lin, C.-T. A Machine-Learning Assisted Sensor for Chemo-Physical Dual Sensing Based on Ion-Sensitive Field-Effect Transistor Architecture. IEEE Sens. J. 2019, 19, 9983-9990. [CrossRef]

143. Di Natale, C.; Paolesse, R.; Martinelli, E.; Capuano, R. Solid-state gas sensors for breath analysis: A review. Anal. Chim. Acta 2014, 824, 1-17. [CrossRef]

144. Ibañez, F.J.; Zamborini, F.P. Chemiresistive Sensing of Volatile Organic Compounds with Films of Surfactant-Stabilized Gold and Gold-Silver Alloy Nanoparticles. ACS Nano 2008, 2, 1543-1552. [CrossRef] [PubMed]

145. Jalal, A.H.; Alam, F.; Roychoudhury, S.; Umasankar, Y.; Pala, N.; Bhansali, S. Prospects and Challenges of Volatile Organic Compound Sensors in Human Healthcare. ACS Sens. 2018, 3, 1246-1263. [CrossRef] [PubMed]

146. Peng, G.; Tisch, U.; Adams, O.; Hakim, M.; Shehada, N.; Broza, Y.Y.; Billan, S.; Abdah-Bortnyak, R.; Kuten, A.; Haick, H. Diagnosing lung cancer in exhaled breath using gold nanoparticles. Nat. Nanotechnol. 2009, 4, 669-673. [CrossRef] [PubMed]

147. Peng, G.; Hakim, M.; Broza, Y.Y.; Billan, S.; Abdah-Bortnyak, R.; Kuten, A.; Tisch, U.; Haick, H. Detection of lung, breast, colorectal, and prostate cancers from exhaled breath using a single array of nanosensors. Br. J. Cancer 2010, 103, 542-551. [CrossRef]

148. Kahn, N.; Lavie, O.; Paz, M.; Segev, Y.; Haick, H. Dynamic Nanoparticle-Based Flexible Sensors: Diagnosis of Ovarian Carcinoma from Exhaled Breath. Nano Lett. 2015, 15, 7023-7028. [CrossRef]

149. Park, C.H.; Schroeder, V.; Kim, B.J.; Swager, T.M. Ionic Liquid-Carbon Nanotube Sensor Arrays for Human Breath Related Volatile Organic Compounds. ACS Sens. 2018, 3, 2432-2437. [CrossRef]

150. Sujono, H.A.R.; Amin, M. Asthma Identification Using Gas Sensors and Support Vector Machine. Telkomnika 2018, 6, 1468-1480. [CrossRef] 